\title{
The Kato Square Root Problem for Divergence Form Operators with Potential
}

\author{
Julian Bailey ${ }^{1}$ \\ Received: 7 October 2019 / Revised: 22 March 2020 / Published online: 12 June 2020 \\ (c) The Author(s) 2020
}

\section{Abstract}

The Kato square root problem for divergence form elliptic operators with potential $V: \mathbb{R}^{n} \rightarrow \mathbb{C}$ is the equivalence statement $\left\|(L+V)^{\frac{1}{2}} u\right\|_{2} \simeq\|\nabla u\|_{2}+\left\|V^{\frac{1}{2}} u\right\|_{2}$, where $L+V:=-\operatorname{div}(A \nabla)+V$ and the perturbation $A$ is an $L^{\infty}$ complex matrixvalued function satisfying an accretivity condition. This relation is proved for any potential with range contained in some positive sector and satisfying $\left\||V|^{\frac{\alpha}{2}} u\right\|_{2}+$ $\left\|(-\Delta)^{\frac{\alpha}{2}} u\right\|_{2} \lesssim\left\|(|V|-\Delta)^{\frac{\alpha}{2}} u\right\|_{2}$ for all $u \in D(|V|-\Delta)$ and some $\alpha \in(1,2]$. The class of potentials that will satisfy such a condition is known to contain the reverse Hölder class $\mathrm{RH}_{2}$ and $L^{\frac{n}{2}}\left(\mathbb{R}^{n}\right)$ in dimension $n>4$. To prove the Kato estimate with potential, a non-homogeneous version of the framework introduced by Axelsson, Keith and McIntosh for proving quadratic estimates is developed. In addition to applying this non-homogeneous framework to the scalar Kato problem with zero-order potential, it will also be applied to the Kato problem for systems of equations with zero-order potential.

Keywords Kato problem · Non-homogeneous · Schrödinger operator · Divergence form operator · Potential · Quadratic estimates

Mathematics Subject Classification Primary 42B37 · Secondary 35J10

Communicated by Fabio Nicola.

This research was partially supported by the Australian Research Council through the Discovery Project DP160100941.

\footnotetext{
$凶$ Julian Bailey

julianbailey230@gmail.com

1 School of Mathematics, The University of Birmingham, Birmingham B15 2TT, UK
} 


\section{Introduction}

For Hilbert spaces $\mathcal{H}$ and $\mathcal{K}$, let $\mathcal{L}(\mathcal{H}, \mathcal{K})$ denote the space of bounded linear operators from $\mathcal{H}$ to $\mathcal{K}$ and set $\mathcal{L}(\mathcal{H}):=\mathcal{L}(\mathcal{H}, \mathcal{H})$. Fix $n \in \mathbb{N}^{*}=\mathbb{N} \backslash\{0\}$ and let $A \in$ $L^{\infty}\left(\mathbb{R}^{n} ; \mathcal{L}\left(\mathbb{C}^{n}\right)\right)$. Consider the sesquilinear form $\mathfrak{l}^{A}: H^{1}\left(\mathbb{R}^{n}\right) \times H^{1}\left(\mathbb{R}^{n}\right) \rightarrow \mathbb{C}$ defined by

$$
\mathfrak{l}^{A}[u, v]:=\int_{\mathbb{R}^{n}}\langle A(x) \nabla u(x), \nabla v(x)\rangle_{\mathbb{C}^{n}} d x
$$

for $u, v \in H^{1}\left(\mathbb{R}^{n}\right)$. Suppose that $\mathfrak{l}^{A}$ satisfies the Gårding inequality

$$
\operatorname{Re}\left(\mathfrak{l}^{A}[u, u]\right) \geq \kappa^{A}\|\nabla u\|_{2}^{2}
$$

for all $u \in H^{1}\left(\mathbb{R}^{n}\right)$, for some $\kappa^{A}>0$. A well-known representation theorem from classical form theory (c.f. [17, Thm. VI.2.1]) asserts the existence of an associated operator $L: D(L) \subset L^{2}\left(\mathbb{R}^{n}\right) \rightarrow L^{2}\left(\mathbb{R}^{n}\right)$ for which

$$
\mathfrak{l}^{A}[u, v]=\langle L u, v\rangle_{2}
$$

for all $v \in H^{1}\left(\mathbb{R}^{n}\right)$ and $u$ in the domain of $L$,

$$
D(L)=\left\{u \in H^{1}\left(\mathbb{R}^{n}\right): \exists w \in L^{2}\left(\mathbb{R}^{n}\right) \text { s.t. } \mathfrak{l}^{A}[u, v]=\langle w, v\rangle_{2} \forall v \in H^{1}\left(\mathbb{R}^{n}\right)\right\}
$$

The operator $L$ is a densely defined maximal accretive operator that is denoted

$$
L=-\operatorname{div}(A \nabla)
$$

Due to the accretivity of the operator, it is possible to define a square root operator $\sqrt{L}$, with core $D(L)$, that satisfies $\sqrt{L} \cdot \sqrt{L}=L$. The Kato square root problem asks: what is the domain of definition of the square root operator? This problem, first posed by Tosio Kato over 50 years ago, was conjectured to have the following solution.

Theorem 1.1 (Kato Square Root) The domain of $\sqrt{L}$ is $D(\sqrt{L})=H^{1}\left(\mathbb{R}^{n}\right)$ and for any $u \in H^{1}\left(\mathbb{R}^{n}\right)$

$$
\|\sqrt{L} u\|_{2} \simeq\|\nabla u\|_{2}
$$

This long-standing problem withstood solution until 2002 where it was proved using local T(b) methods by Steve Hofmann, Michael Lacey and Alan McIntosh in [15] under the additional assumption of Gaussian heat kernel bounds and in full generality by Pascal Auscher, Steve Hofmann, Michael Lacey, Alan McIntosh and Phillipe Tchamitchian in [4]. We will be interested in an alternate method of proof that was built from similar principles and appeared a few years later. 
Let $\Pi:=\Gamma+\Gamma^{*}$ be a Dirac-type operator on a Hilbert space $\mathcal{H}$ and $\Pi_{B}:=$ $\Gamma+B_{1} \Gamma^{*} B_{2}$ be a perturbation of $\Pi$ by bounded operators $B_{1}$ and $B_{2}$. Typically, $\Pi$ is considered to be a first-order system acting on $\mathcal{H}:=L^{2}\left(\mathbb{R}^{n} ; \mathbb{C}^{N}\right)$ for some $n, N \in \mathbb{N}^{*}$ and the perturbations $B_{1}$ and $B_{2}$ are multiplication by matrix-valued functions $B_{1}, B_{2} \in L^{\infty}\left(\mathbb{R}^{n} ; \mathcal{L}\left(\mathbb{C}^{N}\right)\right)$. In their seminal paper [6], Axelsson, Keith and McIntosh developed a general framework for proving that the perturbed operator $\Pi_{B}$ possessed a bounded holomorphic functional calculus. This ultimately amounted to obtaining square function estimates of the form

$$
\int_{0}^{\infty}\left\|Q_{t}^{B} u\right\|^{2} \frac{d t}{t} \simeq\|u\|^{2}
$$

where $Q_{t}^{B}:=t \Pi_{B}\left(I+t^{2} \Pi_{B}^{2}\right)^{-1}$ and $u$ is contained in the range $\overline{R\left(\Pi_{B}\right)}$. They proved that this estimate would follow entirely from a set of simple conditions imposed upon the operators $\Gamma, B_{1}$ and $B_{2}$, labelled (H1)-(H8). Then, by checking this list of simple conditions, the Axelsson-Keith-McIntosh framework, or AKM framework by way of abbreviation, could be used to conclude that the particular selection of operators

$$
\Gamma:=\left(\begin{array}{cc}
0 & 0 \\
\nabla & 0
\end{array}\right), \quad B_{1}=I, \quad B_{2}=\left(\begin{array}{ll}
I & 0 \\
0 & A
\end{array}\right),
$$

defined on $L^{2}\left(\mathbb{R}^{n}\right) \oplus L^{2}\left(\mathbb{R}^{n} ; \mathbb{C}^{n}\right)$, would satisfy (3) and therefore $\Pi_{B}$ would possess a bounded holomorphic functional calculus. The Kato square root estimate then followed almost trivially from this.

Many classical problems from harmonic analysis will have a direct counterpart in the Schrödinger operator setting. Adhering with this theme, one can consider the Kato square root problem with potential. Let $V: \mathbb{R}^{n} \rightarrow \mathbb{C}$ be a measurable function that is contained in $L_{l o c}^{1}\left(\mathbb{R}^{n}\right) . V$ can be viewed as a densely defined closed multiplication operator on $L^{2}\left(\mathbb{R}^{n}\right)$ with domain

$$
D(V)=\left\{u \in L^{2}\left(\mathbb{R}^{n}\right): V \cdot u \in L^{2}\left(\mathbb{R}^{n}\right)\right\}
$$

The density of $D(V)$ follows from the condition $V \in L_{l o c}^{1}\left(\mathbb{R}^{n}\right)$. Define the subspace

$$
H^{1, V}\left(\mathbb{R}^{n}\right):=H^{1}\left(\mathbb{R}^{n}\right) \cap D\left(V^{\frac{1}{2}}\right):=\left\{u \in H^{1}\left(\mathbb{R}^{n}\right): V^{\frac{1}{2}} \cdot u \in L^{2}\left(\mathbb{R}^{n}\right)\right\} .
$$

Here the complex square root $V^{\frac{1}{2}}$ is defined via the principal branch cut along the negative real axis. It is easy to see that $H^{1, V}\left(\mathbb{R}^{n}\right)$ is dense in $L^{2}\left(\mathbb{R}^{n}\right)$ since $C_{0}^{\infty}\left(\mathbb{R}^{n}\right) \subset$ $H^{1, V}\left(\mathbb{R}^{n}\right)$.

Let $A \in L^{\infty}\left(\mathbb{R}^{n} ; \mathcal{L}\left(\mathbb{C}^{n}\right)\right)$ be as before with (1) satisfied for some $\kappa^{A}>0$. Consider the sesquilinear form $\mathfrak{l}_{V}^{A}: H^{1, V}\left(\mathbb{R}^{n}\right) \times H^{1, V}\left(\mathbb{R}^{n}\right) \rightarrow \mathbb{C}$ defined through

$$
\mathfrak{l}_{V}^{A}[u, v]:=\mathfrak{l}^{A}[u, v]+\int_{\mathbb{R}^{n}} V(x) u(x) \bar{v}(x) d x
$$


for $u, v \in H^{1, V}\left(\mathbb{R}^{n}\right)$. Suppose that there exists some $\kappa_{V}^{A}>0$ for which $\mathfrak{l}_{V}^{A}$ satisfies the associated Gårding inequality

$$
\operatorname{Re}\left(\mathfrak{l}_{V}^{A}[u, u]\right) \geq \kappa_{V}^{A}\left(\left\|V^{\frac{1}{2}} u\right\|_{2}^{2}+\|\nabla u\|_{2}^{2}\right)
$$

for all $u \in H^{1, V}\left(\mathbb{R}^{n}\right)$.

Remark 1.1 If the range of $V$ is contained in some sector

$$
S_{\mu^{+}}:=\{z \in \mathbb{C} \cup\{\infty\}:|\arg (z)| \leq \mu \text { or } z=0, \infty\}
$$

for some $\mu \in\left[0, \frac{\pi}{2}\right.$ ), then (6) will follow automatically from (1).

Once again, the accretivity of $\mathfrak{l}_{V}^{A}$ implies the existence of a maximal accretive operator associated with this form denoted by

$$
L+V=-\operatorname{div}(A \nabla)+V
$$

and defined on

$$
\begin{aligned}
& D(L+V) \\
& \quad=\left\{u \in H^{1, V}\left(\mathbb{R}^{n}\right): \exists w \in L^{2}\left(\mathbb{R}^{n}\right) \text { s.t. } \mathfrak{l}_{V}^{A}[u, v]=\langle w, v\rangle_{2} \forall v \in H^{1, V}\left(\mathbb{R}^{n}\right)\right\} .
\end{aligned}
$$

For $\alpha \in[1,2]$, define $\mathcal{W}_{\alpha}$ to be the class of all $V \in L_{\text {loc }}^{1}\left(\mathbb{R}^{n}\right)$ for which

$$
[V]_{\alpha}:=\sup _{u \in D(|V|-\Delta)} \frac{\left\||V|^{\frac{\alpha}{2}} u\right\|_{2}+\left\|(-\Delta)^{\frac{\alpha}{2}} u\right\|_{2}}{\left\|(|V|-\Delta)^{\frac{\alpha}{2}} u\right\|_{2}}<\infty .
$$

As will be proved in Sect. 5.2, the collection of potential classes $\left\{\mathcal{W}_{\alpha}\right\}_{\alpha \in[1,2]}$ is decreasing. The largest class $\mathcal{W}_{1}$ consists of all locally integrable potentials with no additional restrictions and the smallest class $\mathcal{W}_{2}$ contains $R H_{2}$ in any dimension and $L^{\frac{n}{2}}\left(\mathbb{R}^{n}\right)$ in dimension $n>4$. In this paper, our aim is to prove the potential dependent Kato estimate as presented in the following theorem.

Theorem 1.2 (Kato with Potential) Let $V \in \mathcal{W}_{\alpha}$ for some $\alpha \in(1,2]$ and $A \in$ $L^{\infty}\left(\mathbb{R}^{n} ; \mathcal{L}\left(\mathbb{C}^{n}\right)\right)$. Suppose that the Gårding inequalities (1) and (6) are both satisfied with constants $\kappa^{A}>0$ and $\kappa_{V}^{A}>0$ respectively. There exists a constant $C_{V}>0$ such that

$$
C_{V}^{-1}\left(\left\|V^{\frac{1}{2}} u\right\|_{2}+\|\nabla u\|_{2}\right) \leq\|\sqrt{L+V} u\|_{2} \leq C_{V}\left(\left\|V^{\frac{1}{2}} u\right\|_{2}+\|\nabla u\|_{2}\right)
$$

for all $u \in H^{1, V}\left(\mathbb{R}^{n}\right)$. Moreover, the constant $C_{V}$ depends on $V$ and $\alpha$ through

$$
C_{V}=\tilde{C}_{V}(\alpha-1)^{-1}\left(1+[V]_{\alpha}^{2}\right),
$$


where $\tilde{C}_{V}$ only depends on $V$ through $\kappa_{V}^{A}$ and is independent of $\alpha$.

This theorem is actually a statement concerning the domain of the square root operator $\sqrt{L+V}$. Indeed, (KP) implies the equality

$$
D(\sqrt{L+V})=H^{1, V}\left(\mathbb{R}^{n}\right) .
$$

Remark 1.2 The above theorem tells us, in particular, that the Kato estimate with potential, (KP), is valid for any potential $V$ with range contained in $S_{\mu+}$ for some $\mu \in\left[0, \frac{\pi}{2}\right)$ and such that $|V|$ is either contained in $\mathrm{RH}_{2}$ for any dimension or in $L^{\frac{n}{2}}\left(\mathbb{R}^{n}\right)$ in dimension $n>4$. In [2, p. 3], it is suggested but not proved that $[V]_{\alpha}<\infty$ will occur provided that $|V| \in R H_{q}$ with $q>\alpha$. If this is indeed the case then Theorem 1.2 will imply that the Kato estimate is true for any potential $V$ with range contained in $S_{\mu+}$ for some $\mu \in\left[0, \frac{\pi}{2}\right)$ and with $|V|$ contained in $R H_{1}=A_{\infty}$.

In direct analogy to the potential free case, the Kato problem with potential will be solved by constructing appropriate potential dependent Dirac-type operators and demonstrating that they retain a bounded holomorphic functional calculus under perturbation. In particular, this strategy will be applied to the Dirac-type operator

$$
\Pi_{|V|^{\frac{1}{2}}}:=\Gamma_{|V|^{\frac{1}{2}}}+\Gamma_{|V|^{\frac{1}{2}}}^{*}:=\left(\begin{array}{ccc}
0 & 0 & 0 \\
|V|^{\frac{1}{2}} & 0 & 0 \\
\nabla & 0 & 0
\end{array}\right)+\left(\begin{array}{ccc}
0|V|^{\frac{1}{2}} & -\operatorname{div} \\
0 & 0 & 0 \\
0 & 0 & 0
\end{array}\right)
$$

defined on $L^{2}\left(\mathbb{R}^{n}\right) \oplus L^{2}\left(\mathbb{R}^{n}\right) \oplus L^{2}\left(\mathbb{R}^{n} ; \mathbb{C}^{n}\right)$, under the perturbation

$$
B_{1}=I, \quad B_{2}:=\left(\begin{array}{ccc}
I & 0 & 0 \\
0 & e^{i \cdot \arg V} & 0 \\
0 & 0 & A
\end{array}\right) .
$$

It should be observed that the operator $\Gamma_{|V|^{\frac{1}{2}}}$ is not first-order homogeneous due to the presence of the zero-order potential term. It will therefore not necessarily satisfy the two conditions of the original AKM framework that are intended to capture the firstorder homogeneity property, the cancellation and coercivity conditions, (H7) and (H8). As such, the original AKM framework cannot be directly applied. The key difficulty in proving our result is then to alter the original framework in order to allow for such operators. In particular, a non-homogeneous version of the Axelsson-Keith-McIntosh framework will be developed to handle operators of the form

$$
\Gamma_{J}:=\left(\begin{array}{ccc}
0 & 0 & 0 \\
J & 0 & 0 \\
D & 0 & 0
\end{array}\right),
$$

where $D$ is a homogeneous first-order differential operator and $J$ is a possibly nonhomogeneous differential operator of order less than or equal to one. The technical 
challenge presented by the inclusion of the non-homogeneous part $J$ will be overcome by separating our square function norm into components and demonstrating that the non-homogeneous term will allow for the first two components to be bounded while the third component can be bound using an argument similar to the classical argument of [6].

Since the operator $\Gamma_{J}$ is of a more general form than $\Gamma_{|V|^{\frac{1}{2}}}$, the non-homogeneous AKM framework that we develop will have applications not confined to zero-order scalar potentials. Indeed, the non-homogeneous framework will also be used to prove Kato estimates for systems of equations with zero-order potential and for scalar equations with first-order potentials.

The structure of this paper is as follows. Section 2 is quite classical in nature. It provides a brief survey of the natural functional calculus for bisectorial operators. It also describes how to prove the boundedness of holomorphic functional calculus from square function estimates for a bisectorial operator. These classical proofs are repeated here to track the dependence of the estimates on relevant constants. This will be required to determine the potential dependence of the constants in Theorem 1.2. Section 3 describes the non-homogeneous AKM framework and states the main results associated with it. Section 4 contains most of the technical machinery and is dedicated to a proof of our main result. Section 5 will apply the non-homogeneous AKM framework to the scalar Kato problem with potential, the Kato problem for systems with zero-order potential and the scalar Kato problem with first-order potential. It is here that a proof of Theorem 1.2 will be completed. Finally, in Sect. 6, we will provide a meta-discussion on the proof techniques used and compare our work with what has been previously accomplished on non-homogeneous Kato-type estimates.

\section{Preliminaries}

Let's outline the construction of the natural functional calculus associated with a bisectorial operator. The treatment of functional calculi found here follows closely to [14] with significant detail omitted. Appropriate changes are made to account for the fact that we consider bisectorial operators instead of sectorial operators. Other thorough treatments of functional calculus for sectorial operators can be found in [1] or [16].

For $\mu \in[0, \pi)$ define the open and closed sectors

$$
S_{\mu+}^{o}:=\left\{\begin{array}{cc}
\{z \in \mathbb{C} \backslash\{0\}:|\arg (z)|<\mu\} \mu \in(0, \pi) \\
(0, \infty) & \mu=0
\end{array}\right.
$$

and

$$
S_{\mu+}:=\left\{\begin{array}{cc}
\{z \in \mathbb{C} \cup\{\infty\}:|\arg (z)| \leq \mu \text { or } z=0, \infty\} & \mu \in(0, \pi) \\
{[0, \infty]} & \mu=0 .
\end{array}\right.
$$


Then, for $\mu \in\left[0, \frac{\pi}{2}\right)$, define the open and closed bisectors

$$
S_{\mu}^{o}:=\left(S_{\mu+}^{o}\right) \cup\left(-S_{\mu+}^{o}\right)
$$

and

$$
S_{\mu}:=\left(S_{\mu+}\right) \cup\left(-S_{\mu+}\right)
$$

respectively. Throughout this section we consider bisectorial operators defined on a Hilbert space $\mathcal{H}$ with norm and inner product denoted by $\|\cdot\|$ and $\langle\cdot, \cdot\rangle$ respectively.

Definition 2.1 (Bisectorial Operator) A linear operator $T: D(T) \subseteq \mathcal{H} \rightarrow \mathcal{H}$ is said to be $\omega$-bisectorial for $\omega \in\left[0, \frac{\pi}{2}\right)$ if the spectrum $\sigma(T)$ is contained in the bisector $S_{\omega}$ and if for any $\mu \in\left(\omega, \frac{\pi}{2}\right)$, there exists $C_{\mu}>0$ such that the resolvent bound

$$
|\zeta|\left\|(\zeta I-T)^{-1}\right\| \leq C_{\mu}
$$

holds for all $\zeta \in \mathbb{C} \backslash S_{\mu}$. T is said to be bisectorial if it is $\omega$-bisectorial for some $\omega \in\left[0, \frac{\pi}{2}\right)$.

Sectorial operators are defined identically except with the sector $S_{\mu+}$ performing the role of the bisector $S_{\mu}$. An important fact concerning bisectorial operators is the following decomposition result.

Proposition 2.1 ([7, Thm. 3.8]) Let $T: D(T) \subset \mathcal{H} \rightarrow \mathcal{H}$ be a bisectorial operator Then $T$ is necessarily densely defined and the Hilbert space $\mathcal{H}$ admits the following decomposition

$$
\mathcal{H}=N(T) \oplus \overline{R(T)}
$$

Let $T$ be an $\omega$-bisectorial operator for $\omega \in\left[0, \frac{\pi}{2}\right)$ and $\mu \in\left(\omega, \frac{\pi}{2}\right)$. Let $\mathcal{M}\left(S_{\mu}^{o}\right)$ denote the algebra of all meromorphic functions on the open bisector $S_{\mu}^{o}$ and define the following subalgebras,

$$
\begin{aligned}
& H\left(S_{\mu}^{o}\right):=\left\{f \in \mathcal{M}\left(S_{\mu}^{o}\right): f \text { holomorphic on } S_{\mu}^{o}\right\} \\
& H^{\infty}\left(S_{\mu}^{o}\right):=\left\{f \in H\left(S_{\mu}^{o}\right):\|f\|_{\infty}:=\sup _{z \in S_{\mu}^{o}}|f(z)|<\infty\right\}
\end{aligned}
$$

and

$$
H_{0}^{\infty}\left(S_{\mu}^{o}\right):=\left\{f \in H^{\infty}\left(S_{\mu}^{o}\right): \exists C, \alpha>0 \text { s.t. }|f(z)| \leq C \cdot \frac{|z|^{\alpha}}{1+|z|^{2 \alpha}} \forall z \in S_{\mu}^{o}\right\}
$$


For any $f \in H_{0}^{\infty}\left(S_{\mu}^{o}\right)$, one can associate an operator $f(T)$ as follows. For $u \in \mathcal{H}$, define

$$
f(T) u:=\frac{1}{2 \pi i} \oint_{\gamma} f(z)(z I-T)^{-1} u d z,
$$

where the curve

$$
\gamma:=\left\{ \pm r e^{ \pm i v}: 0 \leq r<\infty\right\}
$$

for some $v \in(\omega, \mu)$ is traversed anticlockwise. Using the resolvent bounds of our operator in combination with the size estimates for functions in $H_{0}^{\infty}\left(S_{\mu}^{o}\right)$, it can easily be shown that the operator $f(T)$ is well-defined. Moreover, it can also be proved that this association constitutes an algebra homomorphism.

Theorem 2.1 ([14, Lem. 2.3.1]) The map $\Phi_{0}^{T}: H_{0}^{\infty}\left(S_{\mu}^{o}\right) \rightarrow \mathcal{L}(\mathcal{H})$ defined through

$$
\Phi_{0}^{T}(f):=f(T)
$$

is a well-defined algebra homomorphism. Moreover, it is independent of the value of $v$.

Since the functions in $H_{0}^{\infty}\left(S_{\mu}^{o}\right)$ approach zero at the origin we should expect that the null space of the newly formed operator will be larger than the null space of the original operator. This is indeed the case as stated in the below proposition.

Proposition 2.2 ([14, Thm. 2.3.3]) For a bisectorial operator $T: D(T) \subseteq \mathcal{H} \rightarrow \mathcal{H}$, the null-space inclusion

$$
N(T) \subseteq N(f(T))
$$

holds for all $f \in H_{0}^{\infty}\left(S_{\mu}^{o}\right)$.

Define the subalgebra of functions

$$
\mathcal{E}\left(S_{\mu}^{o}\right):=H_{0}^{\infty}\left(S_{\mu}^{o}\right) \oplus\left\langle(z+i)^{-1}\right\rangle \oplus\langle 1\rangle .
$$

$\Phi_{0}^{T}$ has an extension

$$
\Phi_{p}^{T}: \mathcal{E}\left(S_{\mu}^{o}\right) \rightarrow \mathcal{L}(H)
$$

defined through

$$
\Phi_{p}^{T}(g):=g(T):=f(T)+c \cdot(T+i)^{-1}+d \cdot I
$$

for $g=f+c \cdot(z+i)^{-1}+d \in \mathcal{E}\left(S_{\mu}^{o}\right)$, where $f \in H_{0}^{\infty}\left(S_{\mu}^{o}\right)$ and $c, d \in \mathbb{C}$. 
Theorem 2.2 ([14, Thm. 2.3.3]) The map $\Phi_{p}^{T}$ is an algebra homomorphism called the primary functional calculus associated with $T$.

This map can be extended once more through the process of regularization. A function $f \in \mathcal{M}\left(S_{\mu}^{o}\right)$ is said to be regularizable with respect to the primary functional calculus $\Phi_{p}^{T}: \mathcal{E}\left(S_{\mu}^{o}\right) \rightarrow \mathcal{L}(\mathcal{H})$ if there exists $e \in \mathcal{E}\left(S_{\mu}^{o}\right)$ such that $e(T)$ is injective and $e \cdot f \in \mathcal{E}\left(S_{\mu}^{o}\right)$. The notation $\mathcal{E}\left(S_{\mu}^{o}\right)_{r}$ will be used to denote the algebra of regularizable functions. Let $\mathcal{C}(\mathcal{H})$ denote the set of closed operators from $\mathcal{H}$ to itself. Then define the extension

$$
\Phi^{T}: \mathcal{E}\left(S_{\mu}^{o}\right)_{r} \rightarrow \mathcal{C}(H)
$$

through

$$
\Phi^{T}(f):=f(T):=\Phi_{p}^{T}(e)^{-1} \cdot \Phi_{p}^{T}(e \cdot f)
$$

for $f \in \mathcal{E}\left(S_{\mu}^{o}\right)_{r}$ and $e \in \mathcal{E}\left(S_{\mu}^{o}\right)$ a regularizing function for $f$. This definition is independent of the chosen regularizer $e$ for $f$ and therefore $\Phi^{T}$ is well-defined. We have the following important theorem that establishes the desired properties of a functional calculus for this extension. Thus the map $\Phi^{T}$ is known as the natural functional calculus for the operator $T$.

Theorem 2.3 ([14, Thm. 1.3.2]) Let T be an $\omega$-bisectorial operator on a Hilbert space $\mathcal{H}$ for some $\omega \in\left[0, \frac{\pi}{2}\right)$. Let $\mu \in\left(\omega, \frac{\pi}{2}\right)$. The following assertions hold.

(1) $\mathbf{1}(T)=I$ and $(z)(T)=T$, where $1: S_{\mu}^{o} \rightarrow \mathbb{C}$ is the constant function defined by $\boldsymbol{I}(z):=1$ for $z \in S_{\mu}^{o}$.

(2) Let $f, g \in \mathcal{E}\left(S_{\mu}^{o}\right)_{r}$. Then

$$
f(T)+g(T) \subset(f+g)(T), \quad f(T) g(T) \subset(f \cdot g)(T)
$$

and $D(f(T) g(T))=D((f \cdot g)(T)) \cap D(g(T))$. One will have equality in these relations if $g(T) \in \mathcal{L}(H)$.

The ensuing definition plays a vital role in the solution method to the Kato square root problem using the AKM framework.

Definition 2.2 Let $0 \leq \omega<\mu<\frac{\pi}{2}$. An $\omega$-bisectorial operator $T: D(T) \subset \mathcal{H} \rightarrow \mathcal{H}$ is said to have a bounded $H^{\infty}\left(S_{\mu}^{o}\right)$-functional calculus if there exists $c>0$ such that

$$
\|f(T)\| \leq c \cdot\|f\|_{\infty}
$$

for all $f \in H_{0}^{\infty}\left(S_{\mu}^{o}\right) . T$ is said to have a bounded holomorphic functional calculus if it has a bounded $H^{\infty}\left(S_{\mu}^{o}\right)$-functional calculus for some $\mu$. 
Remark 2.1 Note that a more intuitive definition for a bounded $H^{\infty}\left(S_{\mu}^{o}\right)$-functional calculus would be to require that (11) hold for all $f \in H^{\infty}\left(S_{\mu}^{o}\right)$. Unfortunately at this stage it is impossible to ascertain whether $H^{\infty}\left(S_{\mu}^{o}\right) \subset \mathcal{E}\left(S_{\mu}^{o}\right)_{r}$. When this inclusion does not hold, the operator $f(T)$ will not be well-defined for all $f \in H^{\infty}\left(S_{\mu}^{o}\right)$. If $T$ so happens to be injective, then each $f \in H^{\infty}\left(S_{\mu}^{o}\right)$ is in fact regularizable by $z\left(1+z^{2}\right)^{-1}$ and the estimate (11) makes sense for all $f \in H^{\infty}\left(S_{\mu}^{o}\right)$. Fortunately, in this situation the two definitions coincide. That is, (11) will be true for all $f \in H_{0}^{\infty}\left(S_{\mu}^{o}\right)$ if and only if it is true for all $f \in H^{\infty}\left(S_{\mu}^{o}\right)$ when $T$ is injective.

Definition 2.3 (Square Function Norms) Let $\psi \in H_{0}^{\infty}\left(S_{\mu}^{o}\right)$. For $t>0$, define $\psi_{t}$ : $S_{\mu}^{o} \rightarrow \mathbb{C}$ to be the function $\psi_{t}(z):=\psi(t z)$ for $z \in S_{\mu}^{o}$. The square function norm associated with $\psi$ and $T$ is defined through

$$
\|u\|_{\psi, T}:=\left(\int_{0}^{\infty}\left\|\psi_{t}(T) u\right\|^{2} \frac{d t}{t}\right)^{\frac{1}{2}}
$$

for $u \in \mathcal{H}$. Let $q: S_{\mu}^{o} \rightarrow \mathbb{C}$ be the function given by

$$
q(z):=\frac{z}{1+z^{2}}, \quad z \in S_{\mu}^{o}
$$

$\|\cdot\|_{q, T}$ is called the canonical square function norm for the operator $T$.

For injective $T$, true to their name, the square function norms $\|\cdot\|_{\psi, T}$, for $\psi \in$ $H_{0}^{\infty}\left(S_{\mu}^{o}\right)$ not identically equal to zero on either $S_{\mu+}^{o}$ or $\left(-S_{\mu+}^{o}\right)$, are indeed norms on $\mathcal{H}$. For non-injective $T$, however, they are at most seminorms on $\mathcal{H}$ and will only be norms following a restriction to the subspace $\overline{R(T)}$.

Definition 2.4 (Square Function Estimates) A bisectorial operator $T$ on a Hilbert space $\mathcal{H}$ is said to satisfy square function estimates if there exists a constant $C_{S F}>0$ such that

$$
C_{S F}^{-\frac{1}{2}} \cdot\|u\| \leq\|u\|_{q, T} \leq C_{S F}^{\frac{1}{2}} \cdot\|u\|
$$

for all $u \in \overline{R(T)}$.

The above definition is the same as saying that the canonical square function norm $\|\cdot\|_{q, T}$ is norm equivalent to $\|\cdot\|_{\mathcal{H}}$ when restricted to the Hilbert subspace $\overline{R(T)}$.

Remark 2.2 The use of the canonical norm $\|\cdot\|_{q, T}$ in the above definition of square function estimates is somewhat arbitrary. Indeed, it can be swapped with $\|\cdot\|_{\psi, T}$ for any $\psi \in H_{0}^{\infty}\left(S_{\mu}^{o}\right)$ not identically equal to zero on either $S_{\mu+}^{o}$ or $\left(-S_{\mu+}^{o}\right)$. This follows from the equivalence of these two norms as stated in [14, Thm. 7.3.1]. The implicit constant in the norm equivalence will depend on the function $\psi$ under consideration. 
Proposition 2.3 (Resolution of the Identity) For $\psi \in H_{0}^{\infty}\left(S_{\mu}^{o}\right)$ and any $u \in \mathcal{H}$,

$$
c_{\psi}\left(I-\mathbb{P}_{N(T)}\right) u=\int_{0}^{\infty} \psi_{t}(T)^{2} u \frac{d t}{t}
$$

where $\mathbb{P}_{N(T)}$ denotes the projection operator onto the subspace $N(T)$ and

$$
c_{\psi}:=\int_{0}^{\infty} \psi(t)^{2} \frac{d t}{t} .
$$

Proof Equality follows from Proposition 2.2 for $u \in N(T)$. For $u \in \overline{R(T)}$ this is given by Theorem 5.2.6 of [14] in the sectorial case. The bisectorial case can be proved similarly.

Corollary 2.1 Suppose that $T$ is self-adjoint and $\psi \in H_{0}^{\infty}\left(S_{\mu}^{o}\right)$. Then for any $u \in \mathcal{H}$,

$$
\int_{0}^{\infty}\left\|\psi_{t}(T) u\right\|^{2} \frac{d t}{t} \leq c_{\psi}\|u\|^{2}
$$

where $c_{\psi}$ is as defined in the previous proposition. Equality will hold if $u \in \overline{R(T)}$.

Proof As $T$ is self-adjoint, if follows from the definition of $\psi_{t}(T)$ that it must also be self-adjoint. On expanding the square function norm,

$$
\begin{aligned}
\int_{0}^{\infty}\left\|\psi_{t}(T) u\right\|^{2} \frac{d t}{t} & =\int_{0}^{\infty}\left\langle\psi_{t}(T) u, \psi_{t}(T) u\right\rangle \frac{d t}{t} \\
& =\left\langle u, \int_{0}^{\infty} \psi_{t}(T)^{2} u \frac{d t}{t}\right\rangle .
\end{aligned}
$$

The previous proposition then gives

$$
\begin{aligned}
\int_{0}^{\infty}\left\|\psi_{t}(T) u\right\|^{2} \frac{d t}{t} & =\left\langle u, c_{\psi}\left(I-\mathbb{P}_{N(T)}\right) u\right\rangle \\
& \leq c_{\psi}\|u\|^{2}
\end{aligned}
$$

Equality will clearly hold in the above if $u \in \overline{R(T)}$.

Theorem 2.4 Let $T$ be an $\omega$-bisectorial operator on $\mathcal{H}$ for $\omega \in\left[0, \frac{\pi}{2}\right)$. Suppose that $T$ satisfies square function estimates with constant $C_{S F}>0$. Then $T$ must have a bounded $H^{\infty}\left(S_{\mu}^{o}\right)$-holomorphic functional calculus for any $\mu \in\left(\omega, \frac{\pi}{2}\right)$. In particular, there exists a constant $c>0$, independent of $T$, such that

$$
\|f(T)\| \leq\left(c \cdot C_{S F} \cdot C_{\mu}\right) \cdot\|f\|_{\infty}
$$

for all $f \in H_{0}^{\infty}\left(S_{\mu}^{o}\right)$, where $C_{\mu}>0$ is the constant from the resolvent estimate (10). 
Proof Let $f \in H_{0}^{\infty}\left(S_{\mu}^{o}\right)$. For $u \in N(T)$, the bound

$$
\|f(T) u\| \leq\left(c \cdot C_{S F} \cdot C_{\mu} \cdot\|f\|_{\infty}\right) \cdot\|u\|
$$

follows trivially from Proposition 2.2 for any $c>0$. Fix $u \in \overline{R(T)}$. On applying the lower square function estimate to $f(T) u \in \overline{R(T)}$,

$$
\begin{aligned}
\|f(T) u\|^{2} & \leq C_{S F} \int_{0}^{\infty}\left\|q_{s}(T) f(T) u\right\|^{2} \frac{d s}{s} \\
& =2 C_{S F} \int_{0}^{\infty}\left\|q_{s}(T) f(T) \int_{0}^{\infty}\left(q_{t}(T)\right)^{2} u \frac{d t}{t}\right\|^{2} \frac{d s}{s} \\
& \leq 2 C_{S F} \int_{0}^{\infty}\left(\int_{0}^{\infty}\left\|q_{s}(T) f(T) q_{t}(T)\right\|\left\|q_{t}(T) u\right\| \frac{d t}{t}\right)^{2} \frac{d s}{s},
\end{aligned}
$$

where in the second line we used the resolution of the identity Proposition 2.3. The Cauchy-Schwarz inequality then gives

$\|f(T) u\|^{2} \leq 2 C_{S F} \int_{0}^{\infty}\left(\int_{0}^{\infty}\left\|q_{s}(T) f(T) q_{t}(T)\right\| \frac{d t}{t}\right)\left(\int_{0}^{\infty}\left\|q_{s}(T) f(T) q_{t}(T)\right\|\left\|q_{t}(T) u\right\|^{2} \frac{d t}{t}\right) \frac{d s}{s}$.

From the homomorphism property for the $H_{0}^{\infty}\left(S_{\mu}^{o}\right)$-functional calculus,

$$
q_{s}(T) f(T) q_{t}(T)=\left(q_{s} \cdot f \cdot q_{t}\right)(T) .
$$

Since our operator $T$ satisfies resolvent bounds with constant $C_{\mu}>0$,

$$
\begin{aligned}
\left\|q_{s}(T) f(T) q_{t}(T)\right\| & =\left\|\left(q_{s} \cdot f \cdot q_{t}\right)(T)\right\| \\
& =\frac{1}{2 \pi}\left\|\int_{\gamma}\left(q_{s} \cdot f \cdot q_{t}\right)(z)(T-z I)^{-1} d z\right\| \\
& \leq \frac{C_{\mu}}{2 \pi} \cdot\|f\|_{\infty} \cdot \int_{\gamma}\left|q_{s}(z)\right|\left|q_{t}(z)\right| \frac{|d z|}{|z|} .
\end{aligned}
$$

On noting that $q \in H_{0}^{\infty}\left(S_{\mu}^{o}\right)$,

$$
\left\|q_{s}(T) f(T) q_{t}(T)\right\| \leq c \cdot C_{\mu} \cdot\|f\|_{\infty} \cdot \int_{\gamma} \frac{|s z|^{\alpha}}{1+|s z|^{2 \alpha}} \frac{|t z|^{\alpha}}{1+|t z|^{2 \alpha}} \frac{|d z|}{|z|}
$$

for some $\alpha>0$ and constant $c>0$ independent of $T$. Thus we obtain the estimate $\left\|q_{s}(T) f(T) q_{t}(T)\right\| \leq c \cdot C_{\mu} \cdot\|f\|_{\infty} \cdot\left\{\begin{array}{l}\left(\frac{t}{s}\right)^{\alpha}\left(1+\log \left(\frac{s}{t}\right)\right) \text { for } 0<t \leq s<\infty \\ \left(\frac{s}{t}\right)^{\alpha}\left(1+\log \left(\frac{t}{s}\right)\right) \text { for } 0<s<t<\infty\end{array}\right.$ 
where the value of the $T$ independent constant $c$ is allowed to change. This then implies that

$\sup _{s>0} \int_{0}^{\infty}\left\|q_{s}(T) f(T) q_{t}(T)\right\| \frac{d t}{t}, \quad \sup _{t>0} \int_{0}^{\infty}\left\|q_{s}(T) f(T) q_{t}(T)\right\| \frac{d s}{s} \leq c \cdot C_{\mu} \cdot\|f\|_{\infty}$.

On applying this estimate to (15),

$$
\begin{aligned}
\|f(T) u\|^{2} & \leq c \cdot C_{\mu} \cdot C_{S F} \cdot\|f\|_{\infty} \int_{0}^{\infty} \int_{0}^{\infty}\left\|q_{s}(T) f(T) q_{t}(T)\right\|\left\|q_{t}(T) u\right\|^{2} \frac{d t}{t} \frac{d s}{s} \\
& =c \cdot C_{\mu} \cdot C_{S F} \cdot\|f\|_{\infty} \int_{0}^{\infty}\left\|q_{t}(T) u\right\|^{2} \int_{0}^{\infty}\left\|q_{s}(T) f(T) q_{t}(T)\right\| \frac{d s}{s} \frac{d t}{t} \\
& \leq c^{2} \cdot C_{\mu}^{2} \cdot C_{S F} \cdot\|f\|_{\infty}^{2} \int_{0}^{\infty}\left\|q_{t}(T) u\right\|^{2} \frac{d t}{t} \\
& \lesssim c^{2} \cdot C_{\mu}^{2} \cdot C_{S F}^{2} \cdot\|f\|_{\infty}^{2}\|u\|^{2} .
\end{aligned}
$$

Finally, the following Kato-type estimate follows from a well-known classical argument.

Corollary 2.2 Suppose that the bisectorial operator $T$ satisfies square function estimates with constant $C_{S F}>0$ and the constant in the resolvent estimate (10) is $C_{\mu}>0$. Then there exists a constant $c>0$, independent of $T$, such that

$$
\left(c \cdot C_{S F} \cdot C_{\mu}\right)^{-1} \cdot\|T u\| \leq\left\|\sqrt{T^{2}} u\right\| \leq\left(c \cdot C_{S F} \cdot C_{\mu}\right) \cdot\|T u\|
$$

for any $u \in D(T)$.

Proof Consider the restriction $S:=\left.T\right|_{R(T)}$. $S$ is an injective bisectorial operator that satisfies square function estimates with constant $C_{S F}>0$. Since $S$ is injective it follows that $f(S)$ is well-defined for any $f \in H^{\infty}\left(S_{\mu}^{o}\right)$ by Remark 2.1. This allows us to define the operators $f_{1}(S)$ and $f_{2}(S)$, where the functions $f_{1}$ and $f_{2}$ are defined by

$$
f_{1}(z):=\frac{\sqrt{z^{2}}}{z} \text { and } f_{2}(z):=\frac{z}{\sqrt{z^{2}}} \text { for } z \in S_{\mu}^{o}
$$

The previous theorem allows us to deduce that both of these operators are norm bounded by $c \cdot C_{S F} \cdot C_{\mu}$ for some $T$ independent constant $c>0$. Applying the multiplicative part of Theorem 2.3 to the functions $f=f_{1}$ and $g(z)=z$ implies that

$$
\frac{\sqrt{S^{2}}}{S} \cdot S=\sqrt{S^{2}}
$$


on

$$
D(S)=D\left(\frac{\sqrt{S^{2}}}{S} \cdot S\right)=D\left(\sqrt{S^{2}}\right) \cap D(S)
$$

Similarly, applying the multiplicative part of Theorem 2.3 to $f=f_{2}$ and $g(z)=\sqrt{z^{2}}$ gives

$$
\frac{S}{\sqrt{S^{2}}} \cdot \sqrt{S^{2}}=S
$$

on

$$
D\left(\sqrt{S^{2}}\right)=D\left(\frac{S}{\sqrt{S^{2}}} \cdot \sqrt{S^{2}}\right)=D(S) \cap D\left(\sqrt{S^{2}}\right)
$$

Equations (18) and (20) together imply that the domains $D\left(\sqrt{S^{2}}\right)$ and $D(S)$ coincide and therefore both (17) and (19) will remain valid on all of $D(S)$.

Let $u \in D(T)$. Proposition 2.1 states that $u$ has the decomposition $u=u_{1} \oplus u_{2} \in$ $N(T) \oplus \overline{R(T)}$. Then

$$
\begin{aligned}
\|T u\| & =\left\|S u_{2}\right\| \\
& =\left\|\frac{S}{\sqrt{S^{2}}} \cdot \sqrt{S^{2}} u_{2}\right\| \\
& \leq c \cdot C_{S F} \cdot C_{\mu} \cdot\left\|\sqrt{S^{2}} u_{2}\right\| \\
& =c \cdot C_{S F} \cdot C_{\mu} \cdot\left\|\sqrt{T^{2}} u\right\|,
\end{aligned}
$$

where in the last line we used the fact that the functional calculus commutes with the restriction map as given in Proposition 2.6.5 of [14]. Also,

$$
\begin{aligned}
\left\|\sqrt{T^{2}} u\right\| & =\left\|\sqrt{S^{2}} u_{2}\right\| \\
& =\left\|\frac{\sqrt{S^{2}}}{S} \cdot S u_{2}\right\| \\
& \leq c \cdot C_{S F} \cdot C_{\mu} \cdot\left\|S u_{2}\right\| \\
& =c \cdot C_{S F} \cdot C_{\mu} \cdot\|T u\| .
\end{aligned}
$$




\section{Non-homogeneous Axelsson-Keith-Mclntosh}

In this section we describe how the Axelsson-Keith-McIntosh framework can be altered to account for non-homogeneous operators of the form (9). Our main results for this framework will also be stated.

\subsection{AKM Without Cancellation and Coercivity}

The operators that we wish to consider, $\Gamma_{J}$, will satisfy the first six conditions of [6]. However, they will not necessarily satisfy the cancellation condition (H7) and the coercivity condition (H8). It will therefore be fruitful to see what happens to the original AKM framework when the cancellation and coercivity conditions are removed.

Similar to the original result, we begin by assuming that we have operators that satisfy the hypotheses (H1)-(H3) from [6]. Recall these conditions for operators $\Gamma$, $B_{1}$ and $B_{2}$ on a Hilbert space $\mathcal{H}$ with norm $\|\cdot\|$ and inner product $\langle\cdot, \cdot\rangle$.

(H1) $\Gamma: D(\Gamma) \rightarrow \mathcal{H}$ is a closed, densely defined, nilpotent operator.

(H2) $B_{1}$ and $B_{2}$ satisfy the accretivity conditions

$$
\operatorname{Re}\left\langle B_{1} u, u\right\rangle \geq \kappa_{1}\|u\|^{2} \quad \text { and } \quad \operatorname{Re}\left\langle B_{2} v, v\right\rangle \geq \kappa_{2}\|v\|^{2}
$$

for all $u \in R\left(\Gamma^{*}\right)$ and $v \in R(\Gamma)$ for some $\kappa_{1}, \kappa_{2}>0$.

(H3) The operators $\Gamma$ and $\Gamma^{*}$ satisfy

$$
\Gamma^{*} B_{2} B_{1} \Gamma^{*}=0 \quad \text { and } \quad \Gamma B_{1} B_{2} \Gamma=0 .
$$

In $[6$, Sect. 4], the authors assume that they have operators that satisfy the hypotheses (H1)-(H3) and they derive several important operator theoretic consequences from only these hypotheses. As our operators $\Gamma, B_{1}$ and $B_{2}$ also satisfy (H1)-(H3), it follows that any result proved in $[6$, Sect. 4$]$ must also be true for our operators and can be used with impunity. In the interest of making this article as self-contained as possible, we will now restate any such result that is to be used in this paper.

Proposition 3.1 (The Hodge Decomposition, [6, Prop. 2.2]) Suppose that the operators $\left\{\Gamma, B_{1}, B_{2}\right\}$ satisfy $(H 1)-(H 3)$. Define the perturbation dependent operators

$$
\Gamma_{B}^{*}:=B_{1} \Gamma^{*} B_{2}, \quad \Gamma_{B}:=B_{2}^{*} \Gamma B_{1}^{*} \text { and } \Pi_{B}:=\Gamma+\Gamma_{B}^{*} .
$$

The Hilbert space $\mathcal{H}$ has the following Hodge decomposition into closed subspaces:

$$
\mathcal{H}=N\left(\Pi_{B}\right) \oplus \overline{R\left(\Gamma_{B}^{*}\right)} \oplus \overline{R(\Gamma)}
$$

Moreover, we have $N\left(\Pi_{B}\right)=N\left(\Gamma_{B}^{*}\right) \cap N(\Gamma)$ and $\overline{R\left(\Pi_{B}\right)}=\overline{R\left(\Gamma_{B}^{*}\right)} \oplus \overline{R(\Gamma)}$. When $B_{1}=B_{2}=I$ these decompositions are orthogonal, and in general the decompositions are topological. Similarly, there is also a decomposition

$$
\mathcal{H}=N\left(\Pi_{B}^{*}\right) \oplus \overline{R\left(\Gamma_{B}\right)} \oplus \overline{R\left(\Gamma^{*}\right)} .
$$


Proposition 3.2 ([6, Prop. 2.5]) Suppose that the operators $\left\{\Gamma, B_{1}, B_{2}\right\}$ satisfy $(H 1)$ (H3). The perturbed Dirac-type operator $\Pi_{B}$ is an $\omega$-bisectorial operator with $\omega:=$ $\frac{1}{2}\left(\omega_{1}+\omega_{2}\right)$ where

$$
\omega_{1}:=\sup _{u \in R\left(\Gamma^{*}\right) \backslash\{0\}}\left|\arg \left\langle B_{1} u, u\right\rangle\right|<\frac{\pi}{2}
$$

and

$$
\omega_{2}:=\sup _{u \in R(\Gamma) \backslash\{0\}}\left|\arg \left\langle B_{2} u, u\right\rangle\right|<\frac{\pi}{2} .
$$

The bisectoriality of $\Pi_{B}$ ensures that the following operators will be well-defined.

Definition 3.1 Suppose that the operators $\left\{\Gamma, B_{1}, B_{2}\right\}$ satisfy (H1)-(H3). For $t \in \mathbb{R} \backslash$ $\{0\}$, define the perturbation dependent operators

$$
\begin{aligned}
R_{t}^{B} & :=\left(I+i t \Pi_{B}\right)^{-1}, \quad P_{t}^{B}:=\left(I+t^{2}\left(\Pi_{B}\right)^{2}\right)^{-1} \\
Q_{t}^{B} & :=t \Pi_{B} P_{t}^{B} \quad \text { and } \quad \Theta_{t}^{B}:=t \Gamma_{B}^{*} P_{t}^{B} .
\end{aligned}
$$

When there is no perturbation, i.e. when $B_{1}=B_{2}=I$, the $B$ will dropped from the superscript or subscript. For example, instead of $\Theta_{t}^{I}$ or $\Pi_{I}$ the notation $\Theta_{t}$ and $\Pi$ will be employed.

Remark 3.1 An easy consequence of Proposition 3.2 is that the operators $R_{t}^{B}, P_{t}^{B}$ and $Q_{t}^{B}$ are all uniformly $\mathcal{H}$-bounded in $t$. Furthermore, on taking the Hodge decomposition Proposition 3.1 into account, it is clear that the operators $\Theta_{t}^{B}$ will also be uniformly $\mathcal{H}$-bounded in $t$.

The next result tells us how the operators $\Pi_{B}$ and $P_{t}^{B}$ interact with $\Gamma$ and $\Gamma_{B}^{*}$.

Lemma 3.1 ([6, Rmk. 4.5]) Suppose that the operators $\left\{\Gamma, B_{1}, B_{2}\right\}$ satisfy $(H 1)-(H 3)$. The following relations are true.

$$
\begin{aligned}
& \Pi_{B} \Gamma u=\Gamma_{B}^{*} \Pi_{B} u \quad \text { for all } u \in D\left(\Gamma_{B}^{*} \Pi_{B}\right), \\
& \Pi_{B} \Gamma_{B}^{*} u=\Gamma \Pi_{B} u \quad \text { for all } u \in D\left(\Gamma \Pi_{B}\right), \\
& \Gamma P_{t}^{B} u=P_{t}^{B} \Gamma u \quad \text { for all } u \in D(\Gamma), \quad \text { and } \\
& \Gamma_{B}^{*} P_{t}^{B} u=P_{t}^{B} \Gamma_{B}^{*} u \quad \text { for all } u \in D\left(\Gamma_{B}^{*}\right) .
\end{aligned}
$$

The subsequent lemma provides a square function estimate for the unperturbed Dirac-type operator $\Pi$. When considering square function estimates for the perturbed operator, there will be several instances where the perturbed case can be reduced with the assistance of this unperturbed estimate. Its proof follows directly from the self-adjointness of the operator $\Pi$ and Corollary 2.1 . 
Lemma 3.2 ([6, Lem. 4.6]) Suppose that the operators $\left\{\Gamma, B_{1}, B_{2}\right\}$ satisfy $(H 1)-(H 3)$. The quadratic estimate

$$
\int_{0}^{\infty}\left\|Q_{t} u\right\|^{2} \frac{d t}{t} \leq \frac{1}{2}\|u\|^{2}
$$

holds for all $u \in \mathcal{H}$. Equality holds on $\overline{R(\Pi)}$.

The following result will play a crucial role in the reduction of the square function estimate (3).

Proposition 3.3 ([6, Prop. 4.8]) Suppose that the operators $\left\{\Gamma, B_{1}, B_{2}\right\}$ satisfy $(H 1)-$ (H3). Assume that the estimate

$$
\int_{0}^{\infty}\left\|\Theta_{t}^{B} P_{t} u\right\|^{2} \frac{d t}{t} \leq c \cdot\|u\|^{2}
$$

holds for all $u \in R(\Gamma)$ and some constant $c>0$, together with three similar estimates obtained on replacing $\left\{\Gamma, B_{1}, B_{2}\right\}$ by $\left\{\Gamma^{*}, B_{2}, B_{1}\right\},\left\{\Gamma^{*}, B_{2}^{*}, B_{1}^{*}\right\}$ and $\left\{\Gamma, B_{1}^{*}, B_{2}^{*}\right\}$. Then $\Pi_{B}$ satisfies the quadratic estimate

$$
(c \cdot C)^{-1} \cdot\|u\|^{2} \leq \int_{0}^{\infty}\left\|Q_{t}^{B} u\right\|^{2} \frac{d t}{t} \leq c \cdot C \cdot\|u\|^{2}
$$

for all $u \in \overline{R\left(\Pi_{B}\right)}$, for some $C>0$ entirely dependent on $(H 1)-(H 3)$.

The constant dependence of (24) is not explicitly mentioned in Proposition 4.8 of [6], but it is relatively easy to trace through their argument and record where (23) is used. The following corollary is proved during the course of the proof of [6, Prop. 4.8].

Corollary 3.1 (High Frequency Estimate) Suppose that the operators $\left\{\Gamma, B_{1}, B_{2}\right\}$ satisfy (H1)-(H3). There exists a constant $c>0$ such that for any $u \in R(\Gamma)$,

$$
\int_{0}^{\infty}\left\|\Theta_{t}^{B}\left(I-P_{t}\right) u\right\|^{2} \frac{d t}{t} \leq c \cdot\|u\|^{2} .
$$

From this point onwards, it will also be assumed that our operators satisfy the additional hypotheses (H4)-(H6). These hypotheses are stated below for reference.

(H4) The Hilbert space is $\mathcal{H}=L^{2}\left(\mathbb{R}^{n} ; \mathbb{C}^{N}\right)$ for some $n, N \in \mathbb{N}^{*}$.

(H5) The operators $B_{1}$ and $B_{2}$ represent multiplication by matrix-valued functions. That is,

$$
B_{1}(f)(x)=B_{1}(x) \cdot f(x) \quad \text { and } \quad B_{2}(f)(x)=B_{2}(x) \cdot f(x)
$$

for all $f \in \mathcal{H}$ and $x \in \mathbb{R}^{n}$, where $B_{1}, B_{2} \in L^{\infty}\left(\mathbb{R}^{n} ; \mathcal{L}\left(\mathbb{C}^{N}\right)\right)$. 
(H6) For every bounded Lipschitz function $\eta: \mathbb{R}^{n} \rightarrow \mathbb{C}$, we have that $\eta D(\Gamma) \subset D(\Gamma)$ and $\eta D\left(\Gamma^{*}\right) \subset D\left(\Gamma^{*}\right)$. Moreover, the commutators $[\Gamma, \eta I]$ and $\left[\Gamma^{*}, \eta I\right]$ are multiplication operators that satisfy the bound

$$
|[\Gamma, \eta I](x)|,\left|\left[\Gamma^{*}, \eta I\right](x)\right| \leq c|\nabla \eta(x)|
$$

for all $x \in \mathbb{R}^{n}$ and some constant $c>0$.

In contrast to the original result, our operators will not be assumed to satisfy the cancellation condition (H7) or the coercivity condition (H8). Without these two conditions, many of the results from Sect. 5 of [6] will fail. One notable exception to this is that the bounded operators associated with our perturbed Dirac-type operator $\Pi_{B}$ will satisfy off-diagonal estimates.

Definition 3.2 (Off-Diagonal Bounds) Define $\langle x\rangle:=1+|x|$ for $x \in \mathbb{C}$ and $\operatorname{dist}(E, F):=\inf \{|x-y|: x \in E, y \in F\}$ for $E, F \subset \mathbb{R}^{n}$.

Let $\left\{U_{t}\right\}_{t>0}$ be a family of operators on $\mathcal{H}=L^{2}\left(\mathbb{R}^{n} ; \mathbb{C}^{N}\right)$. This collection is said to have off-diagonal bounds of order $M>0$ if there exists $C_{M}>0$ such that

$$
\left\|U_{t} u\right\|_{L^{2}(E)} \leq C_{M}\langle\operatorname{dist}(E, F) / t\rangle^{-M}\|u\|
$$

whenever $E, F \subset \mathbb{R}^{n}$ are Borel sets and $u \in \mathcal{H}$ satisfies supp $u \subset F$.

Proposition 3.4 ([6, Prop. 5.2]) Suppose that the operators $\left\{\Gamma, B_{1}, B_{2}\right\}$ satisfy (H1)(H6). Let $U_{t}$ be given by either $R_{t}^{B}, R_{-t}^{B}, P_{t}^{B}, Q_{t}^{B}$ or $\Theta_{t}^{B}$ for everyt $>0$. The collection of operators $\left\{U_{t}\right\}_{t>0}$ has off-diagonal bounds of every order $M>0$. Moreover, the constant $C_{M}$ in the estimate (25) depends only on $M$ and the hypotheses (H1)-(H6).

Introduce the following dyadic decomposition of $\mathbb{R}^{n}$. Let $\Delta=\cup_{j=-\infty}^{\infty} \Delta_{2^{j}}$ where $\Delta_{t}:=\left\{2^{j}\left(k+(0,1]^{n}\right): k \in \mathbb{Z}^{n}\right\}$ if $2^{j-1}<t \leq 2^{j}$ for $j \in \mathbb{Z}$. Define the averaging operator $A_{t}: \mathcal{H} \rightarrow \mathcal{H}$ through

$$
A_{t} u(x):=f_{Q(x, t)} u(y) d y:=\frac{1}{|Q(x, t)|} \int_{Q(x, t)} u(y) d y
$$

for $x \in \mathbb{R}^{n}, t>0$ and $u \in \mathcal{H}$, where $Q(x, t)$ is the unique dyadic cube in $\Delta_{t}$ that contains the point $x$.

For an operator family $\left\{U_{t}\right\}_{t>0}$ that satisfies off-diagonal bounds of every order, there exists an extension $U_{t}: L^{\infty}\left(\mathbb{R}^{n} ; \mathbb{C}^{N}\right) \rightarrow L_{\text {loc }}^{2}\left(\mathbb{R}^{n} ; \mathbb{C}^{N}\right)$ for each $t>0$. This is constructed by defining

$$
U_{t} u(x):=\lim _{r \rightarrow \infty} \sum_{\substack{R \in \Delta_{t} \\ \operatorname{dist}(Q, R)}} U_{t}\left(\mathbb{1}_{R} u\right)(x),
$$

for $x \in Q \in \Delta_{t}$ and $u \in L^{\infty}\left(\mathbb{R}^{n} ; \mathbb{C}^{N}\right)$. The convergence of the above limit is guaranteed by the off-diagonal bounds of $\left\{U_{t}\right\}_{t>0}$. Further detail on this construction can be found in $[6,9,10,18]$. The above extension then allows us to introduce the principal part of the operator $U_{t}$. 
Definition 3.3 Let $\left\{U_{t}\right\}_{t>0}$ be operators on $\mathcal{H}$ that satisfy off-diagonal bounds of every order. For $t>0$, the principal part of $U_{t}$ is the operator $\zeta_{t}: \mathbb{R}^{n} \rightarrow \mathcal{L}\left(\mathbb{C}^{N}\right)$ defined through

$$
\left[\zeta_{t}(x)\right](w):=\left(U_{t} w\right)(x)
$$

for each $x \in \mathbb{R}^{n}$ and $w \in \mathbb{C}^{N}$.

The following generalisation of Corollary 5.3 of [6] will also be true with an identical proof.

Proposition 3.5 Let $\left\{U_{t}\right\}_{t>0}$ be operators on $\mathcal{H}$ that satisfy off-diagonal bounds of every order. Let $\zeta_{t}: \mathbb{R}^{n} \rightarrow \mathcal{L}\left(\mathbb{C}^{N}\right)$ denote the principal part of the operator $U_{t}$. Then there exists $c>0$ such that

$$
f_{Q}\left|\zeta_{t}(y)\right|^{2} d y \leq c
$$

for all $Q \in \Delta_{t}$ and $t>0$. Moreover, the operators $\zeta_{t} A_{t}$ are uniformly $L^{2}$-bounded in $t$.

Finally, the ensuing partial result will also be valid. Its proof follows in an identical manner to the first part of the proof of Proposition 5.5 of [6].

Proposition 3.6 Let $\left\{U_{t}\right\}_{t>0}$ be operators on $\mathcal{H}$ that satisfy off-diagonal bounds of every order. Let $\zeta_{t}: \mathbb{R}^{n} \rightarrow \mathcal{L}\left(\mathbb{C}^{N}\right)$ denote the principal part of $U_{t}$. Then there exists $c>0$ such that

$$
\left\|\left(U_{t}-\zeta_{t} A_{t}\right) v\right\| \leq c \cdot\|t \nabla v\| .
$$

for any $v \in H^{1}\left(\mathbb{R}^{n} ; \mathbb{C}^{N}\right) \subset \mathcal{H}$ and $t>0$.

\subsection{Additional Structure}

At this point, further structure will be imposed upon our operators in order to generalise the non-homogeneous operator $\Gamma_{|V|^{\frac{1}{2}}}$ defined in (7). This additional structure will later be exploited in order to obtain square function estimates of the form (23).

Let $\mathbb{C}^{N}=V_{1} \oplus V_{2} \oplus V_{3}$ where $V_{1}, V_{2}$ and $V_{3}$ are finite-dimensional complex Hilbert spaces. Let $\mathbb{P}_{i}: \mathbb{C}^{N} \rightarrow \mathbb{C}^{N}$ be the projection operator onto the space $V_{i}$ for $i=1,2$ and 3. Our Hilbert space will have the following orthogonal decomposition

$$
\mathcal{H}:=L^{2}\left(\mathbb{R}^{n} ; \mathbb{C}^{N}\right)=L^{2}\left(\mathbb{R}^{n} ; V_{1}\right) \oplus L^{2}\left(\mathbb{R}^{n} ; V_{2}\right) \oplus L^{2}\left(\mathbb{R}^{n} ; V_{3}\right)
$$

The notation $\mathbb{P}_{i}$ will also be used to denote the natural projection operator from $\mathcal{H}$ onto $L^{2}\left(\mathbb{R}^{n} ; V_{i}\right)$. For a vector $v \in \mathcal{H}, v_{i} \in L^{2}\left(\mathbb{R}^{n} ; V_{i}\right)$ will denote the $i$ th component for $i=1,2$ or 3 . 
Let $\Gamma_{J}$ be an operator on $\mathcal{H}$ of the form

$$
\Gamma_{J}:=\left(\begin{array}{cc}
0 & 0 \\
D_{J} & 0
\end{array}\right):=\left(\begin{array}{ccc}
0 & 0 & 0 \\
J & 0 & 0 \\
D & 0 & 0
\end{array}\right),
$$

where $J$ and $D$ are closed densely defined operators

$$
\begin{aligned}
& J: L^{2}\left(\mathbb{R}^{n} ; V_{1}\right) \rightarrow L^{2}\left(\mathbb{R}^{n} ; V_{2}\right) \text { and } \\
& D: L^{2}\left(\mathbb{R}^{n} ; V_{1}\right) \rightarrow L^{2}\left(\mathbb{R}^{n} ; V_{3}\right),
\end{aligned}
$$

and $D_{J}: L^{2}\left(\mathbb{R}^{n} ; V_{1}\right) \rightarrow L^{2}\left(\mathbb{R}^{n} ; V_{2}\right) \oplus L^{2}\left(\mathbb{R}^{n} ; V_{3}\right)$ is the operator $D_{J}=\left(\begin{array}{c}J \\ D\end{array}\right)$ Define the operators

$$
\begin{aligned}
& \Gamma_{0}:=\left(\begin{array}{lll}
0 & 0 & 0 \\
0 & 0 & 0 \\
D & 0 & 0
\end{array}\right), \quad M_{J}:=\left(\begin{array}{lll}
0 & 0 & 0 \\
J & 0 & 0 \\
0 & 0 & 0
\end{array}\right), \\
& \Pi_{0}:=\Gamma_{0}+\Gamma_{0}^{*}, \quad S_{J}:=M_{J}+M_{J}^{*} \text { and } \Pi_{J}:=\Gamma_{J}+\Gamma_{J}^{*} .
\end{aligned}
$$

Let $B_{1}, B_{2} \in L^{\infty}\left(\mathbb{R}^{n} ; \mathcal{L}\left(\mathbb{C}^{N}\right)\right)$ be matrix-valued multiplication operators. The following key assumption will be imposed on our operators throughout the entirety of this article.

Key Assumption. The family of operators $\left\{\Gamma_{0}, B_{1}, B_{2}\right\}$ satisfies the conditions (H1)-(H8) of [6] while $\left\{\Gamma_{J}, B_{1}, B_{2}\right\}$ satisfies (H1)-(H6).

For reference, the cancellation condition (H7) and the coercivity condition (H8) are shown below for the operator $\Gamma_{0}$.

(H7) For any $u \in D\left(\Gamma_{0}\right)$ and $v \in D\left(\Gamma_{0}^{*}\right)$, both compactly supported,

$$
\int_{\mathbb{R}^{n}} \Gamma_{0} u=0 \text { and } \int_{\mathbb{R}^{n}} \Gamma_{0}^{*} v=0 .
$$

(H8) There exists $c>0$ such that

$$
\|\nabla u\| \leq c \cdot\left\|\Pi_{0} u\right\|
$$

for all $u \in R\left(\Pi_{0}\right) \cap D\left(\Pi_{0}\right)$.

Example 3.1 Typical examples of operators that satisfy the previous key assumption are when both $D$ and $J$ are partial differential operators of order less than or equal to one. If the perturbations $B_{1}$ and $B_{2}$ satisfy suitable accretivity conditions then the families of operators $\left\{\Gamma_{0}, B_{1}, B_{2}\right\}$ and $\left\{\Gamma_{J}, B_{1}, B_{2}\right\}$ will both satisfy (H1)-(H6). If, in addition, $D$ is first-order homogeneous and there exists $c>0$ for which

$$
\|\nabla u\| \leq c \cdot\|D u\|
$$


for all $u \in R\left(D^{*}\right) \cap D(D)$ and

$$
\|\nabla v\| \leq c \cdot\left\|D^{*} v\right\|
$$

for all $v \in R(D) \cap D\left(D^{*}\right)$ then $\left\{\Gamma_{0}, B_{1}, B_{2}\right\}$ will also satisfy (H7) and (H8). A particular example of such a situation is given by the operator $\Gamma_{|V|^{\frac{1}{2}}}$ together with perturbations $B_{1}$ and $B_{2}$ as defined in (7) and (8) with (1) and (6) satisfied.

Remark 3.2 Since the operator $\Gamma_{0}$, together with the perturbations $B_{1}$ and $B_{2}$, satisfy all eight conditions (H1)-(H8) of [6], it follows that any result from that paper must be valid for these operators.

Definition 3.4 For $t \in \mathbb{R} \backslash\{0\}$, define the perturbation dependent operators

$$
\begin{aligned}
\Gamma_{J, B} & :=B_{2}^{*} \Gamma_{J} B_{1}^{*}, \quad \Gamma_{J, B}^{*}:=B_{1} \Gamma_{J}^{*} B_{2}, \quad \Pi_{J, B}:=\Gamma_{J}+\Gamma_{J, B}^{*}, \\
R_{t}^{J, B} & :=\left(I+i t \Pi_{J, B}\right)^{-1}, \quad P_{t}^{J, B}:=\left(I+t^{2}\left(\Pi_{J, B}\right)^{2}\right)^{-1}, \\
Q_{t}^{J, B} & :=t \Pi_{J, B} P_{t}^{J, B} \text { and } \Theta_{t}^{J, B}:=t \Gamma_{J, B}^{*} P_{t}^{J, B} .
\end{aligned}
$$

When there is no perturbation, i.e. when $B_{1}=B_{2}=I$, the $B$ will dropped from the superscript or subscript. For example, instead of $\Theta_{t}^{J, I}$ the notation $\Theta_{t}^{J}$ will be employed.

We now introduce coercivity conditions to serve as a replacement for $(\mathrm{H} 8)$ for the operators $\left\{\Gamma_{J}, B_{1}, B_{2}\right\}$. These conditions will not be automatically imposed upon our operators but, rather, will be taken as hypotheses for our main results.

( $\mathrm{H} 8 \mathrm{D} \alpha)$ Let $\alpha \in(1,2]$. The domain inclusion

$$
D\left(\left(D_{J}^{*} D_{J}\right)^{\frac{\alpha}{2}}\right) \subset D\left(\left(D^{*} D\right)^{\frac{\alpha}{2}}\right)
$$

holds and there exists a constant $C>0$ such that

$$
\left\|\left(D^{*} D\right)^{\frac{\alpha}{2}} u\right\| \leq C \cdot\left\|\left(D_{J}^{*} D_{J}\right)^{\frac{\alpha}{2}} u\right\|
$$

for all $u \in D\left(\left(D_{J}^{*} D_{J}\right)^{\frac{\alpha}{2}}\right)$.

(H8J) $B_{2}$ is of the form

$$
B_{2}=\left(\begin{array}{cc}
I & 0 \\
0 & \hat{A}
\end{array}\right):=\left(\begin{array}{ccc}
I & 0 & 0 \\
0 & A_{22} & A_{23} \\
0 & A_{32} & A_{33}
\end{array}\right),
$$

where $A_{i j} \in L^{\infty}\left(\mathbb{R}^{n} ; \mathcal{L}\left(V_{j}, V_{i}\right)\right)$ for $i, j=2$ or 3 . The inclusion

$$
D\left(D_{J}^{*} D_{J}\right) \subset D\left(D_{J}^{*} \hat{A}\left(\begin{array}{l}
J \\
0
\end{array}\right)\right)
$$


is satisfied. Furthermore, there exists a constant $C>0$ such that for all $u \in$ $D\left(D_{J}^{*} D_{J}\right)$,

$$
\left\|D_{J}^{*} \hat{A}\left(\begin{array}{c}
J \\
0
\end{array}\right) u\right\| \leq C \cdot\left\|D_{J}^{*} D_{J} u\right\| .
$$

Remark 3.3 The situation of most interest to us is when $A_{32}=0$ and

$$
\left\|J^{*} A_{22} J u\right\|=\left\|J^{*} J u\right\|
$$

for all $u \in D\left(J^{*} A_{22} J\right)=D\left(J^{*} J\right)$. In this case, the domain inclusion of (H8J) becomes $D\left(D_{J}^{*} D_{J}\right) \subset D\left(J^{*} J\right)$ and the Riesz transform condition becomes the perturbation free condition

$$
\left\|J^{*} J u\right\| \leq C \cdot\left\|D_{J}^{*} D_{J} u\right\|
$$

for all $u \in D\left(D_{J}^{*} D_{J}\right)$. Furthermore, when this occurs, the Riesz transform condition of $(\mathrm{H} 8 \mathrm{~J})$ will be equivalent to the condition

$$
\left\|S_{J} u\right\| \leq C \cdot\left\|\Pi_{J} u\right\|
$$

or equivalently

$$
\left\|\Pi_{0} u\right\| \leq C \cdot\left\|\Pi_{J} u\right\|
$$

for all $u \in D\left(\Pi_{J}\right) \cap R\left(\Pi_{J}\right)$.

The Kato square root estimate is a first-order Riesz transform condition. To some extent, it then seems intuitively unnatural to use a second-order Riesz transform condition as in (H8J) as our hypotheses. Indeed, when the conditions of the above remark are satisfied and $J$ is a positive operator, it will be sufficient to consider a lower-order version of $(\mathrm{H} 8 \mathrm{~J})$ as given below.

( $\mathrm{H} 8 \mathrm{~J} \alpha)$ Let $\alpha \in(1,2]$. The perturbation $B_{2}$ is of the form (27) with $A_{32}=0 . J$ is a positive operator and

$$
\left\|J A_{22} J u\right\|=\left\|J^{2} u\right\|
$$

for all $u \in D\left(J A_{22} J\right)=D\left(J^{2}\right)$. The domain inclusion

$$
D\left(\left(D_{J}^{*} D_{J}\right)^{\frac{\alpha}{2}}\right) \subset D\left(J^{\alpha}\right)
$$

holds. Furthermore, there exists a constant $C>0$ such that

$$
\left\|J^{\alpha} u\right\| \leq C \cdot\left\|\left(D_{J}^{*} D_{J}\right)^{\frac{\alpha}{2}} u\right\|
$$




$$
\text { for all } u \in D\left(\left(D_{J}^{*} D_{J}\right)^{\frac{\alpha}{2}}\right) \text {. }
$$

Notation 1 For $\alpha \in(1,2]$, let $b_{\alpha}^{D}, b^{J}$ and $b_{\alpha}^{J}$ denote the smallest constant for which $(\mathrm{H} 8 \mathrm{D} \alpha),(\mathrm{H} 8 \mathrm{~J})$ or $(\mathrm{H} 8 \mathrm{~J} \alpha)$ are satisfied respectively. If the criteria for one of these conditions is not met then the corresponding constant will be set to infinity. For example, if $(\mathrm{H} 8 \mathrm{~J})$ is not satisfied then $b^{J}=\infty$. Also define

$$
c_{\alpha}^{J}:=\left(1+\left(b_{\alpha}^{D}\right)^{2}+\left(\min \left\{b^{J}, b_{\alpha}^{J}\right\}\right)^{2}\right) \cdot(\alpha-1)^{-1}
$$

For the remainder of this article we introduce the notation $A \lesssim B$ and $A \simeq B$ to denote that there exists a constant $C>0$, independent of $(\mathrm{H} 8 \mathrm{D} \alpha),(\mathrm{H} 8 \mathrm{~J})$ and $(\mathrm{H} 8 \mathrm{~J} \alpha)$, for which $A \leq C \cdot B$ and $C^{-1} \cdot B \leq A \leq C \cdot B$ respectively. $C$ is still allowed to depend on (H1)-(H8) for $\left\{\Gamma_{0}, B_{1}, B_{2}\right\}$ and (H1)-(H6) for $\left\{\Gamma_{J}, B_{1}, B_{2}\right\}$. Note that this implies that $C$ will only depend on $J$ through the constants in (H2) for $\left\{\Gamma_{J}, B_{1}, B_{2}\right\}$ and it will be independent of $\alpha$.

We are now in a position where the main result of the non-homogeneous AKM framework can finally be stated.

Theorem 3.1 Let $\left\{\Gamma_{J}, B_{1}, B_{2}\right\}$ be as defined above. Consider the following square function estimate:

$$
\int_{0}^{\infty}\left\|\Theta_{t}^{J, B} \mathbb{P}_{i} P_{t}^{J} u\right\|^{2} \frac{d t}{t} \lesssim C \cdot\|u\|^{2}
$$

for all $u \in R\left(\Gamma_{J}\right)$, for some $C>0$, for $i=1,2$ or 3 .

(i) The estimate is trivially satisfied for $i=1$ for any $C \geq 0$.

(ii) If (H8J) is satisfied then (28) is satisfied for $i=2$ with $C=\left(b^{J}\right)^{2}$.

(iii) If $(H 8 J \alpha)$ is satisfied for some $\alpha \in(1,2]$ then (28) is satisfied for $i=2$ with $C=\left(1+\left(b_{\alpha}^{J}\right)^{2}\right)(\alpha-1)^{-1}$.

(iv) If $(H 8 D \alpha)$ is satisfied for some $\alpha \in(1,2]$ and either $(H 8 J)$ or $(H 8 J \alpha)$ is also satisfied then (28) holds for $i=3$ with constant $C=c_{\alpha}^{J}$.

Proof The proof of (iii) and (iv) will be postponed until Sect. 4. For (i), simply note that since $\Gamma_{J}$ commutes with the operator $P_{t}^{J}$ by Lemma 3.1 we must have $\mathbb{P}_{1} P_{t}^{J} u=0$ for any $u \in R\left(\Gamma_{J}\right)$.

It remains to consider (ii). Suppose that $(\mathrm{H} 8 \mathrm{~J})$ holds. First it will be proved that for $u \in R\left(\Gamma_{J}\right)$ we have $\mathbb{P}_{2} P_{t}^{J} u \in D\left(\Gamma_{J, B}^{*}\right)$. Since $u \in R\left(\Gamma_{J}\right), u=\Gamma_{J} v$ for some $v \in D\left(\Gamma_{J}\right)$. As

$$
P_{t}^{J} u=P_{t}^{J} \Gamma_{J} v=\Gamma_{J} P_{t}^{J} v
$$


by Lemma 3.1 and $P_{t}^{J} u \in D\left(\Pi_{J}\right)$, it follows that $\left(P_{t}^{J} v\right)_{1} \in D\left(D_{J}^{*} D_{J}\right)$, which by (H8J) is contained in $D\left(D_{J}^{*} \hat{A}\left(\begin{array}{l}J \\ 0\end{array}\right)\right)$. This implies that

$$
\left(\begin{array}{c}
J\left(P_{t}^{J} v\right)_{1} \\
0
\end{array}\right)=\left(\begin{array}{c}
\left(P_{t}^{J} u\right)_{2} \\
0
\end{array}\right) \in D\left(D_{J}^{*} \hat{A}\right)
$$

and therefore $\mathbb{P}_{2} P_{t}^{J} u \in D\left(\Gamma_{J, B}^{*}\right)$.

Since $\mathbb{P}_{2} P_{t}^{J} u \in D\left(\Gamma_{J, B}^{*}\right)$, it follows from Lemma 3.1 that

$$
\Theta_{t}^{J, B} \mathbb{P}_{2} P_{t}^{J} u=P_{t}^{J, B} t \Gamma_{J, B}^{*} \mathbb{P}_{2} P_{t}^{J} u
$$

The estimate in (H8J) gives

$$
\left\|\Gamma_{J, B}^{*} \mathbb{P}_{2} \tilde{v}\right\| \leq b^{J} \cdot\left\|\Gamma_{J}^{*} \tilde{v}\right\|
$$

for any $\tilde{v} \in R\left(\Gamma_{J}\right) \cap D\left(\Pi_{J}\right)$. Since $P_{t}^{J}$ and $\Gamma_{J}$ commute by Lemma 3.1, it follows that

$$
\left\|\Gamma_{J, B}^{*} \mathbb{P}_{2} P_{t}^{J} u\right\| \leq b^{J} \cdot\left\|\Gamma_{J}^{*} P_{t}^{J} u\right\|
$$

for $u \in R\left(\Gamma_{J}\right)$. On applying the uniform $L^{2}$-boundedness of the $P_{t}^{J, B}$ operators,

$$
\begin{aligned}
\int_{0}^{\infty}\left\|\Theta_{t}^{J, B} \mathbb{P}_{2} P_{t}^{J} u\right\|^{2} \frac{d t}{t} & =\int_{0}^{\infty}\left\|P_{t}^{J, B} t \Gamma_{J, B}^{*} \mathbb{P}_{2} P_{t}^{J} u\right\|^{2} \frac{d t}{t} \\
& \lesssim \int_{0}^{\infty}\left\|t \Gamma_{J, B}^{*} \mathbb{P}_{2} P_{t}^{J} u\right\|^{2} \frac{d t}{t} \\
& \leq\left(b^{J}\right)^{2} \int_{0}^{\infty}\left\|t \Gamma_{J}^{*} P_{t}^{J} u\right\|^{2} \frac{d t}{t}
\end{aligned}
$$

On successively applying Proposition 3.1 and Lemma 3.2 we obtain

$$
\begin{aligned}
\int_{0}^{\infty}\left\|\Theta_{t}^{J, B} \mathbb{P}_{2} P_{t}^{J} u\right\|^{2} \frac{d t}{t} & \lesssim\left(b^{J}\right)^{2} \int_{0}^{\infty}\left\|t \Pi_{J} P_{t}^{J} u\right\|^{2} \frac{d t}{t} \\
& =\left(b^{J}\right)^{2} \int_{0}^{\infty}\left\|Q_{t}^{J} u\right\|^{2} \frac{d t}{t} \\
& =\frac{1}{2}\left(b^{J}\right)^{2}\|u\|^{2}
\end{aligned}
$$

This shows that (28) is valid for $i=2$ with constant $C=\left(b^{J}\right)^{2}$.

Let's consider an estimate that serves as a dual to (28). 
Proposition 3.7 For $t>0$, define the operator

$$
\underline{P}_{t}^{J, B}:=\left(I+t^{2}\left(\Gamma_{J}^{*}+B_{2} \Gamma_{J} B_{1}\right)^{2}\right)^{-1} .
$$

The square function estimate

$$
\int_{0}^{\infty}\left\|t B_{2} \Gamma_{J} B_{1} \underline{P}_{t}^{J, B} P_{t}^{J} u\right\|^{2} \frac{d t}{t} \lesssim\|u\|^{2}
$$

will hold for all $u \in \mathcal{H}$ when $B_{1}=I$.

Proof Since $\left\{\Gamma_{J}, B_{1}, B_{2}\right\}$ satisfies (H1)-(H6) it follows that $\left\{\Gamma_{J}^{*}, B_{2}, B_{1}\right\}$ must also satisfy (H1)-(H6). Proposition 3.2 then implies that the operators $\underline{P}_{t}^{J, B}$ are welldefined and uniformly $L^{2}$-bounded. Since $B_{1}=I$, it follows that $P_{t}^{J} u \in D\left(B_{2} \Gamma_{J} B_{1}\right)$ for any $u \in \mathcal{H}$ and therefore, by Lemma 3.1,

$$
B_{2} \Gamma_{J} B_{1} \underline{P}_{t}^{J, B} P_{t}^{J} u=\underline{P}_{t}^{J, B} B_{2} \Gamma_{J} B_{1} P_{t}^{J} u=\underline{P}_{t}^{J} B_{2} \Gamma_{J} P_{t}^{J} u .
$$

This together with the uniform $L^{2}$-boundedness of the $\underline{P}_{t}^{J, B}$ operators implies that

$$
\begin{aligned}
\int_{0}^{\infty}\left\|t B_{2} \Gamma_{J} B_{1} \underline{P}_{t}^{J, B} P_{t}^{J} u\right\|^{2} \frac{d t}{t} & =\int_{0}^{\infty}\left\|\underline{P}_{t}^{J, B} t B_{2} \Gamma_{J} P_{t}^{J} u\right\|^{2} \frac{d t}{t} \\
& \lesssim \int_{0}^{\infty}\left\|t \Gamma_{J} P_{t}^{J} u\right\|^{2} \frac{d t}{t} \\
& \leq \int_{0}^{\infty}\left\|t \Pi_{J} P_{t}^{J} u\right\|^{2} \frac{d t}{t} \\
& =\int_{0}^{\infty}\left\|Q_{t}^{J} u\right\|^{2} \frac{d t}{t} \\
& =\frac{1}{2}\|u\|^{2}
\end{aligned}
$$

where the inequality $\left\|\Gamma_{J} v\right\| \leq\left\|\Pi_{J} v\right\|$ for $v \in D\left(\Pi_{J}\right)$ follows immediately from the three-by-three matrix form of the operators and Lemma 3.2 was applied to obtain the last line.

From our main result, Theorem 3.1, and the previous proposition, the upper and lower square function estimates for $Q_{t}^{J, B}$ can be proved.

Theorem 3.2 Suppose that $B_{1}=I$. Suppose further that $(H 8 D \alpha)$ is satisfied for some $\alpha \in(1,2]$ and either $(H 8 J)$ or $(H 8 J \alpha)$ is satisfied. Then

$$
\left(c_{\alpha}^{J}\right)^{-1} \cdot\|u\|^{2} \lesssim \int_{0}^{\infty}\left\|Q_{t}^{J, B} u\right\|^{2} \frac{d t}{t} \lesssim c_{\alpha}^{J} \cdot\|u\|^{2}
$$

for all $u \in \overline{R\left(\Pi_{J}\right)}$. 
Proof Proposition 3.3 states that in order to prove the square function estimate (30), it is sufficient for the estimate (28) to be valid for all $i=1,2$ and 3 for the permutations of operators $\left\{\Gamma_{J}, B_{1}, B_{2}\right\},\left\{\Gamma_{J}, B_{1}^{*}, B_{2}^{*}\right\},\left\{\Gamma_{J}^{*}, B_{2}, B_{1}\right\}$ and $\left\{\Gamma_{J}^{*}, B_{2}^{*}, B_{1}^{*}\right\}$. The permutations $\left\{\Gamma_{J}, B_{1}, B_{2}\right\}$ and $\left\{\Gamma_{J}, B_{1}^{*}, B_{2}^{*}\right\}$ both come under the umbrella of Theorem 3.1 and the permutations $\left\{\Gamma_{J}^{*}, B_{2}, B_{1}\right\}$ and $\left\{\Gamma_{J}^{*}, B_{2}^{*}, B_{1}^{*}\right\}$ are handled by Proposition 3.7 .

From the upper and lower estimate of the previous theorem, Theorem 2.4 then implies that $\Pi_{J, B}$ has a bounded holomorphic functional calculus.

Theorem 3.3 Suppose that $B_{1}=I$. Suppose further that $(H 8 D \alpha)$ is satisfied for some $\alpha \in(1,2]$ and either $(H 8 J)$ or $(H 8 J \alpha)$ is satisfied. Then $\Pi_{J, B}$ has a bounded $H^{\infty}\left(S_{\mu}^{o}\right)$-holomorphic functional calculus for any $\mu \in\left(\omega_{J}, \frac{\pi}{2}\right)$, where

$$
\omega_{J}:=\frac{1}{2}\left(\sup _{u \in R\left(\Gamma_{J}^{*}\right) \backslash\{0\}}\left|\arg \left\langle B_{1} u, u\right\rangle\right|+\sup _{u \in R\left(\Gamma_{J}\right) \backslash\{0\}}\left|\arg \left\langle B_{2} u, u\right\rangle\right|\right) .
$$

In particular,

$$
\left\|f\left(\Pi_{J, B}\right)\right\| \lesssim c_{\alpha}^{J} \cdot \sup _{\zeta \in S_{\mu}^{o}}|f(\zeta)|
$$

for any $f \in H_{0}^{\infty}\left(S_{\mu}^{o}\right)$.

Corollary 3.2 Suppose that $B_{1}=I$. Suppose further that $(H 8 D \alpha)$ is satisfied for some $\alpha \in(1,2]$ and either $(H 8 J)$ or $(H 8 J \alpha)$ is satisfied. The operator

$$
L_{B}^{J}:=D_{J}^{*} \hat{A} D_{J}
$$

is a $2 \omega_{J}$-sectorial operator with a bounded $H^{\infty}\left(S_{2 \mu+}^{o}\right)$-functional calculus for any $\mu \in\left(\omega_{J}, \frac{\pi}{2}\right)$ and

$$
\left\|\sqrt{L_{B}^{J}} u\right\| \simeq c_{\alpha}^{J} \cdot(\|J u\|+\|D u\|)
$$

for all $u \in D(J) \cap D(D)$.

Proof The bounded $H^{\infty}\left(S_{2 \mu+}^{o}\right)$-functional calculus of $L_{B}^{J}$ follows from the bounded $H^{\infty}\left(S_{\mu}^{o}\right)$-functional calculus of $\Pi_{J, B}$ and that $\Pi_{J, B}^{2}$ is of the form

$$
\Pi_{J, B}^{2}=\left(\begin{array}{ccc}
L_{B}^{J} & 0 & 0 \\
0 & * & * \\
0 & * & *
\end{array}\right)
$$

The estimate (31) follows from Corollary 2.2 applied to the operator $\Pi_{J, B}$ and an element $(u, 0,0) \in \mathcal{H}$ with $u \in D(J) \cap D(D)$. 


\section{Square Function Estimates}

In this section, a proof of our main result, Theorem 3.1, will be provided. The first part of the proof consists in showing that the operators $P_{t}^{J}$ can effectively be diagonalised when estimating square function norms from above. This diagonalisation will be applied to bound the second component of our square function norm when $(\mathrm{H} 8 \mathrm{~J} \alpha)$ is satisfied thus proving the third part of Theorem 3.1. To prove the fourth and most challenging part of Theorem 3.1 we will use this diagonalisation and an argument similar to the original result [6]. That is, a $T(1)$-type reduction will be applied to reduce the third component of the square function norm to a Carleson measure norm which will subsequently be proved to be bounded.

\subsection{Diagonalisation of the $P_{t}^{J}$ Operators}

Define the bounded operator $\mathcal{P}_{t}^{J}: \mathcal{H} \rightarrow \mathcal{H}$ through

$$
\mathcal{P}_{t}^{J}:=\left(\begin{array}{ccc}
\left(I+t^{2} D_{J}^{*} D_{J}\right)^{-1} & 0 & 0 \\
0 & \left(I+t^{2} J J^{*}\right)^{-1} & 0 \\
0 & 0 & \left(I+t^{2} D D^{*}\right)^{-1}
\end{array}\right)
$$

for $t>0$. Observe that since the operators $D_{J}^{*} D_{J}, J J^{*}$ and $D D^{*}$ are all self-adjoint, it follows from Corollary 2.1 that square function estimates hold for each of these operators with constant independent of $J$ and $D$. Therefore each of these operators possesses a bounded holomorphic functional calculus with constant independent of $J$ and $D$. It can be deduced from this that the operators $\mathcal{P}_{t}^{J}$ are uniformly $L^{2}$-bounded with constant independent of $J$ and $D$.

Let us prove that the operator $P_{t}^{J}$ can be effectively diagonalised when evaluating square function estimates. Specifically, the following theorem will be proved.

Theorem 4.1 Suppose that $(H 8 D \alpha)$ is satisfied for some $\alpha \in(1,2]$. Then

$$
\int_{0}^{\infty}\left\|\mathbb{P}_{3}\left(\mathcal{P}_{t}^{J}-P_{t}^{J}\right) u\right\|^{2} \frac{d t}{t} \lesssim\left(1+\left(b_{\alpha}^{D}\right)^{2}\right) \cdot\|u\|^{2}
$$

for all $u \in R\left(\Gamma_{J}\right)$. Suppose, in addition, that (H8J $\left.\alpha\right)$ is also satisfied. Then

$$
\int_{0}^{\infty}\left\|\left(\mathcal{P}_{t}^{J}-P_{t}^{J}\right) u\right\|^{2} \frac{d t}{t} \lesssim c_{\alpha}^{J} \cdot\|u\|^{2}
$$

for all $u \in R\left(\Gamma_{J}\right)$.

Such a diagonalisation will aid us tremendously in the bounding of our main square function estimate (28) for the second and third component. This theorem will be proved by inspecting each component separately.

Remark 4.1 It is easy to see that the diagonalisation estimate (32) is trivially satisfied on the first component for any $u \in \mathcal{H}$ since $\mathbb{P}_{1} \mathcal{P}_{t}^{J}=\mathbb{P}_{1} P_{t}^{J}$. 
Proposition 4.1 For any $u \in \mathcal{H}$,

$$
\int_{0}^{\infty}\left\|\mathcal{P}_{t}^{J}\left(P_{t}^{J}-I\right) u\right\|^{2} \frac{d t}{t} \lesssim\|u\|^{2} .
$$

Proof The estimate is trivially satisfied for any $u \in N\left(\Pi_{J}\right)$ since

$$
\begin{aligned}
\left(P_{t}^{J}-I\right) u & =\left(\left(I+t^{2} \Pi_{J}^{2}\right)^{-1}-I\right) u \\
& =\left(I+t^{2} \Pi_{J}^{2}\right)^{-1}\left(I-\left(I+t^{2} \Pi_{J}^{2}\right)\right) u \\
& =0
\end{aligned}
$$

for any $t>0$. Suppose that $u \in \overline{R\left(\Pi_{J}\right)}$. On applying the resolution of the identity, Proposition 2.3,

$$
\begin{aligned}
\int_{0}^{\infty}\left\|\mathcal{P}_{t}^{J}\left(P_{t}^{J}-I\right) u\right\|^{2} \frac{d t}{t} & =\int_{0}^{\infty}\left\|\mathcal{P}_{t}^{J}\left(P_{t}^{J}-I\right) 2 \int_{0}^{\infty}\left(Q_{s}^{J}\right)^{2} u \frac{d s}{s}\right\|^{2} \frac{d t}{t} \\
& \lesssim \int_{0}^{\infty}\left(\int_{0}^{\infty}\left\|\mathcal{P}_{t}^{J}\left(P_{t}^{J}-I\right)\left(Q_{s}^{J}\right)^{2} u\right\| \frac{d s}{s}\right)^{2} \frac{d t}{t}
\end{aligned}
$$

The Cauchy-Schwarz inequality leads to

$$
\begin{aligned}
& \int_{0}^{\infty}\left\|\mathcal{P}_{t}^{J}\left(P_{t}^{J}-I\right) u\right\|^{2} \frac{d t}{t} \\
& \quad \lesssim \int_{0}^{\infty}\left(\int_{0}^{\infty}\left\|\mathcal{P}_{t}^{J}\left(P_{t}^{J}-I\right) Q_{s}^{J}\right\| \frac{d s}{s}\right) \\
&\left(\int_{0}^{\infty}\left\|\mathcal{P}_{t}^{J}\left(P_{t}^{J}-I\right) Q_{s}^{J}\right\|\left\|Q_{s}^{J} u\right\|^{2} \frac{d s}{s}\right) \frac{d t}{t}
\end{aligned}
$$

Let's estimate the term $\left\|\mathcal{P}_{t}^{J}\left(P_{t}^{J}-I\right) Q_{s}^{J}\right\|$. First assume that $t \leq s$. On noting that $\left(P_{t}^{J}-I\right) Q_{s}^{J}=\frac{t}{s} Q_{t}^{J}\left(P_{s}^{J}-I\right)$ we obtain

$$
\left\|\mathcal{P}_{t}^{J}\left(P_{t}^{J}-I\right) Q_{s}^{J}\right\| \lesssim\left\|\left(P_{t}^{J}-I\right) Q_{s}^{J}\right\| \lesssim \frac{t}{s}\left\|Q_{t}^{J}\left(P_{s}^{J}-I\right)\right\| \lesssim \frac{t}{s}
$$

Next, suppose that $t>s$. Then the equality $P_{t}^{J} Q_{s}^{J}=\frac{s}{t} Q_{t}^{J} P_{s}^{J}$ gives

$$
\left\|\mathcal{P}_{t}^{J}\left(P_{t}^{J}-I\right) Q_{s}^{J}\right\| \lesssim\left\|P_{t}^{J} Q_{s}^{J}\right\|+\left\|\mathcal{P}_{t}^{J} Q_{s}^{J}\right\| \lesssim \frac{s}{t}+\left\|\mathcal{P}_{t}^{J} Q_{s}^{J}\right\|
$$

The term $\mathcal{P}_{t}^{J} Q_{s}^{J}$ will be considered component-wise. For the first component, recall that $\mathbb{P}_{1} \mathcal{P}_{t}^{J}=\mathbb{P}_{1} P_{t}^{J}$ and observe that

$$
\left\|\mathbb{P}_{1} \mathcal{P}_{t}^{J} Q_{s}^{J}\right\|=\left\|\mathbb{P}_{1} P_{t}^{J}{ }_{s} \Pi_{J} P_{s}^{J}\right\|
$$




$$
\begin{aligned}
& =\frac{s}{t}\left\|\mathbb{P}_{1} P_{t}^{J} t \Pi_{J} P_{s}^{J}\right\| \\
& =\frac{s}{t}\left\|\mathbb{P}_{1} Q_{t}^{J} P_{s}^{J}\right\| \\
& \lesssim \frac{s}{t} .
\end{aligned}
$$

For the second component, note that

$$
\mathbb{P}_{2} \mathcal{P}_{t}^{J}=\left(I+t^{2} S_{J}^{2}\right)^{-1} \mathbb{P}_{2}
$$

Also observe

$$
\mathbb{P}_{2} \Pi_{J} u=\mathbb{P}_{2} \Pi_{J} \mathbb{P}_{1} u=\mathbb{P}_{2} S_{J} \mathbb{P}_{1} u
$$

for $u \in D\left(\Pi_{J}\right)$. This gives

$$
\begin{aligned}
\left\|\mathbb{P}_{2} \mathcal{P}_{t}^{J} Q_{s}^{J}\right\| & =\left\|\left(I+t^{2} S_{J}^{2}\right)^{-1} \mathbb{P}_{2} s \Pi_{J} P_{s}^{J}\right\| \\
& =\left\|\left(I+t^{2} S_{J}^{2}\right)^{-1} \mathbb{P}_{2} s S_{J} \mathbb{P}_{1} P_{s}^{J}\right\| \\
& =\frac{s}{t}\left\|\mathbb{P}_{2} t S_{J}\left(I+t^{2} S_{J}^{2}\right)^{-1} \mathbb{P}_{1} P_{s}^{J}\right\| \\
& \lesssim \frac{s}{t},
\end{aligned}
$$

where the last line follows from the fact that $S_{J}$ is self-adjoint and therefore possesses a bounded holomorphic functional calculus with constant independent of $J$. Lastly, for the third component, we have

$$
\mathbb{P}_{3} \mathcal{P}_{t}^{J}=\mathbb{P}_{3} P_{t}^{0}=P_{t}^{0} \mathbb{P}_{3}
$$

and

$$
\mathbb{P}_{3} \Pi_{J} u=\mathbb{P}_{3} \Pi_{J} \mathbb{P}_{1} u=\mathbb{P}_{3} \Pi_{0} \mathbb{P}_{1} u
$$

for $u \in D\left(\Pi_{J}\right)$. This leads to

$$
\begin{aligned}
\left\|\mathbb{P}_{3} \mathcal{P}_{t}^{J} Q_{s}^{J}\right\| & =\left\|P_{t}^{0} \mathbb{P}_{3} s \Pi_{J} P_{s}^{J}\right\| \\
& =\left\|P_{t}^{0} \mathbb{P}_{3} s \Pi_{0} \mathbb{P}_{1} P_{s}^{J}\right\| \\
& =\frac{s}{t}\left\|\mathbb{P}_{3} t \Pi_{0} P_{t}^{0} \mathbb{P}_{1} P_{s}^{J}\right\| \\
& \lesssim \frac{s}{t} .
\end{aligned}
$$


Putting everything together gives

$$
\left\|\mathcal{P}_{t}^{J}\left(P_{t}^{J}-I\right) Q_{s}^{J}\right\| \lesssim \min \left\{\frac{t}{s}, \frac{s}{t}\right\} .
$$

This bound can then be applied to (34) to give (33).

Proposition 4.2 Suppose that the condition $(H 8 J \alpha)$ is satisfied for some $\alpha \in(1,2]$. Then

$$
\int_{0}^{\infty}\left\|\mathbb{P}_{2}\left(I-\mathcal{P}_{t}^{J}\right) P_{t}^{J} u\right\|^{2} \frac{d t}{t} \lesssim(\alpha-1)^{-1}\left(b_{\alpha}^{J}\right)^{2} \cdot\|u\|^{2}
$$

for any $u \in R\left(\Gamma_{J}\right)$.

Proof It will first be proved that $\left(P_{t}^{J} u\right)_{2} \in D\left(J^{\alpha-1}\right)$. Since $\Gamma_{J}$ commutes with $P_{t}^{J}$ we must have

$$
P_{t}^{J} u=\Gamma_{J}\left(\begin{array}{l}
v \\
0 \\
0
\end{array}\right)
$$

for some $(v, 0,0) \in D\left(\Gamma_{J}\right)$. This then gives $\left(P_{t}^{J} u\right)_{2}=J v$. Therefore $\left(P_{t}^{J} u\right)_{2} \in$ $D\left(J^{\alpha-1}\right)$ if and only if $v \in D\left(J^{\alpha}\right)$. We know that $P_{t}^{J} u \in D\left(\Pi_{J}\right)$ which implies that $v \in D\left(D_{J}^{*} D_{J}\right)$ and therefore $v \in D\left(\left(D_{J}^{*} D_{J}\right)^{\frac{\alpha}{2}}\right)$. Our hypothesis $(\mathrm{H} 8 \mathrm{~J} \alpha)$ then tells us that $v \in D\left(J^{\alpha}\right)$ which allows us to conclude, using the previous reasoning, that $\left(P_{t}^{J} u\right)_{2} \in D\left(J^{\alpha-1}\right)$.

Since $\left(P_{t}^{J} u\right)_{2} \in D\left(J^{\alpha-1}\right)$, it follows that

$$
\begin{aligned}
\mathbb{P}_{2}\left(I-\mathcal{P}_{t}^{J}\right) P_{t}^{J} u & =\left(0, t^{2} J^{2}\left(I+t^{2} J^{2}\right)^{-1}\left(P_{t}^{J} u\right)_{2}, 0\right) \\
& =\left(0, g_{\alpha}^{t}(J) t^{\alpha-1} J^{\alpha-1}\left(P_{t}^{J} u\right)_{2}, 0\right)
\end{aligned}
$$

where $g_{\alpha}^{t}: S_{\mu}^{o} \rightarrow \mathbb{C}$ is the bounded holomorphic function defined through

$$
g_{\alpha}^{t}(z):=\frac{t^{2} z^{2}}{\left(I+t^{2} z^{2}\right) t^{\alpha-1}\left(\sqrt{z^{2}}\right)^{\alpha-1}} .
$$

As $J$ is self-adjoint, it follows from Corollary 2.1 that $J$ possesses a bounded holomorphic functional calculus with constant independent of $J$. This, together with (37), gives

$$
\left\|\mathbb{P}_{2}\left(I-\mathcal{P}_{t}^{J}\right) P_{t}^{J} u\right\| \lesssim\left\|t^{\alpha-1} J^{\alpha-1}\left(P_{t}^{J} u\right)_{2}\right\|
$$


On applying $(\mathrm{H} 8 \mathrm{~J} \alpha)$,

$$
\begin{aligned}
\left\|J^{\alpha-1}\left(P_{t}^{J} u\right)_{2}\right\| & =\left\|J^{\alpha} v\right\| \\
& \leq b_{\alpha}^{J} \cdot\left\|\left(D_{J}^{*} D_{J}\right)^{\frac{\alpha}{2}} v\right\| \\
& =b_{\alpha}^{J} \cdot\left\|\left|\Pi_{J}\right|^{\alpha}(v, 0,0)\right\| \\
& \simeq b_{\alpha}^{J} \cdot\left\|\left|\Pi_{J}\right|^{\alpha-1} \Pi_{J}(v, 0,0)\right\| \\
& =b_{\alpha}^{J} \cdot\left\|\left|\Pi_{J}\right|^{\alpha-1} P_{t}^{J} u\right\|,
\end{aligned}
$$

where $\left|\Pi_{J}\right|:=\sqrt{\Pi_{J}^{2}}$ and in the fourth line we applied the bounded holomorphic functional calculus of the operator $\Pi_{J}$. Therefore

$$
\begin{aligned}
\int_{0}^{\infty}\left\|\mathbb{P}_{2}\left(I-\mathcal{P}_{t}^{J}\right) P_{t}^{J} u\right\|^{2} \frac{d t}{t} & \lesssim\left(b_{\alpha}^{J}\right)^{2} \cdot \int_{0}^{\infty}\left\|t^{\alpha-1}\left|\Pi_{J}\right|^{\alpha-1} P_{t}^{J} u\right\|^{2} \frac{d t}{t} \\
& \lesssim(\alpha-1)^{-1}\left(b_{\alpha}^{J}\right)^{2} \cdot\|u\|^{2}
\end{aligned}
$$

where we used the fact that $\Pi_{J}$ is self-adjoint and Corollary 2.1 in the last line.

Proposition 4.3 Suppose that $(H 8 D \alpha)$ is satisfied for some $\alpha \in(1,2]$. Then

$$
\int_{0}^{\infty}\left\|\mathbb{P}_{3}\left(I-\mathcal{P}_{t}^{J}\right) P_{t}^{J} u\right\|^{2} \frac{d t}{t} \lesssim(\alpha-1)^{-1}\left(b_{\alpha}^{D}\right)^{2} \cdot\|u\|^{2}
$$

for all $u \in R\left(\Gamma_{J}\right)$.

Proof First note that the left-hand side of (39) can be re-written as

$$
\begin{aligned}
\int_{0}^{\infty}\left\|\mathbb{P}_{3}\left(I-\mathcal{P}_{t}^{J}\right) P_{t}^{J} u\right\|^{2} \frac{d t}{t} & =\int_{0}^{\infty}\left\|\mathbb{P}_{3}\left(I-P_{t}^{0}\right) P_{t}^{J} u\right\|^{2} \frac{d t}{t} \\
& =\int_{0}^{\infty}\left\|\left(I-P_{t}^{0}\right) \mathbb{P}_{3} P_{t}^{J} u\right\|^{2} \frac{d t}{t} .
\end{aligned}
$$

It will be shown that $\mathbb{P}_{3} P_{t}^{J} u \in D\left(\left|\Pi_{0}\right|^{\alpha-1}\right)$. Since $\Gamma_{J}$ commutes with the operator $P_{t}^{J}$ and $u \in R\left(\Gamma_{J}\right)$, we must have $P_{t}^{J} u=\Gamma_{J} P_{t}^{J}(v, 0,0)$ for some $(v, 0,0) \in D\left(\Gamma_{J}\right)$. This implies that

$$
\mathbb{P}_{3} P_{t}^{J} u=\mathbb{P}_{3} \Gamma_{J} P_{t}^{J}(v, 0,0)=\mathbb{P}_{3} \Pi_{0} P_{t}^{J}(v, 0,0)
$$

and therefore $\mathbb{P}_{3} P_{t}^{J} u \in D\left(\left|\Pi_{0}\right|^{\alpha-1}\right)$ will follow from $P_{t}^{J}(v, 0,0) \in D\left(\left|\Pi_{0}\right|^{\alpha-1} \mathbb{P}_{3} \Pi_{0}\right)$ which itself will follow from $P_{t}^{J}(v, 0,0) \in D\left(\left|\Pi_{0}\right|^{\alpha-1} \Pi_{0}\right)$. The bounded holomorphic functional calculus of the operator $\Pi_{0}$ tells us that $D\left(\left|\Pi_{0}\right|^{\alpha-1} \Pi_{0}\right)=D\left(\left|\Pi_{0}\right|^{\alpha}\right)$ 
and it is therefore sufficient to prove that $P_{t}^{J}(v, 0,0) \in D\left(\left|\Pi_{0}\right|^{\alpha}\right)$. Since $P_{t}^{J}(v, 0,0)$ is non-zero only in the first component, this in turn is equivalent to proving that

$$
\left(P_{t}^{J}(v, 0,0)\right)_{1} \in D\left(\left(D^{*} D\right)^{\frac{\alpha}{2}}\right) .
$$

This however follows directly from our hypothesis $(\mathrm{H} 8 \mathrm{D} \alpha)$ and the fact that $\left(P_{t}^{J}(v, 0,0)\right)_{1} \in D\left(D_{J}^{*} D_{J}\right) \subset D\left(\left(D_{J}^{*} D_{J}\right)^{\frac{\alpha}{2}}\right)$. This completes the proof of our claim that $\mathbb{P}_{3} P_{t}^{J} u \in D\left(\left|\Pi_{0}\right|^{\alpha-1}\right)$.

Since $\mathbb{P}_{3} P_{t}^{J} u \in D\left(\left|\Pi_{0}\right|^{\alpha-1}\right)$ we must have

$$
\left(I-P_{t}^{0}\right) \mathbb{P}_{3} P_{t}^{J} u=g_{\alpha}^{t}\left(\Pi_{0}\right) t^{\alpha-1}\left|\Pi_{0}\right|^{\alpha-1} \mathbb{P}_{3} P_{t}^{J} u
$$

where $g_{\alpha}^{t}$ is as defined in (38). From the bounded holomorphic functional calculus of $\Pi_{0}$ we then obtain

$$
\int_{0}^{\infty}\left\|\mathbb{P}_{3}\left(I-\mathcal{P}_{t}^{J}\right) P_{t}^{J} u\right\|^{2} \frac{d t}{t} \lesssim \int_{0}^{\infty}\left\|\mathbb{P}_{3} t^{\alpha-1}\left|\Pi_{0}\right|^{\alpha-1} P_{t}^{J} u\right\|^{2} \frac{d t}{t}
$$

On recalling that $P_{t}^{J} u=\Gamma_{J} P_{t}^{J}(v, 0,0)$ for some $(v, 0,0) \in D\left(\Gamma_{J}\right)$,

$$
\begin{aligned}
\int_{0}^{\infty}\left\|\mathbb{P}_{3} t^{\alpha-1}\left|\Pi_{0}\right|^{\alpha-1} P_{t}^{J} u\right\|^{2} \frac{d t}{t} & =\int_{0}^{\infty}\left\|\mathbb{P}_{3} t^{\alpha-1}\left|\Pi_{0}\right|^{\alpha-1} \Gamma_{J} P_{t}^{J}(v, 0,0)\right\|^{2} \frac{d t}{t} \\
& =\int_{0}^{\infty}\left\|\mathbb{P}_{3} t^{\alpha-1}\left|\Pi_{0}\right|^{\alpha-1} \Pi_{0} P_{t}^{J}(v, 0,0)\right\|^{2} \frac{d t}{t} .
\end{aligned}
$$

On exploiting the bounded holomorphic functional calculus of the operator $\Pi_{0}$ once more,

$$
\int_{0}^{\infty}\left\|\mathbb{P}_{3} t^{\alpha-1}\left|\Pi_{0}\right|^{\alpha-1} \Pi_{0} P_{t}^{J}(v, 0,0)\right\|^{2} \frac{d t}{t} \lesssim \int_{0}^{\infty}\left\|t^{\alpha-1}\left|\Pi_{0}\right|^{\alpha} P_{t}^{J}(v, 0,0)\right\|^{2} \frac{d t}{t} .
$$

Observe that since $P_{t}^{J}(v, 0,0)$ is non-zero only in the first entry,

$$
\begin{aligned}
\left\|\left|\Pi_{0}\right|^{\alpha} P_{t}^{J}(v, 0,0)\right\| & =\left\|\left(D^{*} D\right)^{\frac{\alpha}{2}}\left(P_{t}^{J}(v, 0,0)\right)_{1}\right\| \\
& \leq b_{\alpha}^{D} \cdot\left\|\left(D_{J}^{*} D_{J}\right)^{\frac{\alpha}{2}}\left(P_{t}^{J}(v, 0,0)\right)_{1}\right\| \\
& =b_{\alpha}^{D} \cdot\left\|\left|\Pi_{J}\right|^{\alpha} P_{t}^{J}(v, 0,0)\right\| .
\end{aligned}
$$

On then applying the bounded holomorphic functional calculus of the operator $\Pi_{J}$,

$$
\left(b_{\alpha}^{D}\right)^{2} \cdot \int_{0}^{\infty}\left\|t^{\alpha-1}\left|\Pi_{J}\right|^{\alpha} P_{t}^{J}(v, 0,0)\right\|^{2} \frac{d t}{t}
$$




$$
\begin{aligned}
& \lesssim\left(b_{\alpha}^{D}\right)^{2} \int_{0}^{\infty}\left\|t^{\alpha-1}\left|\Pi_{J}\right|^{\alpha-1} \Pi_{J} P_{t}^{J}(v, 0,0)\right\|^{2} \frac{d t}{t} \\
& =\left(b_{\alpha}^{D}\right)^{2} \cdot \int_{0}^{\infty}\left\|t^{\alpha-1}\left|\Pi_{J}\right|^{\alpha-1} P_{t}^{J} u\right\|^{2} \frac{d t}{t} \\
& \lesssim(\alpha-1)^{-1}\left(b_{\alpha}^{D}\right)^{2} \cdot\|u\|^{2},
\end{aligned}
$$

where we used the fact that $\Pi_{J}$ is self-adjoint and Corollary 2.1 in the final line.

Combining Propositions 4.1, 4.2 and 4.3 together then gives Theorem 4.1. With this diagonalisation in hand we can now return to our proof of Theorem 3.1. In particular the second component of our square function norm will now be bounded.

Proof of Theorem 3.1.(iii) On splitting the second component of our square function norm from above,

$$
\begin{aligned}
\int_{0}^{\infty}\left\|\Theta_{t}^{J, B} \mathbb{P}_{2} P_{t}^{J} u\right\|^{2} \frac{d t}{t} \lesssim & \int_{0}^{\infty}\left\|\Theta_{t}^{J, B} \mathbb{P}_{2}\left(\mathcal{P}_{t}^{J}-P_{t}^{J}\right) u\right\|^{2} \frac{d t}{t} \\
& +\int_{0}^{\infty}\left\|\Theta_{t}^{J, B} \mathbb{P}_{2} \mathcal{P}_{t}^{J} u\right\|^{2} \frac{d t}{t}
\end{aligned}
$$

The uniform $L^{2}$-boundedness of the operators $\Theta_{t}^{J, B}$ together with Propositions 4.1 and 4.2 give

$$
\int_{0}^{\infty}\left\|\Theta_{t}^{J, B} \mathbb{P}_{2}\left(\mathcal{P}_{t}^{J}-P_{t}^{J}\right) u\right\|^{2} \frac{d t}{t} \lesssim\left(1+\left(b_{\alpha}^{J}\right)^{2}\right)(\alpha-1)^{-1} \cdot\|u\|^{2} .
$$

It remains to bound the second term in the splitting. In order to do so, it will first be shown that $\mathbb{P}_{2} \mathcal{P}_{t}^{J} u \in D\left(\Gamma_{J, B}^{*}\right)$. From the definition of the adjoint, $\mathbb{P}_{2} \mathcal{P}_{t}^{J} u \in D\left(\Gamma_{J, B}^{*}\right)$ if and only if there exists some $u^{\prime} \in \mathcal{H}$ such that

$$
\left\langle\Gamma_{J, B} w, \mathbb{P}_{2} \mathcal{P}_{t}^{J} u\right\rangle=\left\langle w, u^{\prime}\right\rangle
$$

for all $w \in D\left(\Gamma_{J, B}\right)$, where $\Gamma_{J, B}=B_{2}^{*} \Gamma_{J} B_{1}^{*}$. For $w \in D\left(\Gamma_{J, B}\right)$,

$$
\begin{aligned}
\left\langle\Gamma_{J, B} w, \mathbb{P}_{2} \mathcal{P}_{t}^{J} u\right\rangle & =\left\langle B_{2}^{*} \Gamma_{J} B_{1}^{*} w, \mathbb{P}_{2} \mathcal{P}_{t}^{J} u\right\rangle \\
& =\left\langle\Gamma_{J} B_{1}^{*} w, B_{2} \mathbb{P}_{2} \mathcal{P}_{t}^{J} u\right\rangle \\
& =\left\langle M_{J} B_{1}^{*} w, B_{2} \mathbb{P}_{2} \mathcal{P}_{t}^{J} u\right\rangle
\end{aligned}
$$

where in the last line we used the fact that $A_{32}=0$ by $(\mathrm{H} 8 \mathrm{~J} \alpha)$ and therefore $B_{2} \mathbb{P}_{2}=$ $\mathbb{P}_{2} B_{2} \mathbb{P}_{2}$. This proves that $\mathbb{P}_{2} \mathcal{P}_{t}^{J} u \in D\left(\Gamma_{J, B}^{*}\right)$ will follow from $B_{2} \mathbb{P}_{2} \mathcal{P}_{t}^{J} u \in D\left(M_{J}^{*}\right)$ which, in turn, will follow from $\left(\mathcal{P}_{t}^{J} u\right)_{2} \in D\left(J^{*} A_{22}\right)=D\left(J A_{22}\right)$.

Note that $u \in R\left(\Gamma_{J}\right)$ implies that $u=\Gamma_{J}(v, 0,0)$ for some $(v, 0,0) \in D\left(\Gamma_{J}\right)$. Then

$$
\left(\mathcal{P}_{t}^{J} u\right)_{2}=\left(I+t^{2} J^{2}\right)^{-1} J v
$$




$$
=J\left(I+t^{2} J^{2}\right)^{-1} v
$$

$(\mathrm{H} 8 \mathrm{~J} \alpha)$ states that $D\left(J^{2}\right)=D\left(J A_{22} J\right)$ with $\left\|J^{2} \tilde{u}\right\|=\left\|J A_{22} J \tilde{u}\right\|$ for $\tilde{u} \in D\left(J^{2}\right)$. Since $\left(I+t^{2} J^{2}\right)^{-1} v \in D\left(J^{2}\right)$ we must have $\left(I+t^{2} J^{2}\right)^{-1} v \in D\left(J A_{22} J\right)$ and therefore $\left(\mathcal{P}_{t}^{J} u\right)_{2} \in D\left(J A_{22}\right)$. This allows us to conclude that $\mathbb{P}_{2} \mathcal{P}_{t}^{J} u \in D\left(\Gamma_{J, B}^{*}\right)$. Moreover, from (40) we know that $\Gamma_{J, B}^{*} \mathbb{P}_{2} \mathcal{P}_{t}^{J} u=B_{1} M_{J}^{*} B_{2} \mathbb{P}_{2} \mathcal{P}_{t}^{J} u$ and therefore

$$
\begin{aligned}
\left\|\Gamma_{J, B}^{*} \mathbb{P}_{2} \mathcal{P}_{t}^{J} u\right\| & =\left\|B_{1} M_{J}^{*} B_{2} \mathbb{P}_{2} \mathcal{P}_{t}^{J} u\right\| \\
& \lesssim\left\|M_{J}^{*} B_{2} \mathbb{P}_{2} \mathcal{P}_{t}^{J} u\right\| \\
& =\left\|J A_{22} J\left(I+t^{2} J^{2}\right)^{-1} v\right\| \\
& =\left\|J^{2}\left(I+t^{2} J^{2}\right)^{-1} v\right\| \\
& =\left\|J\left(I+t^{2} J^{2}\right)^{-1} u_{2}\right\| .
\end{aligned}
$$

This together with Lemma 3.1 gives

$$
\begin{aligned}
\int_{0}^{\infty}\left\|\Theta_{t}^{J, B} \mathbb{P}_{2} \mathcal{P}_{t}^{J} u\right\|^{2} \frac{d t}{t} & =\int_{0}^{\infty}\left\|P_{t}^{J, B} t \Gamma_{J, B}^{*} \mathbb{P}_{2} \mathcal{P}_{t}^{J} u\right\|^{2} \frac{d t}{t} \\
& \lesssim \int_{0}^{\infty}\left\|t \Gamma_{J, B}^{*} \mathbb{P}_{2} \mathcal{P}_{t}^{J} u\right\|^{2} \frac{d t}{t} \\
& \lesssim \int_{0}^{\infty}\left\|t J\left(I+t^{2} J^{2}\right)^{-1} u_{2}\right\| \frac{d t}{t} .
\end{aligned}
$$

The theorem then follows from the fact that $J$ is self-adjoint and therefore satisfies square function estimates with constant independent of $J$ by Corollary 2.1.

\subsection{The Third Component}

This section is dedicated to bounding the third component of our square function norm and thus proving the fourth and final part of Theorem 3.1. Specifically, it will be proved that when $(\mathrm{H} 8 \mathrm{D} \alpha)$ is satisfied for some $\alpha \in(1,2]$ and either $(\mathrm{H} 8 \mathrm{~J})$ or $(\mathrm{H} 8 \mathrm{~J} \alpha)$ is satisfied the estimate

$$
\int_{0}^{\infty}\left\|\Theta_{t}^{J, B} \mathbb{P}_{3} P_{t}^{J} u\right\|^{2} \frac{d t}{t} \lesssim c_{\alpha}^{J} \cdot\|u\|^{2}
$$

will hold for any $u \in R\left(\Gamma_{J}\right)$. A similar argument to that of [6] will be used, but one will need to keep track of the effect of the projection $\mathbb{P}_{3}$. 


\subsubsection{T(1)-Reduction}

Our first step towards a $T(1)$-reduction is to use the splitting

$$
\begin{aligned}
\int_{0}^{\infty}\left\|\Theta_{t}^{J, B} \mathbb{P}_{3} P_{t}^{J} u\right\|^{2} \frac{d t}{t} \lesssim & \int_{0}^{\infty}\left\|\Theta_{t}^{J, B} \mathbb{P}_{3}\left(P_{t}^{J}-\mathcal{P}_{t}^{J}\right) u\right\|^{2} \frac{d t}{t} \\
& +\int_{0}^{\infty}\left\|\Theta_{t}^{J, B} \mathbb{P}_{3} \mathcal{P}_{t}^{J} u\right\|^{2} \frac{d t}{t}
\end{aligned}
$$

The uniform $L^{2}$-boundedness of the operators $\Theta_{t}^{J, B}$ and Theorem 4.1 can be applied to the first term to obtain

$$
\int_{0}^{\infty}\left\|\Theta_{t}^{J, B} \mathbb{P}_{3}\left(\mathcal{P}_{t}^{J}-P_{t}^{J}\right) u\right\|^{2} \frac{d t}{t} \lesssim c_{\alpha}^{J} \cdot\|u\|^{2} .
$$

On recalling that $\mathbb{P}_{3} \mathcal{P}_{t}^{J}=\mathbb{P}_{3} P_{t}^{0}$, this reduces the task of proving our square function estimate to obtaining the bound

$$
\int_{0}^{\infty}\left\|\Theta_{t}^{J, B} \mathbb{P}_{3} P_{t}^{0} u\right\|^{2} \frac{d t}{t} \lesssim c_{\alpha}^{J} \cdot\|u\|^{2} .
$$

Introduce the notation $\tilde{\Theta}_{t}^{J, B}$ to denote the operators $\tilde{\Theta}_{t}^{J, B}:=\Theta_{t}^{J, B} \mathbb{P}_{3}$. Let $\gamma_{t}^{J, B}$ and $\tilde{\gamma}_{t}^{J, B}$ denote the principal parts of the operators $\Theta_{t}^{J, B}$ and $\tilde{\Theta}_{t}^{J, B}$ respectively. That is, they are the multiplication operators defined through

$$
\gamma_{t}^{J, B}(x) w:=\Theta_{t}^{J, B}(w)(x) \text { and } \tilde{\gamma}_{t}^{J, B}(x)(w):=\left(\Theta_{t}^{J, B} \mathbb{P}_{3}\right)(w)(x),
$$

for $w \in \mathbb{C}^{N}$ and $x \in \mathbb{R}^{n}$. Evidently we must have $\tilde{\gamma}_{t}^{J, B}(x) w=\gamma_{t}^{J, B}(x) \mathbb{P}_{3} w$.

Our square function norm can be reduced to this principal part by applying the splitting

$$
\begin{aligned}
\int_{0}^{\infty}\left\|\tilde{\Theta}_{t}^{J, B} P_{t}^{0} u\right\|^{2} \frac{d t}{t} \lesssim & \int_{0}^{\infty}\left\|\left(\tilde{\Theta}_{t}^{J, B}-\tilde{\gamma}_{t}^{J, B} A_{t}\right) P_{t}^{0} u\right\|^{2} \frac{d t}{t} \\
& +\int_{0}^{\infty}\left\|\tilde{\gamma}_{t}^{J, B} A_{t} P_{t}^{0} u\right\|^{2} \frac{d t}{t}
\end{aligned}
$$

Since the operator $\Theta_{t}^{J, B}$ satisfies the conditions of Proposition 3.6, it follows that

$$
\begin{aligned}
\int_{0}^{\infty}\left\|\left(\tilde{\Theta}_{t}^{J, B}-\tilde{\gamma}_{t}^{J, B} A_{t}\right) P_{t}^{0} u\right\|^{2} \frac{d t}{t} & =\int_{0}^{\infty}\left\|\left(\Theta_{t}^{J, B}-\gamma_{t}^{J, B} A_{t}\right) \mathbb{P}_{3} P_{t}^{0} u\right\|^{2} \frac{d t}{t} \\
& \lesssim \int_{0}^{\infty}\left\|t \nabla \mathbb{P}_{3} P_{t}^{0} u\right\|^{2} \frac{d t}{t} \\
& \lesssim \int_{0}^{\infty}\left\|t \Pi_{0} P_{t}^{0} u\right\|^{2} \frac{d t}{t}
\end{aligned}
$$




$$
\begin{aligned}
& =\int_{0}^{\infty}\left\|Q_{t}^{0} u\right\|^{2} \frac{d t}{t} \\
& =\frac{1}{2}\|u\|^{2},
\end{aligned}
$$

where the estimate $\left\|\nabla \mathbb{P}_{3} P_{t}^{0} u\right\| \lesssim\left\|\Pi_{0} P_{t}^{0} u\right\|$ follows from (H8) for the operator $\Gamma_{0}$. It should be noted that in order to use (H8) we had to use the fact that $u=\Gamma_{J} v$ for some $v \in D\left(\Gamma_{J}\right)$ and therefore

$$
\mathbb{P}_{3} P_{t}^{0} u=P_{t}^{0} \mathbb{P}_{3} \Gamma_{J} v=P_{t}^{0} \mathbb{P}_{3} \Gamma_{0} v=\Gamma_{0} P_{t}^{0} v \in R\left(\Gamma_{0}\right)
$$

Our theorem has thus been reduced to a proof of the following square function estimate

$$
\int_{0}^{\infty}\left\|\tilde{\gamma}_{t}^{J, B} A_{t} P_{t}^{0} u\right\|^{2} \frac{d t}{t} \lesssim c_{\alpha}^{J} \cdot\|u\|^{2} .
$$

On splitting from above using the triangle inequality,

$$
\int_{0}^{\infty}\left\|\tilde{\gamma}_{t}^{J, B} A_{t} P_{t}^{0} u\right\|^{2} \frac{d t}{t} \lesssim \int_{0}^{\infty}\left\|\tilde{\gamma}_{t}^{J, B} A_{t}\left(P_{t}^{0}-I\right) u\right\|^{2} \frac{d t}{t}+\int_{0}^{\infty}\left\|\tilde{\gamma}_{t}^{J, B} A_{t} u\right\|^{2} \frac{d t}{t} .
$$

Proposition 3.5 states that the uniform estimate $\left\|\tilde{\gamma}_{t}^{J, B} A_{t}\right\| \lesssim 1$ is true for all $t>0$. Furthermore, notice that $A_{t}^{2}=A_{t}$ and $\mathbb{P}_{3} A_{t}=A_{t} \mathbb{P}_{3}$ for all $t>0$. These facts combine together to produce

$$
\begin{aligned}
\int_{0}^{\infty}\left\|\tilde{\gamma}_{t}^{J, B} A_{t}\left(P_{t}^{0}-I\right) u\right\|^{2} \frac{d t}{t} & =\int_{0}^{\infty}\left\|\gamma_{t}^{J, B} A_{t} \mathbb{P}_{3} A_{t}\left(P_{t}^{0}-I\right) u\right\|^{2} \frac{d t}{t} \\
& \lesssim \int_{0}^{\infty}\left\|\mathbb{P}_{3} A_{t}\left(P_{t}^{0}-I\right) u\right\|^{2} \frac{d t}{t} .
\end{aligned}
$$

According to the argument from Proposition 5.7 of [6], this final term can be bounded by

$$
\int_{0}^{\infty}\left\|A_{t}\left(P_{t}^{0}-I\right) u\right\|^{2} \frac{d t}{t} \lesssim\|u\|^{2}
$$

since $\left\{\Gamma_{0}, B_{1}, B_{2}\right\}$ by hypothesis satisfies (H1)-(H8). For the second term in (43), apply Carleson's theorem ([22, pg. 59]) to obtain

$$
\int_{0}^{\infty}\left\|\tilde{\gamma}_{t}^{J, B} A_{t} u\right\|^{2} \frac{d t}{t} \lesssim\|\mu\|_{\mathcal{C}} \cdot\|u\|^{2}
$$

where $\mu$ is the measure on $\mathbb{R}^{n+1}$ defined through

$$
d \mu(x, t):=\left|\tilde{\gamma}_{t}^{J, B}(x)\right|^{2} \frac{d x d t}{t}
$$


for $x \in \mathbb{R}^{n}$ and $t>0$ and $\|\mu\|_{\mathcal{C}}$ denotes its Carleson norm. The proof of our theorem has thus been reduced to showing that the measure $\mu$ is a Carleson measure with constant smaller than a multiple of $c_{\alpha}^{J}$.

\subsubsection{Carleson Measure Estimate}

Our goal now is to prove the following Carleson measure estimate,

$$
\sup _{Q \in \Delta} \frac{1}{|Q|} \int_{0}^{l(Q)} \int_{Q}\left|\tilde{\gamma}_{t}^{J, B}(x)\right|^{2} \frac{d x d t}{t} \lesssim c_{\alpha}^{J}<\infty .
$$

Let $\mathcal{L}_{3}$ denote the subspace

$$
\mathcal{L}_{3}:=\left\{v \in \mathcal{L}\left(\mathbb{C}^{N}\right) \backslash\{0\}: v \mathbb{P}_{3}=v\right\}
$$

By construction, we have $\tilde{\gamma}_{t}^{J, B}(x) \in \mathcal{L}_{3}$ for any $t>0$ and $x \in \mathbb{R}^{n}$ since

$$
\begin{aligned}
\tilde{\gamma}_{t}^{J, B}(x) \mathbb{P}_{3} w & =\left(\Theta_{t}^{J, B} \mathbb{P}_{3}\right)\left(\mathbb{P}_{3} w\right)(x) \\
& =\left(\Theta_{t}^{J, B} \mathbb{P}_{3}\right)(w)(x) \\
& =\tilde{\gamma}_{t}^{J, B}(x)(w)
\end{aligned}
$$

Let $\sigma>0$ be a constant to be determined at a later time. Let $\mathcal{V}$ be a finite set consisting of $v \in \mathcal{L}_{3}$ with $|v|=1$ such that $\cup_{v \in \mathcal{V}} K_{v}=\mathcal{L}_{3} \backslash\{0\}$, where

$$
K_{v}:=\left\{v^{\prime} \in \mathcal{L}_{3} \backslash\{0\}:\left|\frac{v^{\prime}}{\left|v^{\prime}\right|}-v\right| \leq \sigma\right\}
$$

Then, in order to prove our Carleson measure estimate (44), it is sufficient to fix $v \in \mathcal{V}$ and prove that

$$
\sup _{Q \in \Delta} \frac{1}{|Q|} \iint_{\substack{(x, t) \in R_{Q} \\ \tilde{\gamma}_{t}^{J, B}(x) \in K_{v}}}\left|\tilde{\gamma}_{t}^{J, B}(x)\right|^{2} \frac{d x d t}{t} \lesssim c_{\alpha}^{J}<\infty
$$

where $R_{Q}:=Q \times[0, l(Q))$. Recall the John-Nirenberg lemma for Carleson measures as applied in [6] and [4].

Lemma 4.1 (The John-Nirenberg Lemma for Carleson Measures) Let $\rho$ be a measure on $\mathbb{R}_{+}^{n+1}$ and $\beta>0$. Suppose that for every $Q \in \Delta$ there exists a collection $\left\{Q_{k}\right\}_{k} \subset \Delta$ of disjoint subcubes of $Q$ such that $E_{Q}:=Q \backslash \cup_{k} Q_{k}$ satisfies $\left|E_{Q}\right|>\beta|Q|$ and such that

$$
\sup _{Q \in \Delta} \frac{\rho\left(E_{Q}^{*}\right)}{|Q|} \leq C
$$


for some $C>0$, where $E_{Q}^{*}:=R_{Q} \backslash \cup_{k} R_{Q_{k}}$. Then

$$
\sup _{Q \in \Delta} \frac{\rho\left(R_{Q}\right)}{|Q|} \leq \frac{C}{\beta}
$$

Proof Fix $Q \in \Delta$ and let $\left\{Q_{k_{1}}\right\}_{k_{1}}$ be a collection of subcubes as in the hypotheses of the lemma. Apply the bound (47) to the decomposition

$$
\rho\left(R_{Q}\right)=\rho\left(E_{Q}^{*}\right)+\sum_{k_{1}} \rho\left(R_{Q_{k_{1}}}\right)
$$

to obtain

$$
\rho\left(R_{Q}\right) \leq C|Q|+\sum_{k_{1}} \rho\left(R_{Q_{k_{1}}}\right)
$$

For each $k_{1}$, let $\left\{Q_{k_{1}, k_{2}}\right\}_{k_{2}}$ be a collection of subcubes of $Q_{k_{1}}$ that satisfy the hypotheses of the lemma. Decompose $\rho\left(R_{Q_{k_{1}}}\right)$ and once again apply (47) to obtain

$$
\begin{aligned}
\rho\left(R_{Q}\right) & \leq C|Q|+\sum_{k_{1}}\left(\rho\left(E_{Q_{k_{1}}}^{*}\right)+\sum_{k_{2}} \rho\left(R_{Q_{k_{1}, k_{2}}}\right)\right) \\
& \leq C|Q|+\sum_{k_{1}} C\left|Q_{k_{1}}\right|+\sum_{k_{1}, k_{2}} \rho\left(R_{Q_{k_{1}, k_{2}}}\right) \\
& \leq C|Q|+C|Q|(1-\beta)+\sum_{k_{1}, k_{2}} \rho\left(R_{Q_{k_{1}, k_{2}}}\right) .
\end{aligned}
$$

Iterating this process and summing the resulting geometric series gives (48).

With this tool at our disposal, the proof of our theorem is reduced to the following proposition.

Proposition 4.4 There exists $\beta>0$ and $\sigma>0$ that will satisfy the following conditions. For every $v \in \mathcal{V}$ and $Q \in \Delta$, there is a collection $\left\{Q_{k}\right\}_{k} \subset \Delta$ of disjoint subcubes of $Q$ such that $E_{Q, v}=Q \backslash \cup_{k} Q_{k}$ satisfies $\left|E_{Q, v}\right|>\beta|Q|$ and such that

$$
\sup _{Q \in \Delta} \frac{1}{|Q|} \iint_{\substack{(x, t) \in E_{Q, v}^{*} \\ \tilde{\gamma}_{t}^{J, B}(x) \in K_{v}}}\left|\tilde{\gamma}_{t}^{J, B}(x)\right|^{2} \frac{d x d t}{t} \lesssim c_{\alpha}^{J}<\infty,
$$

where $E_{Q, v}^{*}:=R_{Q} \backslash \cup_{k} R_{Q_{k}}$. Moreover, $\beta$ and $\sigma$ are entirely independent of the conditions $(H 8 D \alpha),(H 8 J)$ and $(H 8 J \alpha)$.

For now, fix $v \in \mathcal{V}$ and $Q \in \Delta$. Let $w^{\nu}, \hat{w}^{v} \in \mathbb{C}^{N}$ with $\left|\hat{w}^{v}\right|=\left|w^{v}\right|=1$ and $v^{*}\left(\hat{w}^{v}\right)=w^{v}$. To simplify notation, when superfluous, this dependence will be kept 
implicit by defining $w:=w^{v}$ and $\hat{w}:=\hat{w}^{v}$. Notice that since $v$ satisfies $v=v \mathbb{P}_{3}, w$ must satisfy $\mathbb{P}_{3} w=w$.

For $\epsilon>0$ the function $f_{Q, \epsilon}^{w}$ can be defined in an identical manner to [6]. Specifically, let $\eta_{Q}: \mathbb{R}^{n} \rightarrow[0,1]$ be a smooth cutoff function equal to 1 on $2 Q$, with support in $4 Q$ and with $\left\|\nabla \eta_{Q}\right\|_{\infty} \leq \frac{1}{l}$, where $l:=l(Q)$. Then define $w_{Q}:=\eta_{Q} \cdot w$ and

$$
f_{Q, \epsilon}^{w}:=w_{Q}-\epsilon \operatorname{li} \Gamma_{J}\left(I+\epsilon \operatorname{li} \Pi_{J, B}\right)^{-1} w_{Q}=\left(I+\epsilon \operatorname{li} \Gamma_{J, B}^{*}\right)\left(I+\epsilon l i \Pi_{J, B}\right)^{-1} w_{Q} .
$$

Lemma 4.2 There exists a constant $C>0$, independent of $(H 8 D \alpha),(H 8 J)$ and $(H 8 J \alpha)$, that satisfies $\left\|f_{Q, \epsilon}^{w}\right\| \leq C|Q|^{\frac{1}{2}}$ and

$$
\left|f_{Q} \mathbb{P}_{3} f_{Q, \epsilon}^{w}-w\right| \leq C \cdot \epsilon^{\frac{1}{2}},
$$

for any $\epsilon>0$.

Proof The first claim follows from

$$
\begin{aligned}
\left\|f_{Q, \epsilon}^{w}\right\| & \lesssim\left\|w_{Q}\right\|+\left\|\epsilon \operatorname{li} \Gamma_{J}\left(I+\epsilon \operatorname{li} \Pi_{J, B}\right)^{-1} w_{Q}\right\| \\
& \lesssim|Q|^{\frac{1}{2}}+\left\|\epsilon \operatorname{li} \Pi_{J, B}\left(I+\epsilon \operatorname{li} \Pi_{J, B}\right)^{-1} w_{Q}\right\| \\
& \lesssim|Q|^{\frac{1}{2}} .
\end{aligned}
$$

On recalling that $w$ is zero in the first two components,

$$
\begin{aligned}
\left|f_{Q} \mathbb{P}_{3} f_{Q, \epsilon}^{w}-w\right|^{2} & =\left|f_{Q} \mathbb{P}_{3} \epsilon l i \Gamma_{J}\left(I+\epsilon l i \Pi_{J, B}\right)^{-1} w_{Q}\right|^{2} \\
& =\left|f_{Q} \epsilon \operatorname{li} \Gamma_{0}\left(I+\epsilon \operatorname{li} \Pi_{J, B}\right)^{-1} w_{Q}\right|^{2}
\end{aligned}
$$

At this point, apply Lemma 5.6 of [6] to the operator $\Upsilon=\Gamma_{0}$ to obtain

$$
\begin{aligned}
\left|f_{Q} \epsilon \operatorname{li} \Gamma_{0}\left(I+\epsilon \operatorname{li} \Pi_{J, B}\right)^{-1} w_{Q}\right|^{2} & \lesssim \frac{(\epsilon l)^{2}}{l}\left(f_{Q}\left|\left(I+\epsilon \operatorname{li} \Pi_{J, B}\right)^{-1} w_{Q}\right|^{2}\right)^{\frac{1}{2}} \\
& \cdot\left(f_{Q}\left|\Gamma_{0}\left(I+\epsilon l i \Pi_{J, B}\right)^{-1} w_{Q}\right|^{2}\right)^{\frac{1}{2}} \\
& \lesssim \epsilon\left(f_{Q}\left|\epsilon \operatorname{li} \Gamma_{0}\left(I+\epsilon l i \Pi_{J, B}\right)^{-1} w_{Q}\right|^{2}\right)^{\frac{1}{2}} \\
& \leq \epsilon\left(f_{Q}\left|\epsilon \operatorname{li} \Gamma_{J}\left(I+\epsilon l i \Pi_{J, B}\right)^{-1} w_{Q}\right|^{2}\right)^{\frac{1}{2}} \\
& \lesssim \epsilon,
\end{aligned}
$$


where the inequality $\left\|\Gamma_{0} v\right\| \leq\left\|\Gamma_{J} v\right\|$ for $v \in D\left(\Gamma_{J}\right)$ follows trivially from the matrix form of $\Gamma_{0}$ and $\Gamma_{J}$.

Lemma 4.3 There exists a constant $D>0$, independent of $(H 8 D \alpha),(H 8 J)$ and $(H 8 J \alpha)$, such that

$$
\iint_{R_{Q}}\left|\Theta_{t}^{J, B} f_{Q, \epsilon}^{w}(x)\right|^{2} \frac{d x d t}{t} \leq D \frac{|Q|}{\epsilon^{2}} .
$$

Proof First observe that

$$
\begin{aligned}
\Theta_{t}^{J, B} f_{Q, \epsilon}^{w} & =P_{t}^{J, B} t \Gamma_{J, B}^{*}\left(I+\epsilon l i \Gamma_{J, B}^{*}\right)\left(I+\epsilon \operatorname{li} \Pi_{J, B}\right)^{-1} w_{Q} \\
& =\frac{t}{\epsilon l} P_{t}^{J, B} \epsilon l \Gamma_{J, B}^{*}\left(I+\epsilon \operatorname{li} \Pi_{J, B}\right)^{-1} w_{Q} .
\end{aligned}
$$

Therefore

$$
\begin{aligned}
\int_{0}^{l} \int_{Q}\left|\Theta_{t}^{J, B} f_{Q, \epsilon}^{w}(x)\right|^{2} \frac{d x d t}{t} & =\int_{0}^{l}\left(\frac{t}{\epsilon l}\right)^{2} \int_{Q}\left|P_{t}^{J, B} \epsilon l \Gamma_{J, B}^{*}\left(I+\epsilon l i \Pi_{J, B}\right)^{-1} w_{Q}\right|^{2} d x \frac{d t}{t} \\
& \lesssim \int_{0}^{l}\left(\frac{t}{\epsilon l}\right)^{2}\left\|\epsilon l i \Gamma_{J, B}^{*}\left(I+\epsilon l i \Pi_{J, B}\right)^{-1} w_{Q}\right\|^{2} \frac{d t}{t} \\
& \lesssim \frac{|Q|}{(\epsilon l)^{2}} \int_{0}^{l} t d t \\
& \simeq \frac{|Q|}{\epsilon^{2}}
\end{aligned}
$$

From this point forward, with $C$ as in Lemma 4.2, set $\epsilon:=\frac{1}{4 C^{2}}$ and introduce the notation $f_{Q}^{w}:=f_{Q, \epsilon}^{w}$. With this choice of $\epsilon$ it must be true that

$$
\left|f_{Q} \mathbb{P}_{3} f_{Q}^{w}-w\right| \leq \frac{1}{2}
$$

That is,

$$
\begin{aligned}
1-2 \operatorname{Re}\left\langle f_{Q} \mathbb{P}_{3} f_{Q}^{w}, w\right\rangle & =|w|^{2}-2 \operatorname{Re}\left\langle f_{Q} \mathbb{P}_{3} f_{Q}^{w}, w\right\rangle \\
& \leq\left|f_{Q} \mathbb{P}_{3} f_{Q}^{w}-w\right|^{2} \\
& \leq \frac{1}{4}
\end{aligned}
$$

On rearranging we find that

$$
\operatorname{Re}\left\langle f_{Q} \mathbb{P}_{3} f_{Q}^{w}, w\right\rangle \geq \frac{1}{4}
$$


In this context, Lemma 5.11 of [6] will take on the below form.

Lemma 4.4 There exists $\beta, c_{1}, c_{2}>0$ and a collection $\left\{Q_{k}\right\}$ of dyadic cubes of $Q$ such that $\left|E_{Q, v}\right|>\beta|Q|$ and such that

$$
\operatorname{Re}\left\langle w, f_{Q^{\prime}} \mathbb{P}_{3} f_{Q}^{w}\right\rangle \geq c_{1} \text { and } f_{Q^{\prime}}\left|\mathbb{P}_{3} f_{Q}^{w}\right| \leq c_{2}
$$

for all dyadic subcubes $Q^{\prime} \in \Delta$ of $Q$ which satisfy $R_{Q^{\prime}} \cap E_{Q, v}^{*} \neq \emptyset$. Moreover, $\beta, c_{1}$ and $c_{2}$ are independent of $(H 8 D \alpha),(H 8 J),(H 8 J \alpha), Q, \sigma$ and $\nu$.

The proof of this statement follows in an identical manner to the argument in [6]. If we set $\sigma=\frac{c_{1}}{2 c_{2}}$, then the following pointwise estimate can be deduced.

Lemma 4.5 If $(x, t) \in E_{Q, v}^{*}$ and $\tilde{\gamma}_{t}^{J, B}(x) \in K_{v}$ then

$$
\left|\tilde{\gamma}_{t}^{J, B}(x)\left(A_{t} f_{Q}^{w}(x)\right)\right| \geq \frac{1}{2} c_{1}\left|\tilde{\gamma}_{t}^{J, B}(x)\right| .
$$

Proof First observe that

$$
\begin{aligned}
\left|v\left(A_{t} f_{Q}^{w}(x)\right)\right| & \geq \operatorname{Re}\left\langle\hat{w}, v\left(A_{t} f_{Q}^{w}(x)\right)\right\rangle \\
& =\operatorname{Re}\left\langle w, A_{t} f_{Q}^{w}(x)\right\rangle \\
& =\operatorname{Re}\left\langle w, A_{t} \mathbb{P}_{3} f_{Q}^{w}(x)\right\rangle \\
& \geq c_{1} .
\end{aligned}
$$

Then

$$
\begin{aligned}
\left|\frac{\tilde{\gamma}_{t}^{J, B}(x)}{\left|\tilde{\gamma}_{t}^{J, B}(x)\right|}\left(A_{t} f_{Q}^{w}(x)\right)\right| & =\left|\frac{\tilde{\gamma}_{t}^{J, B}(x)}{\left|\tilde{\gamma}_{t}^{J, B}(x)\right|}\left(A_{t} \mathbb{P}_{3} f_{Q}^{w}(x)\right)\right| \\
& \geq\left|v\left(A_{t} f_{Q}^{w}(x)\right)\right|-\left|\frac{\tilde{\gamma}_{t}^{J, B}(x)}{\left|\tilde{\gamma}_{t}^{J, B}(x)\right|}-v\right|\left|A_{t} \mathbb{P}_{3} f_{Q}^{w}(x)\right| \\
& \geq c_{1}-\sigma c_{2} \\
& =\frac{1}{2} c_{1} .
\end{aligned}
$$

Proof of Proposition 4.4 From the pointwise bound of the previous lemma,

$$
\iint_{\substack{(x, t) \in E_{Q, v}^{*} \\ \tilde{\gamma}_{t}^{J, B}(x) \in K_{v}}}\left|\tilde{\gamma}_{t}^{J, B}(x)\right|^{2} \frac{d x d t}{t} \lesssim \iint_{R_{Q}}\left|\tilde{\gamma}_{t}^{J, B}(x) A_{t} f_{Q}^{w}(x)\right|^{2} \frac{d x d t}{t} .
$$


At this stage we can begin to unravel our square function norm,

$$
\begin{gathered}
\iint_{R_{Q}}\left|\tilde{\gamma}_{t}^{J, B}(x) A_{t} f_{Q}^{w}(x)\right|^{2} \frac{d x d t}{t} \lesssim \iint_{R_{Q}}\left|\Theta_{t}^{J, B} f_{Q}^{w}(x)-\tilde{\gamma}_{t}^{J, B}(x) A_{t} f_{Q}^{w}(x)\right|^{2} \frac{d x d t}{t} \\
+\iint_{R_{Q}}\left|\Theta_{t}^{J, B} f_{Q}^{w}(x)\right|^{2} \frac{d x d t}{t}
\end{gathered}
$$

Lemma 4.3 states that the final term in the above estimate will be bounded from above by a multiple of $|Q|$. This reduces the task of proving the proposition to bounding the first term of the above splitting. Recall that $f_{Q}^{w}$ can be expressed in the form

$$
f_{Q}^{w}:=w_{Q}-u_{Q}^{w}
$$

where $u_{Q}^{w} \in R\left(\Gamma_{J}\right)$ is given by

$$
u_{Q}^{w}:=\epsilon \operatorname{li} \Gamma_{J}\left(I+\epsilon \operatorname{li} \Pi_{J, B}\right)^{-1} w_{Q} .
$$

An application of the triangle inequality then leads to

$$
\begin{aligned}
\iint_{R_{Q}}\left|\Theta_{t}^{J, B} f_{Q}^{w}(x)-\tilde{\gamma}_{t}^{J, B}(x) A_{t} f_{Q}^{w}(x)\right|^{2} \frac{d x d t}{t} \\
\lesssim \iint_{R_{Q}}\left|\Theta_{t}^{J, B} w_{Q}(x)-\tilde{\gamma}_{t}^{J, B}(x) A_{t} w_{Q}(x)\right|^{2} \frac{d x d t}{t} \\
\quad+\iint_{R_{Q}}\left|\Theta_{t}^{J, B} u_{Q}^{w}(x)-\tilde{\gamma}_{t}^{J, B}(x) A_{t} u_{Q}^{w}(x)\right|^{2} \frac{d x d t}{t}
\end{aligned}
$$

On noticing that for every $x \in Q$ and $0<t<l(Q)$

$$
\begin{aligned}
\Theta_{t}^{J, B} w_{Q}(x)-\tilde{\gamma}_{t}^{J, B}(x) A_{t} w_{Q}(x) & =\Theta_{t}^{J, B} w_{Q}(x)-\Theta_{t}^{J, B}\left(A_{t} w_{Q}(x)\right)(x) \\
& =\Theta_{t}^{J, B}\left(\left(\eta_{Q}-1\right) w\right)(x),
\end{aligned}
$$

it is clear that the first term in (55) can be handled in an identical manner as in the proof of Proposition 5.9 from [6]. Specifically, since $\left(\operatorname{supp}\left(\eta_{Q}-1\right) w\right) \cap 2 Q=\emptyset$, the off-diagonal estimates of the operator $\Theta_{t}^{J, B}$ lead to

$$
\int_{Q}\left|\Theta_{t}^{J, B}\left(\left(\eta_{Q}-1\right) w\right)(x)\right|^{2} d x \lesssim \frac{t|Q|}{l},
$$

which implies that

$$
\iint_{R_{Q}}\left|\Theta_{t}^{J, B} w_{Q}(x)-\tilde{\gamma}_{t}^{J, B}(x) A_{t} w_{Q}(x)\right|^{2} \frac{d x d t}{t} \lesssim|Q| .
$$


As for the second term in (55),

$$
\begin{aligned}
\iint_{R_{Q}} \mid \Theta_{t}^{J, B} u_{Q}^{w} & -\left.\tilde{\gamma}_{t}^{J, B}(x) A_{t} u_{Q}^{w}(x)\right|^{2} \frac{d x d t}{t} \\
\lesssim & \iint_{R_{Q}}\left|\Theta_{t}^{J, B}\left(I-P_{t}^{J}\right) u_{Q}^{w}(x)\right|^{2} \frac{d x d t}{t} \\
& +\iint_{R_{Q}}\left|\Theta_{t}^{J, B} P_{t}^{J} u_{Q}^{w}(x)-\tilde{\gamma}_{t}^{J, B}(x) A_{t} u_{Q}^{w}(x)\right|^{2} \frac{d x d t}{t}
\end{aligned}
$$

Since $u_{Q}^{w} \in R\left(\Gamma_{J}\right)$, Corollary 3.1 gives

$$
\begin{aligned}
\iint_{R_{Q}}\left|\Theta_{t}^{J, B}\left(I-P_{t}^{J}\right) u_{Q}^{w}\right|^{2} \frac{d x d t}{t} & \lesssim\left\|u_{Q}^{w}\right\|^{2} \\
& \lesssim|Q| .
\end{aligned}
$$

For the remaining term in (56),

$$
\begin{aligned}
\iint_{R_{Q}} \mid \Theta_{t}^{J, B} P_{t}^{J} & u_{Q}^{w}(x)-\left.\tilde{\gamma}_{t}^{J, B}(x) A_{t} u_{Q}^{w}(x)\right|^{2} \frac{d x d t}{t} \\
\lesssim & \iint_{R_{Q}}\left|\Theta_{t}^{J, B}\left(I-\mathbb{P}_{3}\right) P_{t}^{J} u_{Q}^{w}\right|^{2} \frac{d x d t}{t} \\
& +\iint_{R_{Q}}\left|\tilde{\Theta}_{t}^{J, B} P_{t}^{J} u_{Q}^{w}(x)-\tilde{\gamma}_{t}^{J, B}(x) A_{t} u_{Q}^{w}(x)\right|^{2} \frac{d x d t}{t}
\end{aligned}
$$

Since we have already proved the boundedness of the first and second components,

$$
\begin{aligned}
\iint_{R_{Q}}\left|\Theta_{t}^{J, B}\left(I-\mathbb{P}_{3}\right) P_{t}^{J} u_{Q}^{w}\right|^{2} \frac{d x d t}{t} & \lesssim c_{\alpha}^{J} \cdot\left\|u_{Q}^{w}\right\|^{2} \\
& \lesssim c_{\alpha}^{J} \cdot|Q|
\end{aligned}
$$

For the second term in (57),

$$
\begin{aligned}
\iint_{R_{Q}} \mid \tilde{\Theta}_{t}^{J, B} P_{t}^{J} & u_{Q}^{w}(x)-\left.\tilde{\gamma}_{t}^{J, B}(x) A_{t} u_{Q}^{w}(x)\right|^{2} \frac{d x d t}{t} \\
\lesssim & \iint_{R_{Q}}\left|\tilde{\Theta}_{t}^{J, B} P_{t}^{J} u_{Q}^{w}(x)-\tilde{\gamma}_{t}^{J, B}(x) A_{t} P_{t}^{J} u_{Q}^{w}(x)\right|^{2} \frac{d x d t}{t} \\
& +\iint_{R_{Q}}\left|\gamma_{t}^{J, B}(x) \mathbb{P}_{3}\left(A_{t} P_{t}^{J}-A_{t}\right) u_{Q}^{w}(x)\right|^{2} \frac{d x d t}{t}
\end{aligned}
$$

To bound the first term on the right-hand side of the above estimate notice that

$$
\tilde{\Theta}_{t}^{J, B} P_{t}^{J} u_{Q}^{w}(x)-\tilde{\gamma}_{t}^{J, B}(x) A_{t} P_{t}^{J} u_{Q}^{w}(x)=\left(\Theta_{t}^{J, B}-\gamma_{t}^{J, B} A_{t}\right) \mathbb{P}_{3} P_{t}^{J} u_{Q}^{w}(x) .
$$


Theorem 4.1 then allows us to diagonalise our $P_{t}^{J}$ operators in the first term of (58) to get

$$
\begin{aligned}
& \int_{0}^{l(Q)}\left\|\left(\Theta_{t}^{J, B}-\gamma_{t}^{J, B} A_{t}\right) \mathbb{P}_{3} P_{t}^{J} u_{Q}^{w}\right\|_{L^{2}(Q)}^{2} \frac{d t}{t} \lesssim c_{\alpha}^{J}|Q| \\
& +\int_{0}^{\infty}\left\|\left(\Theta_{t}^{J, B}-\gamma_{t}^{J, B} A_{t}\right) \mathbb{P}_{3} \mathcal{P}_{t}^{J} u_{Q}^{w}\right\|^{2} \frac{d t}{t}
\end{aligned}
$$

From Proposition 3.6 we know that

$$
\begin{aligned}
\int_{0}^{\infty}\left\|\left(\Theta_{t}^{J, B}-\gamma_{t}^{J, B} A_{t}\right) \mathbb{P}_{3} \mathcal{P}_{t}^{J} u_{Q}^{w}\right\|^{2} \frac{d t}{t} & \lesssim \int_{0}^{\infty}\left\|t \nabla \mathbb{P}_{3} \mathcal{P}_{t}^{J} u_{Q}^{w}\right\|^{2} \frac{d t}{t} \\
& =\int_{0}^{\infty}\left\|t \nabla \mathbb{P}_{3} P_{t}^{0} u_{Q}^{w}\right\|^{2} \frac{d t}{t} \\
& \lesssim \int_{0}^{\infty}\left\|t \Pi_{0} P_{t}^{0} u_{Q}^{w}\right\|^{2} \frac{d t}{t} \\
& =\int_{0}^{\infty}\left\|Q_{t}^{0} u_{Q}^{w}\right\|^{2} \frac{d t}{t} \\
& \lesssim|Q|,
\end{aligned}
$$

where in the third line we applied (H8) for the operators $\left\{\Gamma_{0}, B_{1}, B_{2}\right\}$. It should be noted that in order to use (H8), we had to use the fact that

$$
\begin{aligned}
\mathbb{P}_{3} P_{t}^{0} u_{Q}^{w} & =P_{t}^{0} \mathbb{P}_{3} u_{Q}^{w}=P_{t}^{0} \mathbb{P}_{3} \epsilon l i \Gamma_{J}\left(I+\epsilon l i \Pi_{J, B}\right)^{-1} w_{Q} \\
& =P_{t}^{0} \mathbb{P}_{3} \epsilon l i \Gamma_{0}\left(I+\epsilon l i \Pi_{J, B}\right)^{-1} w_{Q}=\epsilon l i \Gamma_{0} P_{t}^{0}\left(I+\epsilon l i \Pi_{J, B}\right)^{-1} w_{Q} \in R\left(\Gamma_{0}\right)
\end{aligned}
$$

It remains to bound the second term in (58),

$$
\begin{aligned}
& \iint_{R_{Q}}\left|\gamma_{t}^{J, B}(x) \mathbb{P}_{3} A_{t}\left(P_{t}^{J}-I\right) u_{Q}^{w}(x)\right|^{2} \frac{d x d t}{t} \\
& =\iint_{R_{Q}}\left|\gamma_{t}^{J, B} A_{t} \mathbb{P}_{3} A_{t}\left(P_{t}^{J}-I\right) u_{Q}^{w}(x)\right|^{2} \frac{d x d t}{t}
\end{aligned}
$$

On noting the uniform $L^{2}$-boundedness of the $\gamma_{t}^{J, B} A_{t}$ operators and applying the triangle inequality,

$$
\begin{aligned}
& \iint_{R_{Q}}\left|\gamma_{t}^{J, B} A_{t} \mathbb{P}_{3} A_{t}\left(P_{t}^{J}-I\right) u_{Q}^{w}(x)\right|^{2} \frac{d x d t}{t} \\
& \lesssim \int_{0}^{\infty} \int_{\mathbb{R}^{n}}\left|\mathbb{P}_{3} A_{t}\left(P_{t}^{J}-I\right) u_{Q}^{w}(x)\right|^{2} \frac{d x d t}{t} \\
& \lesssim \int_{0}^{\infty} \int_{\mathbb{R}^{n}}\left|\mathbb{P}_{3} A_{t}\left(P_{t}^{J}-\mathcal{P}_{t}^{J}\right) u_{Q}^{w}(x)\right|^{2} \frac{d x d t}{t}
\end{aligned}
$$




$$
+\int_{0}^{\infty} \int_{\mathbb{R}^{n}}\left|\mathbb{P}_{3} A_{t}\left(\mathcal{P}_{t}^{J}-I\right) u_{Q}^{w}(x)\right|^{2} \frac{d x d t}{t}
$$

Applying Theorem 4.1 and recalling that $\mathbb{P}_{3} \mathcal{P}_{t}^{J}=\mathbb{P}_{3} P_{t}^{0}$,

$$
\begin{aligned}
& \iint_{R_{Q}}\left|\gamma_{t}^{J, B} A_{t} \mathbb{P}_{3} A_{t}\left(P_{t}^{J}-I\right) u_{Q}^{w}(x)\right|^{2} \frac{d x d t}{t} \lesssim c_{\alpha}^{J} \cdot\|u\|^{2} \\
& +\int_{0}^{\infty} \int_{\mathbb{R}^{n}}\left|\mathbb{P}_{3} A_{t}\left(P_{t}^{0}-I\right) u_{Q}^{w}(x)\right|^{2} \frac{d x d t}{t}
\end{aligned}
$$

From the proof of Proposition 5.7 of [6] we know that

$$
\int_{0}^{\infty} \int_{\mathbb{R}^{n}}\left|A_{t}\left(P_{t}^{0}-I\right) u_{Q}^{w}(x)\right|^{2} \frac{d x d t}{t} \lesssim|Q|,
$$

allowing us to finally conclude our proof.

\section{Applications}

Our non-homogeneous framework will now be applied to three different contexts. We begin with the case that serves as the primary motivation for this article, the scalar Kato square root problem with zero-order potential.

\subsection{Scalar Kato with Zero-Order Potential}

Theorem 1.2, the promised result of the introductory section, will now be proved. Fix $V \in \mathcal{W}_{\alpha}$ for some $\alpha \in(1,2]$. Brand the definition of the operators $\Gamma_{J}, B_{1}$ and $B_{2}$ to be as follows. Define our Hilbert space to be

$$
\mathcal{H}:=L^{2}\left(\mathbb{R}^{n}\right) \oplus L^{2}\left(\mathbb{R}^{n}\right) \oplus L^{2}\left(\mathbb{R}^{n} ; \mathbb{C}^{n}\right)
$$

for some $n \in \mathbb{N}^{*}$. Set $J=|V|^{\frac{1}{2}}$ and $D=\nabla$. Our operator $\Gamma_{J}$ is then given by

$$
\Gamma_{J}=\Gamma_{|V|^{\frac{1}{2}}}=\left(\begin{array}{cc}
0 & 0 \\
\nabla_{|V|^{\frac{1}{2}}} & 0
\end{array}\right)=\left(\begin{array}{ccc}
0 & 0 & 0 \\
|V|^{\frac{1}{2}} & 0 & 0 \\
\nabla & 0 & 0
\end{array}\right)
$$

defined on the dense domain $H^{1, V}\left(\mathbb{R}^{n}\right) \oplus L^{2}\left(\mathbb{R}^{n}\right) \oplus L^{2}\left(\mathbb{R}^{n} ; \mathbb{C}^{n}\right)$, where $H^{1, V}\left(\mathbb{R}^{n}\right)$ is as defined in the introductory section. The density of $H^{1, V}\left(\mathbb{R}^{n}\right)$ in $L^{2}\left(\mathbb{R}^{n}\right)$ follows from $V \in L_{\text {loc }}^{1}\left(\mathbb{R}^{n}\right)$.

Let $A \in L^{\infty}\left(\mathbb{R}^{n} ; \mathcal{L}\left(\mathbb{C}^{n}\right)\right)$ be a matrix-valued multiplication operator and suppose that the Gårding inequalities (1) and (6) are satisfied with constants $\kappa^{A}>0$ and 
$\kappa_{V}^{A}>0$ respectively. Define our perturbations $B_{1}$ and $B_{2}$ through

$$
B_{1}=I \quad \text { and } \quad B_{2}:=\left(\begin{array}{cc}
I & 0 \\
0 & \hat{A}
\end{array}\right):=\left(\begin{array}{ccc}
I & 0 & 0 \\
0 & e^{i \cdot \arg V} & 0 \\
0 & 0 & A
\end{array}\right) .
$$

Our perturbed Dirac-type operator then becomes

$$
\Pi_{B,|V|^{\frac{1}{2}}}:=\Gamma_{|V|^{\frac{1}{2}}}+\Gamma_{|V|^{\frac{1}{2}}}^{*}\left(\begin{array}{ll}
I & 0 \\
0 & \hat{A}
\end{array}\right)=\left(\begin{array}{cc}
0 & \nabla^{*} \hat{\frac{1}{A}} \hat{A} \\
\nabla_{|V|^{\frac{1}{2}}} & 0
\end{array}\right) .
$$

The square of our perturbed Dirac-type operator is then given by

$$
\Pi_{B,|V|^{\frac{1}{2}}}^{2}=\left(\begin{array}{ccc}
\nabla_{|V|^{\frac{1}{2}}} \hat{A} \nabla_{|V|^{\frac{1}{2}}} & 0 \\
& 0 & \nabla_{|V|^{\frac{1}{2}}} \nabla_{|V|^{\frac{1}{2}}}^{*} \hat{A}
\end{array}\right)=\left(\begin{array}{cc}
-\operatorname{div}(A \nabla)+V & 0 \\
0 & \nabla_{|V|^{\frac{1}{2}}} \nabla_{|V|^{\frac{1}{2}}}^{*} \hat{A}
\end{array}\right) .
$$

It is clear from the form of our operator $\Gamma_{0}$ and the fact that $A$ satisfies (1) that the operators $\left\{\Gamma_{0}, B_{1}, B_{2}\right\}$ satisfy (H1)-(H8). Similarly, since $A$ and $V$ satisfy (6), it follows that $\left\{\Gamma_{J}, B_{1}, B_{2}\right\}$ satisfy the properties (H1)-(H6).

Lemma 5.1 For $V \in \mathcal{W}_{\alpha}$, the conditions $(H 8 D \alpha)$ and $(H 8 J \alpha)$ are both satisfied and $c_{\alpha}^{J} \lesssim\left(1+[V]_{\alpha}^{2}\right)(\alpha-1)^{-1}$.

Proof The condition $V \in \mathcal{W}_{\alpha}$ tells us that

$$
\left\||V|^{\frac{\alpha}{2}} u\right\|_{2}+\left\|(-\Delta)^{\frac{\alpha}{2}} u\right\|_{2} \leq[V]_{\alpha}\left\|(|V|-\Delta)^{\frac{\alpha}{2}} u\right\|_{2}
$$

for all $u \in D(|V|-\Delta)$. Let's first prove that $(\mathrm{H} 8 \mathrm{D} \alpha)$ is satisfied. In order to prove this condition, it is sufficient to show that $D\left((|V|-\Delta)^{\frac{\alpha}{2}}\right) \subset D\left((-\Delta)^{\frac{\alpha}{2}}\right)$ and

$$
\left\|(-\Delta)^{\frac{\alpha}{2}} u\right\|_{2} \leq[V]_{\alpha}\left\|(|V|-\Delta)^{\frac{\alpha}{2}} u\right\|_{2}
$$

for all $u \in D\left((|V|-\Delta)^{\frac{\alpha}{2}}\right)$. Fix $u \in D\left((|V|-\Delta)^{\frac{\alpha}{2}}\right)$. Since $D(|V|-\Delta)$ is a core for $(|V|-\Delta)^{\frac{\alpha}{2}}$, there must exist some $\left\{u_{n}\right\}_{n=1}^{\infty} \subset D(|V|-\Delta)$ with

$$
\left\|u_{n}-u\right\|_{2}+\left\|(|V|-\Delta)^{\frac{\alpha}{2}}\left(u_{n}-u\right)\right\|_{2} \stackrel{n \rightarrow \infty}{\longrightarrow} 0 .
$$

We then have for $n, m \in \mathbb{N}$,

$$
\left\|u_{n}-u_{m}\right\|_{2}+\left\|(-\Delta)^{\frac{\alpha}{2}}\left(u_{n}-u_{m}\right)\right\|_{2} \leq\left\|u_{n}-u_{m}\right\|_{2}+[V]_{\alpha}\left\|(|V|-\Delta)^{\frac{\alpha}{2}}\left(u_{n}-u_{m}\right)\right\|_{2} .
$$


This proves that $\left\{u_{n}\right\}_{n=1}^{\infty}$ is Cauchy in the graph norm of $(-\Delta)^{\frac{\alpha}{2}}$. The sequence $\left\{u_{n}\right\}_{n=1}^{\infty}$ must therefore converge to some $\tilde{u} \in D\left((-\Delta)^{\frac{\alpha}{2}}\right)$ in the graph norm of $(-\Delta)^{\frac{\alpha}{2}}$,

$$
\left\|u_{n}-\tilde{u}\right\|_{2}+\left\|(-\Delta)^{\frac{\alpha}{2}}\left(u_{n}-\tilde{u}\right)\right\|_{2} \stackrel{n \rightarrow \infty}{\longrightarrow} 0 .
$$

This combined with (61) shows that $u=\tilde{u}$ and therefore $u \in D\left((-\Delta)^{\frac{\alpha}{2}}\right)$. Moreover, we have that

$$
\begin{aligned}
\left\|(-\Delta)^{\frac{\alpha}{2}} u\right\|_{2} & =\lim _{n \rightarrow \infty}\left\|(-\Delta)^{\frac{\alpha}{2}} u_{n}\right\|_{2} \\
& \leq[V]_{\alpha} \lim _{n \rightarrow \infty}\left\|(|V|-\Delta)^{\frac{\alpha}{2}} u_{n}\right\|_{2} \\
& =[V]_{\alpha}\left\|(|V|-\Delta)^{\frac{\alpha}{2}} u\right\|_{2},
\end{aligned}
$$

completing the proof of $(\mathrm{H} 8 \mathrm{D} \alpha)$ with $b_{\alpha}^{D} \lesssim[V]_{\alpha}$. An identical proof can be used to obtain the condition $(\mathrm{H} 8 \mathrm{~J} \alpha)$ with constant $b_{\alpha}^{J} \lesssim[V]_{\alpha}$.

Combining the above Lemma with Corollary 3.2 completes the proof of Theorem 1.2 .

\subsection{The Class $\mathcal{W}$}

Define the potential class

$$
\mathcal{W}:=\bigcup_{\alpha \in(1,2]} \mathcal{W}_{\alpha}
$$

It has so far been proved that the Kato estimate holds for any potential in the class $\mathcal{W}$. At this stage, however, the unperturbed condition $V \in \mathcal{W}$ remains in quite an abstract form. It will therefore be instructive to unpack this condition and compare $\mathcal{W}$ with other commonly used classes of potentials. It is first interesting to note that $\mathcal{W}_{1}$ is the collection of all potentials with no additional restrictions.

Proposition 5.1 For any locally integrable $V: \mathbb{R}^{n} \rightarrow \mathbb{C}$ we have $[V]_{1} \leq 2$. That is, $\mathcal{W}_{1}=L_{\text {loc }}^{1}\left(\mathbb{R}^{n}\right)$.

Proof We have

$$
\begin{aligned}
\left\|(-\Delta)^{\frac{1}{2}} u\right\|_{2}^{2} & =\left\langle(-\Delta)^{\frac{1}{2}} u,(-\Delta)^{\frac{1}{2}} u\right\rangle_{2}=\langle(-\Delta) u, u\rangle_{2} \\
& \leq\langle-\Delta u, u\rangle_{2}+\langle|V| u, u\rangle_{2} \\
& =\left\langle(|V|-\Delta)^{\frac{1}{2}} u,(|V|-\Delta)^{\frac{1}{2}} u\right\rangle_{2}=\left\|(|V|-\Delta)^{\frac{1}{2}} u\right\|_{2}^{2}
\end{aligned}
$$

for all $u \in D(|V|-\Delta)$. The second estimate follows in a similar manner from the non-negativity of $(-\Delta)$. 
Using this as an endpoint, it can then be proved using an interpolation style argument that the potential classes $\left\{\mathcal{W}_{\alpha}\right\}_{\alpha \in[1,2]}$ form a decreasing collection.

Proposition 5.2 Suppose that the potential $V: \mathbb{R}^{n} \rightarrow \mathbb{C}$ is in $\mathcal{W}_{\alpha}$ for some $\alpha \in(1,2]$. Then $V \in \mathcal{W}_{\beta}$ for any $\beta \in[1, \alpha]$ with

$$
[V]_{\beta} \leq 2[V]_{\alpha}^{\frac{\beta-1}{\alpha-1}}
$$

Therefore for $\beta \leq \alpha$

$$
\mathcal{W}_{\beta} \supset \mathcal{W}_{\alpha}
$$

Proof Assume that $V \in \mathcal{W}_{\alpha}$ for some $\alpha \in(1,2]$. It will be shown through the Hadamard three-lines theorem that $V \in \mathcal{W}_{\beta}$ for $\beta \in[1, \alpha]$ with the constant given in (62).

Let $S t:=\{z \in \mathbb{C}: 0<\operatorname{Re} z<1\}$ and set $\theta:=\frac{\beta-1}{\alpha-1}$. Fix $u \in D(|V|-\Delta)$ and define $f: \overline{S t} \rightarrow \mathbb{R}$ to be the function given by

$$
f(z):=\left\||V|^{\frac{1}{2}+\left(\frac{\alpha-1}{2}\right) z}(|V|-\Delta)^{\left(\frac{\alpha-1}{2}\right)(\theta-z)} u\right\|_{2} .
$$

$f$ is holomorphic on $S t$ and continuous on the closed strip $\overline{S t}$. In order to apply the three-lines theorem, it must first be proved that $f$ is bounded on $\overline{S t}$. For $z=s+i t$ where $0 \leq s \leq 1$ and $t \in \mathbb{R}$ we have

$$
\begin{aligned}
f(z) & =\left\||V|^{\frac{1}{2}+\left(\frac{\alpha-1}{2}\right)(s+i t)}(|V|-\Delta)^{\frac{\beta-1}{2}-\left(\frac{\alpha-1}{2}\right)(s+i t)} u\right\|_{2} \\
& =\left\||V|^{\frac{1}{2}+\left(\frac{\alpha-1}{2}\right) s}(|V|-\Delta)^{-\frac{1}{2}-\left(\frac{\alpha-1}{2}\right) s}(|V|-\Delta)^{-\left(\frac{\alpha-1}{2}\right) i t}(|V|-\Delta)^{\frac{\beta}{2}} u\right\|_{2} .
\end{aligned}
$$

The function

$$
s \mapsto\left\||V|^{\frac{1}{2}+\left(\frac{\alpha-1}{2}\right) s}(|V|-\Delta)^{-\frac{1}{2}-\left(\frac{\alpha-1}{2}\right) s} v\right\|_{2}
$$

is continuous on $[0,1]$ for $v \in L^{2}\left(\mathbb{R}^{n}\right)$. This, together with (63) then gives

$$
\begin{aligned}
f(z) & \lesssim\left\|(|V|-\Delta)^{-\left(\frac{\alpha-1}{2}\right) i t}(|V|-\Delta)^{\frac{\beta}{2}} u\right\|_{2} \\
& \leq\left\|(|V|-\Delta)^{\frac{\beta}{2}} u\right\|_{2},
\end{aligned}
$$

where we used the fact that $(|V|-\Delta)^{i a}$ is a contraction operator on $L^{2}\left(\mathbb{R}^{n}\right)$ for $a \in \mathbb{R}$. This demonstrates that $f$ is bounded on $\overline{S t}$. 
For $t \in \mathbb{R}$, Proposition 5.1 implies that

$$
\begin{aligned}
f(i t) & =\left\||V|^{\frac{1}{2}+\left(\frac{\alpha-1}{2}\right) i t}(|V|-\Delta)^{\left(\frac{\alpha-1}{2}\right)(\theta-i t)} u\right\|_{2} \\
& =\left\||V|^{\frac{1}{2}}(|V|-\Delta)^{\left(\frac{\alpha-1}{2}\right)(\theta-i t)} u\right\|_{2} \\
& \leq\left\|(|V|-\Delta)^{\frac{1}{2}}(|V|-\Delta)^{\left(\frac{\alpha-1}{2}\right)(\theta-i t)} u\right\|_{2} \\
& \leq\left\|(|V|-\Delta)^{\frac{\beta}{2}} u\right\|_{2} .
\end{aligned}
$$

We also have

$$
\begin{aligned}
f(1+i t) & =\left\||V|^{\frac{\alpha}{2}+\left(\frac{\alpha-1}{2}\right) i t}(|V|-\Delta)^{\left(\frac{\alpha-1}{2}\right)(\theta-1-i t)} u\right\|_{2} \\
& =\left\||V|^{\frac{\alpha}{2}}(|V|-\Delta)^{\left(\frac{\alpha-1}{2}\right)(\theta-1-i t)} u\right\|_{2} \\
& \leq[V]_{\alpha}\left\|(|V|-\Delta)^{\frac{\alpha}{2}}(|V|-\Delta)^{\left(\frac{\alpha-1}{2}\right)(\theta-1-i t)} u\right\|_{2} \\
& \leq[V]_{\alpha}\left\|(|V|-\Delta)^{\frac{\alpha}{2}+\left(\frac{\alpha-1}{2}\right)(\theta-1)} u\right\|_{2} \\
& =[V]_{\alpha}\left\|(|V|-\Delta)^{\frac{\beta}{2}} u\right\|_{2} .
\end{aligned}
$$

The Hadamard three-lines theorem then gives the bound

$$
f(\theta)=\left\||V|^{\frac{\beta}{2}} u\right\|_{2} \leq[V]_{\alpha}^{\left(\frac{\beta-1}{\alpha-1}\right)}\left\|(|V|-\Delta)^{\frac{\beta}{2}} u\right\|_{2} .
$$

A similar argument can be applied to obtain the bound

$$
\left\|(-\Delta)^{\frac{\beta}{2}} u\right\|_{2} \leq[V]_{\alpha}^{\left(\frac{\beta-1}{\alpha-1}\right)}\left\|(|V|-\Delta)^{\frac{\beta}{2}} u\right\|_{2}
$$

for all $u \in D(|V|-\Delta)$, one must simply remember that the imaginary powers of the positive self-adjoint operator $(-\Delta)$ are contraction operators on $L^{2}\left(\mathbb{R}^{n}\right)$. Combining (64) and (65) then gives $[V]_{\beta} \leq 2[V]_{\alpha}^{\frac{\beta-1}{\alpha-1}}$.

Recall that a non-negative potential $V \in L_{\text {loc }}^{q}\left(\mathbb{R}^{n}\right)$ for index $1<q<\infty$ is said to belong to the reverse Hölder class $R H_{q}$ if there exists $C>0$ such that

$$
\left(\frac{1}{|B|} \int_{B} V^{q} d x\right)^{\frac{1}{q}} \leq C\left(\frac{1}{|B|} \int_{B} V d x\right)
$$


for every ball $B \subset \mathbb{R}^{n}$. For $V \in R H_{q}$, the condition $[V]_{\alpha}<\infty$ for $\alpha=2$ was proved in [21] for $q \geq \frac{n}{2}$ and $n \geq 3$. This was later improved to $q \geq 2$ and arbitrary dimension in [2]. Note that in [2, Thm. 1.1] the estimate $\|\Delta u\|_{2}+\||V| u\|_{2} \lesssim\|(|V|-\Delta) u\|_{2}$ was proved for $u \in C_{0}^{\infty}\left(\mathbb{R}^{n}\right)$. However, this estimate can then be extended to all of $D(|V|-\Delta)$ since $|V| \in L_{l o c}^{2}\left(\mathbb{R}^{n}\right)$ implies that $C_{0}^{\infty}\left(\mathbb{R}^{n}\right)$ is a core for $(|V|-\Delta)$ (c.f. [20]).

Theorem 5.1 ([21], [2]) Let $V \in L_{\text {loc }}^{1}\left(\mathbb{R}^{n}\right)$ and suppose that $|V| \in R H_{q}$ for some $q \geq 2$. Then $V \in \mathcal{W}_{2} \subset \mathcal{W}$.

Notice that since the absolute value of any polynomial is contained in $R H_{q}$ for any $q \in(1, \infty)$ we automatically obtain the following corollary.

Corollary 5.1 For any polynomial $P$, we have $P \in \mathcal{W}_{2}$. As a result, the Kato estimate holds for any polynomial with range contained in $S_{\mu+}$ for some $\mu \in\left[0, \frac{\pi}{2}\right)$.

The ensuing proposition demonstrates that the inclusion of the reverse Hölder potentials in $\mathcal{W}_{2}$ is strict, at least in dimension $n>4$.

Proposition 5.3 For $n>4$,

$$
L^{\frac{n}{2}}\left(\mathbb{R}^{n}\right) \subset \mathcal{W}_{2}
$$

Proof Fix $V \in L^{\frac{n}{2}}\left(\mathbb{R}^{n}\right)$. For $\varepsilon>0$, the resolvent $(|V|+\varepsilon-\Delta)^{-1}$ is well-defined as a bounded operator on $L^{2}\left(\mathbb{R}^{n}\right)$. Hölder's inequality followed by the fact that $(|V|+$ $\varepsilon-\Delta)^{-1} u \leq(-\Delta+\varepsilon)^{-1} u$ for any $u \in L^{2}$ and then finally the uniform boundedness of $(-\Delta+\varepsilon)^{-1}$ from $L^{\frac{2 n}{n-4}}$ to $L^{2}$ (c.f. [13, Sect. 6.1.2]) produces

$$
\begin{aligned}
\|(|V|+\varepsilon) & (|V|+\varepsilon-\Delta)^{-1} u \|_{L^{2}(B(0, N))} \\
& \leq\||V|+\varepsilon\|_{L^{\frac{n}{2}}(B(0, N))} \cdot\left\|(|V|+\varepsilon-\Delta)^{-1} u\right\|_{L^{\frac{2 n}{n-4}}(B(0, N))} \\
& \leq\||V|+\varepsilon\|_{L^{\frac{n}{2}}(B(0, N))} \cdot\left\|(-\Delta+\varepsilon)^{-1} u\right\|_{L^{\frac{2 n}{n-4}}(B(0, N))} \\
& \lesssim\||V|+\varepsilon\|_{L^{\frac{n}{2}}(B(0, N))}\|u\|_{2}
\end{aligned}
$$

for any $N>0$, where $B(0, N) \subset \mathbb{R}^{n}$ is the open ball of radius $N$ centered at the origin. Therefore,

$$
\begin{aligned}
\|(|V|+\varepsilon) u\|_{L^{2}(B(0, N))} \lesssim\||V|+\varepsilon\|_{L^{\frac{n}{2}(B(0, N))}} \cdot\|(|V|+\varepsilon-\Delta) u\|_{2} \\
\leq\||V|+\varepsilon\|_{L^{\frac{n}{2}}(B(0, N))} \cdot\left(\|(|V|-\Delta) u\|_{2}+\varepsilon\|u\|_{2}\right)
\end{aligned}
$$

for all $u \in D(|V|+\varepsilon-\Delta)=D(|V|-\Delta)$. Letting $\varepsilon \rightarrow 0$ followed by $N \rightarrow \infty$ gives the estimate

$$
\||V| u\|_{2} \lesssim\|V\|_{\frac{n}{2}} \cdot\|(|V|-\Delta) u\|_{2}
$$


for all $u \in D(|V|-\Delta)$. The triangle inequality then yields the bound $\|(-\Delta) u\|_{2} \lesssim$ $\|(|V|-\Delta) u\|_{2}$ and thus $V \in \mathcal{W}_{2}$.

The above statements demonstrate clearly that the class of potentials $\mathcal{W}_{2}$ is quite large. In light of Proposition 5.2, however, it is also evident that $\mathcal{W}_{2}$ is the smallest class out of the collection $\left\{\mathcal{W}_{\alpha}\right\}_{\alpha \in(1,2]}$. One can then neatly surmise that $\mathcal{W}$, the class of all potentials for which the Kato estimate holds, is certain to be quite large itself.

\subsection{Systems with Zero-Order Potential}

Fix $m \in \mathbb{N}^{*}$ and $A \in L^{\infty}\left(\mathbb{R}^{n} ; \mathcal{L}\left(\mathbb{C}^{n} \otimes \mathbb{C}^{m}\right)\right)$. Let $V: \mathbb{R}^{n} \rightarrow \mathcal{L}\left(\mathbb{C}^{m}\right)$ be a measurable matrix-valued function contained in $L_{\text {loc }}^{1}\left(\mathbb{R}^{n} ; \mathcal{L}\left(\mathbb{C}^{m}\right)\right)$ with $V(x)$ normal for almost every $x \in \mathbb{R}^{n}$. $V$ can be viewed as a densely defined closed multiplication operator on $L^{2}\left(\mathbb{R}^{n} ; \mathbb{C}^{m}\right)$ with domain

$$
D(V)=\left\{u \in L^{2}\left(\mathbb{R}^{n} ; \mathbb{C}^{m}\right): V \cdot u \in L^{2}\left(\mathbb{R}^{n} ; \mathbb{C}^{m}\right)\right\}
$$

Similar to the scalar case, one can define forms $\mathfrak{l}^{A}$ and $\mathfrak{l}_{V}^{A}$ respectively through

$$
\mathfrak{l}^{A}[u, v]:=\int_{\mathbb{R}^{n}}\langle A(x) \nabla u(x), \nabla v(x)\rangle_{\mathbb{C}^{n} \otimes \mathbb{C}^{m}} d x
$$

for $u, v \in H^{1}\left(\mathbb{R}^{n} ; \mathbb{C}^{m}\right)$ and

$$
\mathfrak{l}_{V}^{A}\left[u^{\prime}, v^{\prime}\right]:=\mathfrak{l}^{A}\left[u^{\prime}, v^{\prime}\right]+\int_{\mathbb{R}^{n}}\left\langle V(x) u^{\prime}(x), v^{\prime}(x)\right\rangle_{\mathbb{C}^{m}} d x
$$

for $u^{\prime}$ and $v^{\prime}$ contained in

$$
H^{1, V}\left(\mathbb{R}^{n} ; \mathbb{C}^{m}\right):=H^{1}\left(\mathbb{R}^{n} ; \mathbb{C}^{m}\right) \cap D\left(|V|^{\frac{1}{2}}\right),
$$

where $|V(x)|:=\sqrt{V(x)^{*} V(x)}$ for $x \in \mathbb{R}^{n}$. The density of $H^{1, V}\left(\mathbb{R}^{n} ; \mathbb{C}^{m}\right)$ in $L^{2}\left(\mathbb{R}^{n} ; \mathbb{C}^{m}\right)$ follows from the fact that $C_{0}^{\infty}\left(\mathbb{R}^{n} ; \mathbb{C}^{m}\right) \subset H^{1, V}\left(\mathbb{R}^{n} ; \mathbb{C}^{m}\right)$. Assume that the forms $\mathfrak{l}^{A}$ and $\mathfrak{l}_{V}^{A}$ satisfy the system equivalents of the Gårding inequalities (1) and (6) with constants $\kappa^{A}>0$ and $\kappa_{V}^{A}>0$ respectively. Then $\mathfrak{l}^{A}$ and $\mathfrak{l}_{V}^{A}$ will both have a unique associated maximal accretive operator, $L$ and $L+V$.

In the article [3], the Kato square root property $\|\sqrt{L} u\|_{2} \simeq\|\nabla u\|_{2}$ was proved for elliptic systems without potential. Using the non-homogeneous machinery that we have developed, the corresponding property can be proved for the operator with potential. Define $[V]_{\alpha}$ and $\mathcal{W}_{\alpha}\left(\mathbb{R}^{n} ; \mathcal{L}\left(\mathbb{C}^{m}\right)\right)$ for $\alpha \in[1,2]$ to be the system analogues of the corresponding scalar objects. In the below theorem, our non-homogeneous framework will be applied to determine the domain of $\sqrt{L+V}$ for normal potentials.

Theorem 5.2 Suppose that $V \in \mathcal{W}_{\alpha}\left(\mathbb{R}^{n} ; \mathcal{L}\left(\mathbb{C}^{m}\right)\right)$ for some $\alpha \in(1,2]$ and that $V(x)$ is a normal matrix for almost every $x \in \mathbb{R}^{n}$. Suppose that the system equivalents 
of the Gårding inequalities (1) and (6) are both satisfied with constants $\kappa^{A}$ and $\kappa_{V}^{A}$ respectively. Then there must exist some $C_{V}>0$ such that

$$
C_{V}^{-1}\left(\left\||V|^{\frac{1}{2}} u\right\|_{2}+\|\nabla u\|_{2}\right) \leq\|\sqrt{L+V} u\|_{2} \leq C_{V}\left(\left\||V|^{\frac{1}{2}} u\right\|_{2}+\|\nabla u\|_{2}\right)
$$

for all $u \in H^{1, V}\left(\mathbb{R}^{n} ; \mathbb{C}^{m}\right)$. Moreover, the constant $C_{V}$ depends on $V$ and $\alpha$ through

$$
C_{V}=\tilde{C}_{V}(\alpha-1)^{-1}\left(1+[V]_{\alpha}^{2}\right),
$$

where $\tilde{C}_{V}$ only depends on $V$ through $\kappa_{A}^{V}$ and is independent of $\alpha$.

Proof The polar decomposition theorem asserts the existence of some $U: \mathbb{R}^{n} \rightarrow$ $\mathcal{L}\left(\mathbb{C}^{m}\right)$, with $U(x)$ unitary for all $x \in \mathbb{R}^{n}$, such that

$$
V(x)=U(x)|V(x)|
$$

for all $x \in \mathbb{R}^{n}$. As $V(x)$ is normal, the matrices $U(x)$ and $|V(x)|$ are well-known to commute. We therefore have the decomposition

$$
V(x)=|V(x)|^{\frac{1}{2}} U(x)|V(x)|^{\frac{1}{2}}
$$

for almost every $x \in \mathbb{R}^{n}$. Set

$$
D:=\nabla: H^{1}\left(\mathbb{R}^{n} ; \mathbb{C}^{m}\right) \subset L^{2}\left(\mathbb{R}^{n} ; \mathbb{C}^{m}\right) \rightarrow L^{2}\left(\mathbb{R}^{n} ; \mathbb{C}^{n} \otimes \mathbb{C}^{m}\right)
$$

and

$$
J:=|V|^{\frac{1}{2}}: D\left(|V|^{\frac{1}{2}}\right) \subset L^{2}\left(\mathbb{R}^{n} ; \mathbb{C}^{m}\right) \rightarrow L^{2}\left(\mathbb{R}^{n} ; \mathbb{C}^{m}\right),
$$

both defined as operators on $L^{2}\left(\mathbb{R}^{n} ; \mathbb{C}^{m}\right)$. Define the perturbation matrices

$$
B_{1}:=I \quad \text { and } \quad B_{2}=\left(\begin{array}{ccc}
I & 0 & 0 \\
0 & U & 0 \\
0 & 0 & A
\end{array}\right)
$$

It is not too difficult to see that the operators $\left\{\Gamma_{0}, B_{1}, B_{2}\right\}$ will satisfy conditions (H1)(H8) and $\left\{\Gamma_{J}, B_{1}, B_{2}\right\}$ will satisfy $(\mathrm{H} 1)-(\mathrm{H} 6)$. Indeed, the only non-trivial condition for both sets of operators is $(\mathrm{H} 2)$ and this follows from the respective Gårding inequalities (1) and (6). It is also clear from the fact that $V \in \mathcal{W}_{\alpha}\left(\mathbb{R}^{n} ; \mathcal{L}\left(\mathbb{C}^{m}\right)\right)$ that $(\mathrm{H} 8 \mathrm{D} \alpha)$ and $(\mathrm{H} 8 \mathrm{~J} \alpha)$ will both be satisfied. This follows from an argument identical to that of Lemma 5.1. The Kato estimate then follows from Corollary 3.2 with constant $\tilde{C}_{V}(\alpha-1)^{-1}\left(1+[V]_{\alpha}^{2}\right)$. It should be noted that (66) was needed so that we would have $L_{B}^{J}=L+V$. 
In analogy to the scalar case, it is quite likely that a similar reverse Hölder type condition will be sufficient to imply the boundedness of the operator $|V|(|V|-\Delta)^{-1}$ for $m>1$. However, as far as the author is aware, this is still an open problem for $m>1$. What is apparent is that the condition that the potential belongs to $L^{\frac{n}{2}}$ will once again be sufficient to imply that it belongs to $\mathcal{W}_{2}$. The following proposition has an identical proof to that of Proposition 5.3.

Proposition 5.4 For $n>4$ and $m \in \mathbb{N}^{*}$,

$$
L^{\frac{n}{2}}\left(\mathbb{R}^{n} ; \mathcal{L}\left(\mathbb{C}^{m}\right)\right) \subset \mathcal{W}_{2}\left(\mathbb{R}^{n} ; \mathcal{L}\left(\mathbb{C}^{m}\right)\right)
$$

\subsection{First Order Potentials}

Let $b: \mathbb{R}^{n} \rightarrow \mathbb{C}^{n}$ be contained in $L_{\text {loc }}^{1}\left(\mathbb{R}^{n} ; \mathbb{C}^{n}\right)$ and $A \in L^{\infty}\left(\mathbb{R}^{n} ; \mathcal{L}\left(\mathbb{C}^{n}\right)\right)$. Suppose that $A$ satisfies the standard Gårding inequality

$$
\operatorname{Re} \int_{\mathbb{R}^{n}}\langle A(x) \nabla u(x), \nabla u(x)\rangle_{\mathbb{C}^{n}} d x \geq \kappa^{A} \cdot\|\nabla u\|_{2}^{2}
$$

for all $u \in H^{1}\left(\mathbb{R}^{n} ; \mathbb{C}\right)$, for some $\kappa^{A}>0$. Consider the accretive sesquilinear form

$$
\mathfrak{h}_{b}^{A}[u, v]:=\langle A \nabla u, \nabla v\rangle_{2}+\langle(\nabla+b) u,(\nabla+b) v\rangle_{2}
$$

defined on the dense subspace $H_{b}^{1}\left(\mathbb{R}^{n}\right):=\left\{u \in H^{1}\left(\mathbb{R}^{n}\right):|b u| \in L^{2}\left(\mathbb{R}^{n}\right)\right\} \subset$ $L^{2}\left(\mathbb{R}^{n}\right)$.

Lemma 5.2 The form $\mathfrak{h}_{b}^{A}$ is both continuous and closed.

Proof Let's first prove that $\mathfrak{h}_{b}^{A}$ is continuous. It must be shown that $\left|\mathfrak{h}_{b}^{A}[u, v]\right| \lesssim$ $\|u\|_{\mathfrak{h}_{b}^{A}}\|v\|_{\mathfrak{h}_{b}^{A}}$ for all $u, v \in H_{b}^{1}$, where $\|u\|_{\mathfrak{h}_{b}^{A}}:=\sqrt{\operatorname{Re}\left(\mathfrak{h}_{b}^{A}[u, u]\right)+\|u\|_{2}^{2}}$. On consecutively applying the boundedness of $A$ and then the accretivity of $A$,

$$
\begin{aligned}
\left|\mathfrak{h}_{b}^{A}[u, v]\right| & =\left|\langle A \nabla u, \nabla v\rangle_{2}+\langle(\nabla+b) u,(\nabla+b) v\rangle_{2}\right| \\
& \lesssim\|\nabla u\|_{2}\|\nabla v\|_{2}+\|(\nabla+b) u\|_{2}\|(\nabla+b) v\|_{2} \\
& \lesssim \sqrt{\operatorname{Re}\langle A \nabla u, \nabla u\rangle_{2}} \sqrt{\operatorname{Re}\langle A \nabla v, \nabla v\rangle_{2}}+\|(\nabla+b) u\|_{2}\|(\nabla+b) v\|_{2} \\
& \lesssim\|u\|_{\mathfrak{h}_{b}^{A}}\|v\|_{\mathfrak{h}_{b}^{A}} .
\end{aligned}
$$

Let's now prove that $\mathfrak{h}_{b}^{A}$ is closed. That is, it must be proved that $H_{b}^{1}\left(\mathbb{R}^{n}\right)$ is complete under the norm $\|\cdot\|_{\mathfrak{h}_{b}^{A}}$. From the boundedness of $A$,

$$
\begin{aligned}
\|u\|_{\mathfrak{h}_{b}^{A}} & =\sqrt{\operatorname{Re}\left(\mathfrak{h}_{b}^{A}[u, u]\right)+\|u\|_{2}^{2}} \\
& =\sqrt{\operatorname{Re}\left(\langle A \nabla u, \nabla u\rangle_{2}\right)+\|(\nabla+b) u\|_{2}^{2}+\|u\|_{2}^{2}}
\end{aligned}
$$




$$
\begin{aligned}
& \lesssim \sqrt{\|\nabla u\|_{2}^{2}+\|b u\|_{2}^{2}+\|u\|_{2}^{2}} \\
& \lesssim\|u\|_{H_{b}^{1}}
\end{aligned}
$$

where $\|u\|_{H_{b}^{1}}:=\|u\|_{2}+\|\nabla u\|_{2}+\|b u\|_{2}$. Conversely, the Gårding inequality for $A$ implies that

$$
\begin{aligned}
\|u\|_{\mathfrak{h}_{b}^{A}} & =\sqrt{\operatorname{Re}\left(\langle A \nabla u, \nabla u\rangle_{2}\right)+\|(\nabla+b) u\|_{2}^{2}+\|u\|_{2}^{2}} \\
& \geq\|\nabla u\|_{2}+\|(\nabla+b) u\|_{2}+\|u\|_{2} \\
& \geq\|\nabla u\|_{2}+\frac{1}{2}\|(\nabla+b) u\|_{2}+\|u\|_{2} \\
& \geq\|\nabla u\|_{2}+\frac{1}{2}\|b u\|_{2}-\frac{1}{2}\|\nabla u\|_{2}+\|u\|_{2} \\
& \simeq\|u\|_{H_{b}^{1}} .
\end{aligned}
$$

This shows that the norm $\|\cdot\|_{\mathfrak{h}_{b}^{A}}$ is equivalent to $\|\cdot\|_{H_{b}^{1}}$ on $H_{b}^{1}$. Since $H_{b}^{1}$ is known to be complete under the norm $\|\cdot\|_{H_{b}^{1}}$ it then follows that it must also be complete under $\|\cdot\|_{\mathfrak{h}_{b}^{A}}$.

The previous lemma implies, in particular, that there exists a maximal accretive operator $\mathcal{L}_{b}^{A}$ associated with this form (c.f. [19, Sect. 1.2]). This operator will be denoted by

$$
\mathcal{L}_{b}^{A}=(\nabla+b)^{*}(\nabla+b)-\operatorname{div}(A \nabla)
$$

Define the Hilbert space to be

$$
\mathcal{H}:=L^{2}\left(\mathbb{R}^{n}\right) \oplus L^{2}\left(\mathbb{R}^{n} ; \mathbb{C}^{n}\right) \oplus L^{2}\left(\mathbb{R}^{n} ; \mathbb{C}^{n}\right)
$$

Then set

$J:=\nabla+b: L^{2}\left(\mathbb{R}^{n} ; \mathbb{C}\right) \rightarrow L^{2}\left(\mathbb{R}^{n} ; \mathbb{C}^{n}\right)$ and $D:=\nabla: L^{2}\left(\mathbb{R}^{n} ; \mathbb{C}\right) \rightarrow L^{2}\left(\mathbb{R}^{n} ; \mathbb{C}^{n}\right)$

Also, let $B_{1}=I$ as usual and

$$
B_{2}=\left(\begin{array}{lll}
I & 0 & 0 \\
0 & I & 0 \\
0 & 0 & A
\end{array}\right) .
$$

Then the operator $L_{B}^{J}$ as in Corollary 3.2 is given by $L_{B}^{J}=\mathcal{L}_{b}^{A}$. Since $A$ satisfies the standard Gårding inequality it follows that $\left\{\Gamma_{0}, B_{1}, B_{2}\right\}$ and $\left\{\Gamma_{J}, B_{1}, B_{2}\right\}$ will both satisfy (H2). This in turn implies that $\left\{\Gamma_{0}, B_{1}, B_{2}\right\}$ satisfies $(\mathrm{H} 1)-(\mathrm{H} 8)$ and $\left\{\Gamma_{J}, B_{1}, B_{2}\right\}$ satisfies (H1)-(H6). The non-homogeneous framework, in the form of Corollary 3.2, applied to these operators then produces the following theorem. 
Theorem 5.3 Suppose that $D\left((\nabla+b)^{*}(\nabla+b)-\Delta\right) \subset D(-\Delta) \cap D\left((\nabla+b)^{*}(\nabla+b)\right)$ and there exists some $c_{b}>0$ such that

$$
\|\Delta u\|_{2} \leq c_{b}\left\|\left[(\nabla+b)^{*}(\nabla+b)-\Delta\right] u\right\|_{2}
$$

for all $u \in D\left((\nabla+b)^{*}(\nabla+b)-\Delta\right)$. Then there exists some constant $c>0$, independent of $b$, for which

$$
\begin{aligned}
\left(c \cdot\left(1+c_{b}\right)\right)^{-2}\left(\|(\nabla+b) u\|_{2}+\|\nabla u\|_{2}\right) & \leq\left\|\sqrt{\mathcal{L}_{b}^{A}} u\right\|_{2} \\
& \leq\left(c \cdot\left(1+c_{b}\right)\right)^{2}\left(\|(\nabla+b) u\|_{2}+\|\nabla u\|_{2}\right)
\end{aligned}
$$

for all $u \in H_{b}^{1}\left(\mathbb{R}^{n}\right)$.

To see that the above theorem is true, simply note that (67) implies both (H8D $\alpha)$ and ( $\mathrm{H} 8 \mathrm{~J} \alpha)$ for $\alpha=2$ in this context. The independence of the constant $c$ from $b$ follows from the fact that (H2) is satisfied by $\left\{\Gamma_{J}, B_{1}, B_{2}\right\}$ with constant independent of $b$.

\section{Literature Comparison}

It is important to note that this is not the first time that Kato-type estimates have been studied for non-homogeneous operators. We will now take some time to outline how our article differs in techniques and results from each of these previous forays.

Recently, in [11] and [12], Gesztesy, Hofmann and Nichols studied the domains of square root operators using techniques distinct from those developed in [6]. The article [11] considers potentials in the class $L^{p}+L^{\infty}$ but is not directly relevant since it considers bounded domains. On the other hand, [12] does not impose a boundedness assumption on the domain and considers the potential class $L^{\frac{n}{2}}+L^{\infty}$. There is already an immediate comparison with our potential class since it was shown in Proposition 5.3 that $L^{\frac{n}{2}} \subset \mathcal{W}$ in dimension $n>4$. It is not immediately clear whether $L^{\infty}$ is contained within our class.

Axelsson, Keith and McIntosh themselves considered non-homogeneous operators on Lipschitz domains with mixed boundary conditions in [5]. The potentials that they considered were, however, bounded both from above and below and thus contained in $\mathrm{RH}_{2} \subset \mathcal{W}$. In [8] and [9], Egert, Haller-Dintelmann and Tolksdorf generalised this to certain non-smooth domains.

The articles [5], [8] and [9] are built upon the original AKM framework, similar to this one. A key step in the original proof of the AKM framework is the proof of the estimate

$$
\int_{0}^{\infty}\left\|\left(A_{t}-P_{t}\right) u\right\|^{2} \frac{d t}{t} \lesssim\|u\|^{2} .
$$

This estimate allows for the $A_{t}$ and $P_{t}$ operators to be freely interchanged at several stages in the proof granting use of some of the more enviable properties of the $A_{t}$ 
operator. As has been demonstrated in this article through the diagonalisation theorem, Theorem 4.1, (68) will not hold for a general potential. The articles [5,8,9], circumvent this problem by imposing boundedness of the potential from above and below. The boundedness of the potential from above and below allows one to absorb the potential into the multiplicative perturbation $B_{2}$ so that you are instead considering the operators

$$
\Gamma_{1}=\left(\begin{array}{ccc}
0 & 0 & 0 \\
1 & 0 & 0 \\
\nabla & 0 & 0
\end{array}\right), \quad B_{1}=I \quad \text { and } \quad B_{2}=\left(\begin{array}{ccc}
I & 0 & 0 \\
0 & V & 0 \\
0 & 0 & A
\end{array}\right)
$$

For this set of operators, the large time-scale estimate

$$
\int_{1}^{\infty}\left\|\Theta_{t}^{B, 1} P_{t}^{1} u\right\|^{2} \frac{d t}{t} \lesssim \int_{1}^{\infty}\left\|P_{t}^{1} u\right\|^{2} \frac{d t}{t} \lesssim \int_{1}^{\infty}\left\|t \Pi_{1} P_{t}^{1} u\right\|^{2} \frac{d t}{t} \lesssim\|u\|^{2}
$$

for $u \in R\left(\Gamma_{1}\right)$ follows almost trivially. Then one only requires a small time-scale version of (68) to hold, namely

$$
\int_{0}^{1}\left\|\left(A_{t}-P_{t}^{1}\right) u\right\|^{2} \frac{d t}{t} \lesssim\|u\|^{2}
$$

for all $u \in R\left(\Gamma_{1}\right)$. Such an estimate is then proved to be true.

This is a crude explanation as to why the techniques developed in [5] cannot be directly applied for a general potential that is not bounded both from above and below. There are similar obstructions, for example in the selection of test functions in the Carleson measure proof. However, these also disappear when the potential is bounded both from above and below.

In this paper, our method has been to instead treat the potential as part of the unperturbed operator and then to subsequently exploit the algebraic structure of the operators $\Gamma_{|V|^{\frac{1}{2}}}, B_{1}$ and $B_{2}$. This exploitation has allowed us to conclude that the estimate (68) will at least hold on the third component which, it turns out, is all that is required. Similar obstructions in the proof of the main square function estimate also vanish when considered component-wise. As a consequence of this three-bythree mindset we have been able to obtain square function estimates for potentials that aren't necessarily bounded from above or below and, moreover, are not contained in $L^{p}\left(\mathbb{R}^{n}\right)$ for any $1 \leq p \leq \infty$.

Acknowledgements This paper is part of my $\mathrm{PhD}$ thesis undertaken at the Australian National University. I am very thankful to my supervisor Pierre Portal for his numerous suggestions and corrections and for the encouragement that made this article possible. I am indebted to El Maati Ouhabaz for the discussions surrounding this project and for years ago suggesting to Pierre that Kato estimates for Schrödinger-type operators would be of particular interest to the community, thus initiating this line of research. I am also grateful to my second supervisor Adam Sikora for his sage advice, in particular with regards to Proposition 5.3. A part of this paper was written while visiting him at Macquarie University. While this paper was in its final stages of preparation, it was found that Andrew Morris and Andrew Turner from the University of Birmingham were also working on the Kato problem with potential on $\mathbb{R}^{n}$. After meeting them and discussing their research, it appears that the two approaches differ in their assumptions, their results and, more substantially, their proofs. I would like to thank Moritz Egert for spotting an error in a previous version 
of this article and for his comments on Proposition 5.2. Finally, I would like to thank the anonymous referees of previous versions of this article for providing such detailed and thoughtful critiques. Reflecting on the comments provided in their reports led me to several significant improvements.

Open Access This article is licensed under a Creative Commons Attribution 4.0 International License, which permits use, sharing, adaptation, distribution and reproduction in any medium or format, as long as you give appropriate credit to the original author(s) and the source, provide a link to the Creative Commons licence, and indicate if changes were made. The images or other third party material in this article are included in the article's Creative Commons licence, unless indicated otherwise in a credit line to the material. If material is not included in the article's Creative Commons licence and your intended use is not permitted by statutory regulation or exceeds the permitted use, you will need to obtain permission directly from the copyright holder. To view a copy of this licence, visit http://creativecommons.org/licenses/by/4.0/.

\section{References}

1. Albrecht, D., Duong, X., McIntosh, A.: Operator theory and harmonic analysis. In: Instructional Workshop on Analysis and Geometry, Part III (Canberra, 1995). Proc. Centre Math. Appl. Austral. Nat. Univ 34, 77-136 (1996)

2. Auscher, P., Ben Ali, B.: Maximal inequalities and Riesz transform estimates on $L^{p}$ spaces for Schrödinger operators with nonnegative potentials. Ann. Inst. Fourier (Grenoble) 57, 1975-2013 (2007)

3. Auscher, P., Hofmann, S., McIntosh, A., Tchamitchian, P.: The Kato square root problem for higher order elliptic operators and systems on $\mathbb{R}^{n}$. J. Evol. Equ. 1, 361-385 (2001)

4. Auscher, P., Hofmann, S., Lacey, M., McIntosh, A., Tchamitchian, P.: The solution of the Kato square root problem for second order elliptic operators on $\mathbb{R}^{n}$. Ann. Math. (2) 156, 633-654 (2002)

5. Axelsson, A., Keith, S., McIntosh, A.: The Kato square root problem for mixed boundary value problems. J. Lond. Math. Soc. (2) 74, 113-130 (2006)

6. Axelsson, A., Keith, S., McIntosh, A.: Quadratic estimates and functional calculi of perturbed Dirac operators. Invent. Math. 163, 455-497 (2006)

7. Cowling, M., Doust, I., McIntosh, A., Yagi, A.: Banach space operators with a bounded $H^{\infty}$ functional calculus. J. Austral. Math. Soc. Ser. A 60, 51-89 (1996)

8. Egert, M., Haller-Dintelmann, R., Tolksdorf, P.: The Kato square root problem for mixed boundary conditions. J. Funct. Anal. 267, 1419-1461 (2014)

9. Egert, M., Haller-Dintelmann, R., Tolksdorf, P.: The Kato square root problem follows from an extrapolation property of the Laplacian. Publ. Mat. 60, 451-483 (2016)

10. Frey, D., McIntosh, A., Portal, P.: Conical square function estimates and functional calculi for perturbed Hodge-Dirac operators in $L^{p}$. J. Anal. Math. 134, 399-453 (2018)

11. Gesztesy, F., Hofmann, S., Nichols, R.: Stability of square root domains associated with elliptic systems of PDEs on nonsmooth domains. J. Differ. Equ. 258, 1749-1764 (2015)

12. Gesztesy, F., Hofmann, S., Nichols, R.: On stability of square root domains for non-self-adjoint operators under additive perturbations. Mathematika 62, 111-182 (2016)

13. Grafakos, L.: Modern Fourier Analysis, vol. 250. Springer, New York (2009)

14. Haase, M.: The Functional Calculus for Sectorial Operators. Birkhaüser Verlag, Basel (2006)

15. Hofmann, S., Lacey, M., McIntosh, A.: The solution of the Kato problem for divergence form elliptic operators with Gaussian heat kernel bounds. Ann. Math. (2) 156, 623-631 (2002)

16. Hytönen, T., Van Neerven, J., Veraar, M., Weis, L.: Analysis in Banach Spaces-Volume II Probabilistic Methods and Operator Theory, vol. 2. Springer, New York (2017)

17. Kato, T.: Perturbation Theory for Linear Operators, vol. 132. Springer, New York (1980)

18. Morris, A.J.: The Kato square root problem on submanifolds. J. Lond. Math. Soc. (2) 86, 879-910 (2012)

19. Ouhabaz, E.-M.: Analysis of Heat Equations on Domains (LMS-31). Princeton University Press, Princeton (2005)

20. Semenov, Y.A.: Schrödinger operators with $L_{l o c}^{p}$-potentials. Commun. Math. Phys. 53, 277-284 (1977)

21. Shen, Z.: $L^{p}$ estimates for Schrödinger operators with certain potentials. Ann. Inst. Fourier (Grenoble) 45, 513-546 (1995) 
22. Stein, E.M.: Harmonic Analysis: Real-Variable Methods, Orthogonality, and Oscillatory Integrals, vol. 43. Princeton University Press, Princeton (1993)

Publisher's Note Springer Nature remains neutral with regard to jurisdictional claims in published maps and institutional affiliations. 